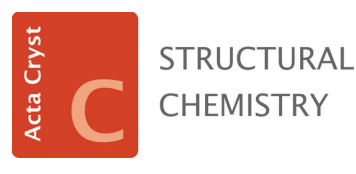

ISSN 2053-2296

Received 4 August 2017

Accepted 16 October 2017

Edited by B. D. Santarsiero, University of Illinois at Chicago, USA

Keywords: 2D NMR; positional disorder; complementary analytical techniques; crystal structure.

CCDC reference: 1580102

Supporting information: this article has supporting information at journals.iucr.org/C

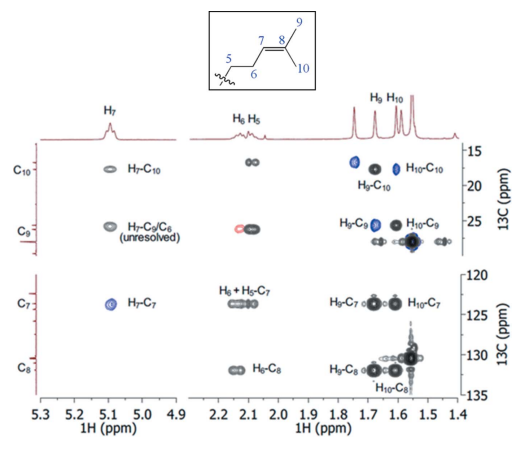

C 2017 International Union of Crystallography

\section{Deciphering composition and connectivity of a natural product with the assistance of MS and 2D NMR}

\author{
Anastasiya I. Vinokur, ${ }^{a}$ Paul B. White, ${ }^{\mathrm{b}}$ Maurice Tagatsing Fotsing, ${ }^{\mathrm{c}}$ Charmaine \\ Arderne, ${ }^{\mathrm{d}}$ Derek Tantoh Ndinteh, ${ }^{\mathrm{e}}$ Martha M. Vestling ${ }^{\mathrm{a}}$ and Ilia A. Guzei ${ }^{\mathrm{a}, \mathrm{d} *}$
}

\author{
${ }^{a}$ Department of Chemistry, University of Wisconsin-Madison, 1101 University Ave, Madison, WI 53706, USA, ' Institute \\ for Molecules and Materials, Radboud University, Nijmegen, Heyendaalseweg 135, 6525 AJ, The Netherlands, \\ ${ }^{\mathbf{c}}$ Department of Organic Chemistry, University of Yaounde I, PO Box 812, Yaounde, Cameroon, 'Department of \\ Chemistry, University of Johannesburg, PO Box 524, Auckland Park, Johannesburg 2006, South Africa, and ${ }^{\mathbf{e} D e p a r t m e n t}$ \\ of Applied Chemistry, University of Johannesburg, PO Box 17011, Doornfontein, Johannesburg 2028, South Africa. \\ *Correspondence e-mail: iguzei@chem.wisc.edu
}

A complementary application of three analytical techniques, viz. multidimensional nuclear magnetic resonance spectroscopy (NMR), mass spectrometry (MS), and single-crystal X-ray diffractometry was required to identify and refine two natural products isolated from Millettia versicolor and solvent of crystallization. The two compounds, namely 3-(2H-1,3-benzodioxol-5-yl)-6-methoxy8,8-dimethyl-4H,8H-pyrano[2,3- $h]$ chromen-4-one, or durmillone, (I), and (2E)1-(4-\{[(2E)-3,7-dimethylocta-2,6-dien-1-yl]oxy\}-2-hydroxyphenyl)-3-(4-hydroxyphenyl)prop-2-en-1-one, (II), could not be separated by routine column chromatography and cocrystallized in a 2:1 ratio with 0.13 molecules of ethanol solvent. Compound (II) and ethanol could not be initially identified by singlecrystal X-ray analysis due to complex disorder in the aliphatic chain region of (II). Mass spectrometry ensured that (II) represented only one species disordered over several positions in the solid state, rather than several species cohabitating on the same crystallographic site. The atomic identification and connectivity in (II) were established by several 2D (two-dimensional) NMR techniques, which in turn relied on a knowledge of its exact mass. The derived connectivity was then used in the single-crystal analysis to model the disorder of the aliphatic chain in (II) over three positions and allowed identification of a partially occupied ethanol solvent molecule that was disordered over an inversion center. The disordered moieties were refined with restraints and constraints.

\section{Introduction}

Flavonoids and isoflavonoids are recognized mostly as coming from the plant family Fabaceae (Veitch, 2007, 2009, 2013). Compounds isolated from the roots, leaves, and stem bark of Millettia versicolor (from the Fabaceae family) are of interest because the extracts from this plant have been shown to possess anti-inflammatory properties (Fotsing et al., 2003).

The goals of the current study were to solvent extract different parts of the botanically isolated plants with organic solvents, and chromatographically isolate and characterize the secondary metabolites responsible for the observed bioactivity. Unequivocal identification of the constituents of the extract posed significant challenges due to the difficulty of separation of the components. In the present work, the two compounds in question could not be separated by the available laboratory methods, including column chromatography and preparative thin-layer chromatography (TLC). Frequently, we obtain novel compounds for which there may be 
no standards, thus the only way of elucidating structures is by using complementary spectroscopic and X-ray diffraction techniques.

This applies both to natural products and to their synthetically prepared congeners. In the fortunate instances when crystals of the product(s) are available, single-crystal X-ray diffraction (SCXRD) is used to authenticate the structures and possibly establish their absolute configuration. Recent examples of natural compounds whose structures were first elucidated by spectroscopic techniques and subsequently confirmed by SCXRD, include bioactive isoflavonoid phytochemicals extracted from Garcinia nervosa (Parveen et al., 2017), novel isoflavonoids from Cassia siamea (Parveen et al., 2016), 12 new flavan derivatives from Populus balsamifera (Simard et al., 2014), a number of natural products with extensive antimicrobial properties (Saleem et al., 2010), a novel iridoid compound from Torricellia angulate var. intermedia (Liang et al., 2009), bioactive diterpenes from Croton steenkampianus (Adelekan et al., 2008), extracts of diterpenoid glycosides from Gleichenia quadripartite (Socolsky et al., 2007), flavonoids from the roots of Dioclea grandiflora (Lemos et al., 2006), a novel diterpenoid from Ballota limbeta (Riaz et al., 2004), and novel isoflavones from Ceiba pentandra (Ngounou et al., 2000).<smiles>COc1cc2c(=O)c(-c3ccc4c(c3)OCO4)coc2c2c1OC(C)(C)C=C2</smiles>

(I)<smiles>CCO[GaH]O</smiles>
Scheme 1

Solution 1D and 2D (one- and two-dimensional) NMR techniques have become common for determining chemically specific detailed structural information about organic and organometallic compounds (Claridge, 2016; Friebolin, 2010; Berger \& Braun, 2004). In our case, the interpretation of the NMR spectrum of the yet unknown (II) was somewhat complicated by the presence of a second species, i.e. (I), but it was possible to obtain local structural information about (II) and subsequently build a multiconformer crystallographic model.

We report herein structural studies of two natural products isolated from the stem bark of Millettia versicolor, namely durmillone, (I), and (2E)-1-(4-\{[(2E)-3,7-dimethylocta-2,6dien-1-yl]oxy\}-2-hydroxyphenyl)-3-(4-hydroxyphenyl)prop-2en-1-one, (II), with a detailed interpretation of the 2D NMR and MS spectra, and a description of the crystallographic
Table 1

Full assignment of unknown species (X) that was later established as (II) (see Fig. 1).

\begin{tabular}{llll}
\hline Label & ${ }^{1} \mathrm{H}(\mathrm{ppm})$ & ${ }^{13} \mathrm{C}(\mathrm{ppm})$ & Predicted ${ }^{13} \mathrm{C}(\mathrm{ppm})$ \\
\hline 1 & 4.59 & 67.3 & 65.4 \\
2 & 5.48 & 118.5 & 119.6 \\
3 & - & 142.3 & 142.8 \\
4 & 1.75 & 16.8 & 16.7 \\
5 & 2.09 & 39.5 & 39.6 \\
6 & 2.13 & 26.3 & 26.3 \\
7 & 5.09 & 123.6 & 123.8 \\
8 & - & 132.0 & 132.2 \\
9 & 1.68 & 25.8 & 25.8 \\
10 & 1.61 & 17.7 & 17.7 \\
11 & - & 165.6 & 165.3 \\
12 & 6.48 & 101.7 & 101.6 \\
13 & $13.55(\mathrm{OH})$ & 166.6 & 166.6 \\
14 & - & 114.0 & 114.0 \\
15 & 7.82 & 131.1 & 131.1 \\
16 & 6.49 & 108.2 & 111.0 \\
17 & - & 191.8 & 191.7 \\
18 & 7.45 & 117.8 & 117.5 \\
19 & 7.84 & 144.2 & 145.9 \\
20 & - & 127.4 & 127.6 \\
21 & 7.55 & 130.5 & 130.5 \\
22 & 6.88 & 116.0 & 116.1 \\
23 & - & 158.4 & 158.4 \\
\hline
\end{tabular}

refinement. These compounds form a 2(I):(II) cocrystal, with 0.13 molecules of solvent ethanol (see Scheme 1).

\section{Experimental}

\subsection{Synthesis and crystallization}

The Millettia versicolor stem bark was collected in Cameroon and the details recorded at Herbier National du Cameroun (HNC) under voucher specimen 45582 HNC. The stem bark was air dried, finely ground, and stored. About $500 \mathrm{~g}$ of the dried stem bark were weighed and soaked at room temperature and pressure in 1.6 l of a 1:1 $(\mathrm{v} / \mathrm{v})$ mixture of methylene dichloride (DCM) and $\mathrm{MeOH}$ in a closed vessel for $72 \mathrm{~h}$. After $72 \mathrm{~h}$, the plant material was filtered off using a normal Whatman filter paper under gravity. These extractions

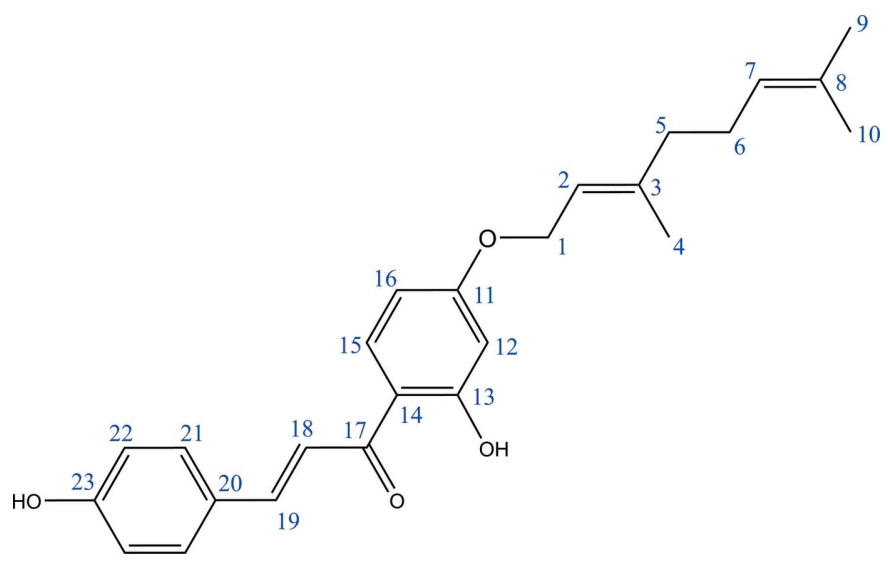

Figure 1

The atom-labeling scheme for the NMR investigation of (II) (see Table 1). 
Table 2

Ions in positive ion mode; $M=(\mathrm{I})$.

\begin{tabular}{lcrl}
\hline Ion & Calculated & Measured & Difference \\
\hline$[M+\mathrm{H}]^{+}$ & 379.1176 & 379.1170 & 0.0006 \\
{$[2 M+\mathrm{Na}]^{+}$} & 779.2099 & 779.2089 & 0.0010 \\
{$[3 M+\mathrm{Na}]^{+}$} & 1157.3202 & 1157.3207 & 0.0005 \\
\hline
\end{tabular}

Table 3

Ions in negative ion mode; $M=(\mathrm{II})$.

\begin{tabular}{llll}
\hline Ion & Calculated & Measured & Difference \\
\hline$[M-\mathrm{H}]^{-}$ & 391.2279 & 391.1918 & 0.0361 \\
{$\left[M-\mathrm{C}_{10} \mathrm{H}_{17}\right]^{-}$} & 254.0948 & 254.0587 & 0.0361 \\
{$\left[M-\mathrm{C}_{9} \mathrm{H}_{8} \mathrm{O}_{2}\right]^{-}$} & 148.0530 & 148.0168 & 0.0362 \\
\hline
\end{tabular}

were repeated seven times and the combined solvents were removed using a rotary evaporator (Buchi, Switzerland) under reduced pressure. The resulting extract was then stored at $277 \mathrm{~K}$ until it was required for use.

The extract was chromatographed over silica gel using gradient solvent elution with hexane, DCM, and methanol. The compound, which was code-named MTF 20, crystallized out of the hexane/DCM eluent as pale-yellow crystals, which were filtered off and stored in an airtight container for structural characterization and biological evaluation.

\subsection{NMR studies}

All NMR spectra were collected on a Bruker $600 \mathrm{MHz}$ AvanceHD spectrometer equipped with a TCI-F cryoprobe at $298 \mathrm{~K}$ with a ${ }^{1} \mathrm{H}$ frequency of $599.77 \mathrm{MHz}$ and a ${ }^{13} \mathrm{C}$ frequency of $150.83 \mathrm{MHz}$. The 2D COSYDQF (correlation spectroscopy double quantum filter) spectrum was acquired with a $8620.7 \mathrm{~Hz}$ spectral width in both dimensions using $2048 \times 512$ points and processed using $8192 \times 1024$ points, 2 scans per increment, and a relaxation delay of $2 \mathrm{~s}$. Multiplicity-edited ${ }^{1} \mathrm{H}-{ }^{13} \mathrm{C}$ HSQC (heteronuclear single quantum coherence) spectra were acquired using a $9014.4 \mathrm{~Hz}$ spectral width in F2 and a $27174 \mathrm{~Hz}$ spectral width in F1, using $1024 \times 512$ points, and processed to $1024 \times 1024$ points, 2 scans per increment, a relaxation delay of $2 \mathrm{~s}$, and 1-bond $J_{\mathrm{CH}}=145 \mathrm{~Hz} \cdot{ }^{1} \mathrm{H}-{ }^{13} \mathrm{C}$ HMBC (heteronuclear multiple bond correlation) spectra were acquired using a $9014.4 \mathrm{~Hz}$ spectral width in F2 and a $31446.5 \mathrm{~Hz}$ spectral width in $\mathrm{F} 1$, using $2048 \times 512$ points, and processed to $2048 \times 2048$ points, 8 scans per increment, a relaxation delay of $2 \mathrm{~s}$, and long-range $J_{\mathrm{CH}}=8 \mathrm{~Hz}$. 1D NOESY

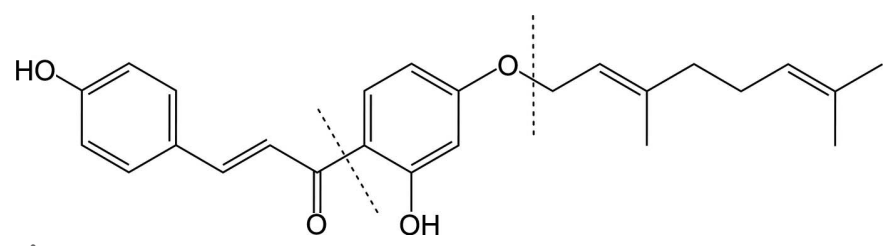

Figure 2

Fragmentation pattern of (II) under negative ion electrospray ionization conditions.
Table 4

Experimental details.

\begin{tabular}{ll}
\hline Crystal data & \\
Chemical formula & $2 \mathrm{C}_{22} \mathrm{H}_{18} \mathrm{O}_{6} \cdot \mathrm{C}_{25} \mathrm{H}_{28} \mathrm{O}_{4} \cdot 0.13 \mathrm{C}_{2} \mathrm{H}_{6} \mathrm{O}$ \\
$M_{\mathrm{r}}$ & 1155.42 \\
Crystal system, space group & Triclinic, $P \overline{1}$ \\
Temperature $(\mathrm{K})$ & 100 \\
$a, b, c(\AA)$ & $12.519(3), 12.594(3), 19.975(4)$ \\
$\alpha, \beta, \gamma\left(^{\circ}\right)$ & $80.12(3), 89.69(3), 68.02(3)$ \\
$V\left(\AA^{3}\right)$ & $2871.3(12)$ \\
$Z$ & 2 \\
Radiation type & Mo $K \alpha$ \\
$\mu\left(\mathrm{mm}^{-1}\right)$ & 0.10 \\
Crystal size (mm) & $0.11 \times 0.06 \times 0.02$ \\
& \\
Data collection & Bruker APEXII Quazar \\
Diffractometer & Multi-scan $(S A D A B S ;$ Krause $e t$ \\
Absorption correction & $a l ., 2015)$ \\
& $0.679,0.745$ \\
$T_{\text {min }}, T_{\text {max }}$ & $42663,11806,7371$ \\
No. of measured, independent and & \\
$\quad$ observed $[I>2 \sigma(I)]$ reflections & 0.054 \\
$R_{\text {int }}$ & 0.627 \\
$(\text { sin } \theta / \lambda)_{\text {max }}\left(\AA^{-1}\right)$ & \\
Refinement & \\
$R\left[F^{2}>2 \sigma\left(F^{2}\right)\right], w R\left(F^{2}\right), S$ & $0.057,0.165,1.03$ \\
No. of reflections & 11806 \\
No. of parameters & 871 \\
No. of restraints & 193 \\
$\mathrm{H}$-atom treatment & $\mathrm{H}$-atom parameters constrained \\
$\Delta \rho_{\text {max }}, \Delta \rho_{\text {min }}\left(\mathrm{e} \AA^{-3}\right)$ & $0.47,-0.55$ \\
\hline &
\end{tabular}

Computer programs: APEX3 (Bruker, 2016), SAINT-Plus (Bruker, 2016), SHELXT (Sheldrick, 2015a), SHELXL2016 (Sheldrick, 2015b) and OLEX2 (Dolomanov et al., 2009).

(nuclear overhauser effect spectroscopy) spectra were collected with $500 \mathrm{~ms}$ of mixing time, 32 scans, and a relaxation delay of $3 \mathrm{~s}$ (Table 1 and Fig. 1).

\subsection{Mass spectral analysis}

A portion of $1 \mathrm{mg}$ of the crystalline material was dissolved in $300 \mu \mathrm{l}$ of $10 \mathrm{mM} \mathrm{NH}_{4} \mathrm{OAc} / \mathrm{CH}_{3} \mathrm{CN}$ and subjected to both positive ion $(1 \mu \mathrm{l})$ and negative ion $(1 \mu \mathrm{l})$ electrospray ionization on a Thermo Scientific Q Exactive Plus ${ }^{\mathrm{TM}}$ mass spectrometer. The positive ion spectrum was dominated by three related ions corresponding to compound (I) that could be assigned to $[M+\mathrm{H}]^{+},[2 M+\mathrm{Na}]^{+}$, and $[3 M+\mathrm{Na}]^{+}$(Table 2). The distribution is typical for electrospray ionization from a concentrated solution of analyte. The presence of a phenol predicts an ion of (II) in a negative ion electrospray ionization. The negative ion mass spectrum had one intense ion at $\mathrm{m} / \mathrm{z}$ 391.1918. Its MSMS (tandem) spectrum showed two major losses to give ions at $m / z 254.0587$ and 148.0168 (Table 3 and Fig. 2).

\subsection{Refinement}

Crystal data, data collection and structure refinement details are summarized in Table 4. The single-crystal X-ray diffraction data were acquired in a routine fashion (Bruker, 2012, 2016). The structure was solved and refined by standard techniques (Dolomanov et al., 2009; Krause et al., 2015; Sheldrick, 2015a,b). 


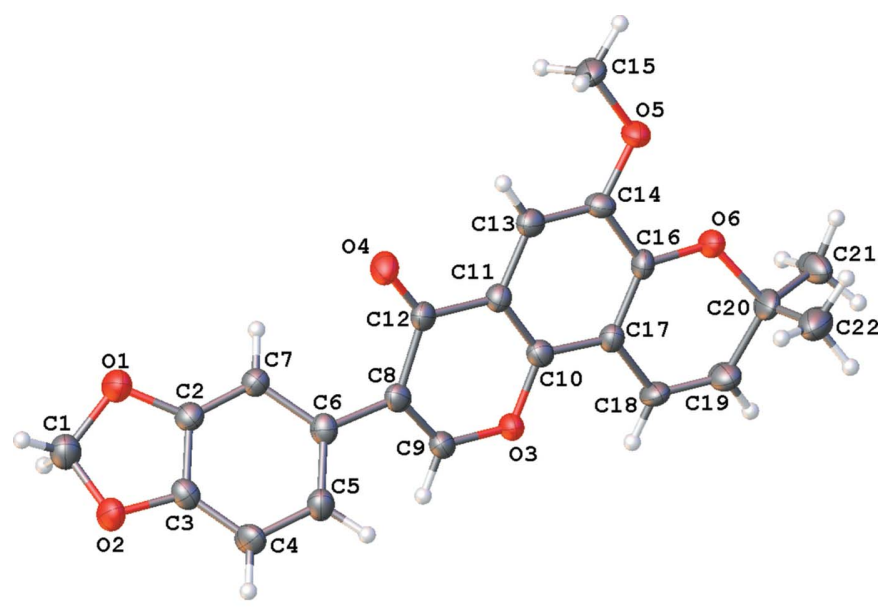

(a)

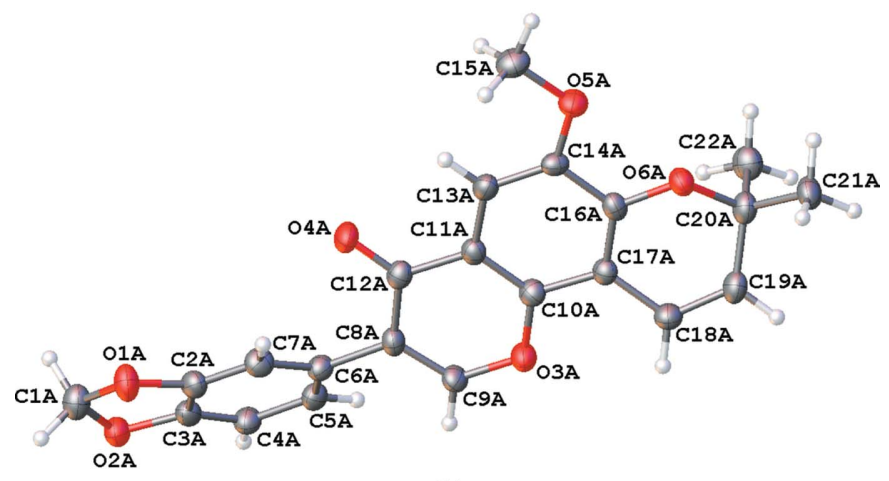

(b)

Figure 3

Molecular drawings of the two independent molecules of (I), showing (a) molecule $(\mathrm{I} a)$ and $(b)$ molecule $(\mathrm{I} b)$, drawn with $50 \%$ probability displacement ellipsoids.

There are four symmetry-independent molecules in the asymmetric unit, i.e. two molecules of (I), one molecule of (II), and 0.13 molecules of ethanol solvent. Both molecules of compound (I) are ordered. Molecule (II) exhibited extensive positional disorder in the chain at atom $\mathrm{C} 40$. Atoms $\mathrm{C} 42-\mathrm{C} 47$ were modelled as disordered over three positions in a 49.7 (3):27.0 (2):23.3 (3) ratio. All 1-2 and 1 $\cdots 3$ interatomic distances in all three of the disordered components were restrained to be the same. The following additional restraints and constraints were applied to the structure to achieve a computationally stable refinement. The $\mathrm{C} 39-\mathrm{C} 40$ and $\mathrm{C} 44-\mathrm{C} 45$ bond lengths were restrained to be similar, as were

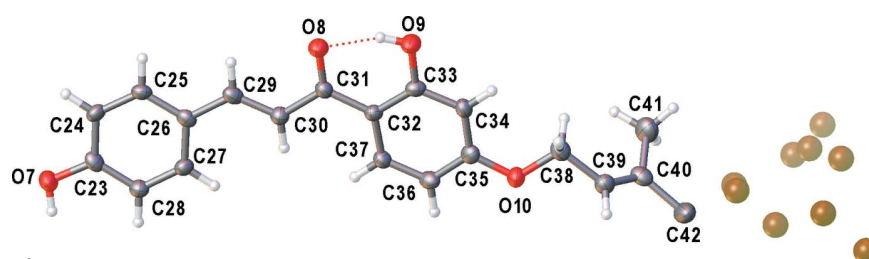

Figure 4

A molecular drawing of $(\mathrm{X})$, shown with the known labeled part of the molecule and the unknown portion of the structure represented by peaks of electron density. The color intensity of the golden spheres is proportional to the peak heights. The displacement ellipsoids are drawn at the $50 \%$ probability level.
$\mathrm{C} 43-\mathrm{C} 44$ and $\mathrm{C} 42-\mathrm{C} 43$. Atomic coordinates, as well as anisotropic displacement parameters, for atoms $\mathrm{C} 42 A$ and $C 42 B$ were constrained to be identical. The anisotropic displacement parameters for atoms $\mathrm{C} 44 B, \mathrm{C} 45 B$, and $\mathrm{C} 46 B$ were constrained to be the same, as were those for atoms C39, $\mathrm{C} 43 A$, and $\mathrm{C} 43 B$.

The ethanol solvent molecule is disordered over a crystallographic inversion center with a partial occupancy of $13.5(2) \%$. This molecule was refined with an idealized geometry (Guzei, 2014).

\section{Results and discussion}

The plant extract under investigation was isolated by thinlayer chromatography (TLC) and subsequent column chromatography $(\mathrm{CC})$ in order to obtain a pure product. However, the product contained two components that could not be separated despite numerous $\mathrm{CC}$ runs executed under various conditions. Separatory CC was the only method utilized as a means of separating the two isolated flavonoids. Reversed phase chromatography (C18) has not been tried, as only normal phase (silica gel) was available. To separate the two compounds, a number of different HPLC (high performance liquid chromatography) or UHPLC (ultra-high performance liquid chromatography) column packings and gradients will need to be tried.

The typical FT-IR and ${ }^{1} \mathrm{H}$ and ${ }^{13} \mathrm{C}$ NMR experiments did not reveal the presence of a known compound; thus SCXRD was considered an ideal technique that could both identify the compound and possibly establish its absolute configuration.

A routine crystallographic characterization revealed that the extract crystallized in a centrosymmetric space group and therefore would be a racemic mixture if asymmetric $\mathrm{C}$ atoms were present. The asymmetric unit contained three symmetryindependent molecules with different compositions and possibly a solvent of crystallization. Thus, the components of the extract could not be resolved by crystallization. Two ordered molecules had identical compositions, hereafter (I $a$ ) and (Ib) (Figs. $3 a$ and $3 b$ ) [PubChem CID: 12309400; SMILES: $\mathrm{CC} 1(\mathrm{C}=\mathrm{CC} 2=\mathrm{C} 3 \mathrm{C}(=\mathrm{CC}(=\mathrm{C} 2 \mathrm{O} 1) \mathrm{OC}) \mathrm{C}(=\mathrm{O}) \mathrm{C}(=\mathrm{CO} 3)$ $\mathrm{C} 4=\mathrm{CC} 5=\mathrm{C}(\mathrm{C}=\mathrm{C} 4) \mathrm{OCO}$ ) $\mathrm{C}$; InChIKeys: SQBVRNCD BATODN-UHFFFAOYSA-N].

The other large molecule, (X), later identified as (II), contained an ordered portion and a disordered hydrocarbon chain. It was not clear whether the disordered portion of the structure contained a solvent of crystallization, was part of (X), or represented cohabitation of multiple species on the same crystallographic site (Fig. 4). In the absence of additional chemical information it was not possible to model the molecular disorder in $(\mathrm{X})$ because the atomic identities and connectivity were unknown and multiple interpretations of residual electron-density peaks were possible.

The easiest and fastest available analysis that could shed light on the composition of the unknown was mass spectrometry. The positive ion spectrum was dominated by three related ions corresponding to $[(\mathrm{I})+\mathrm{H}]^{+},[2(\mathrm{I})+\mathrm{Na}]^{+}$, and $[3(\mathrm{I})$ $+\mathrm{Na}]^{+}$. The negative ion mass spectrum had one intense ion at 


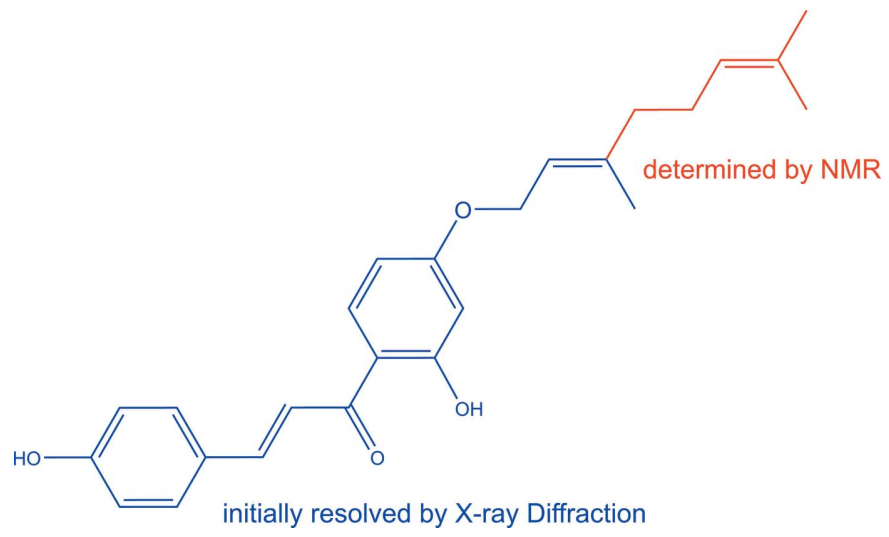

Figure 5

The two portions of (II) identified by single-crystal X-ray analysis and NMR spectroscopy.

$\mathrm{m} / \mathrm{z}$ 391.1918. Its MSMS spectrum showed two major losses to give ions at $\mathrm{m} / z 254.0587$ and 148.0168. The results suggested that molecule $(\mathrm{X})$ was a single disordered species. Unfortunately, the mass of 392 did not yield the possibility of an unambiguous assignment of the disordered atoms in $(\mathrm{X})$; however, this mass information was critical to the success of the subsequent structure identification by NMR. When a structure is to be determined by NMR, two key pieces of information are essential, i.e. the exact mass and the nature of molecular fragments. In this case, both were available; the exact mass of (II) was known and a lion's share of the molecule was identified by X-ray diffractometry.

The crystals of the extract were dissolved in $\mathrm{CDCl}_{3}$ and transferred to a Shigemi tube for NMR analysis. A unique feature of the unknown compound $(\mathrm{X})$ was the allylic phenol ether revealed by partial X-ray structural analysis. This provided a handle that assisted the interpretation of a spectrum composed of the unknown. The $\mathrm{CH}_{2}$ group of the phenol ether was readily identified as a doublet at $4.59 \mathrm{ppm}$ by its chemical shift, multiplicity, and the unique long-range ${ }^{1} \mathrm{H}-{ }^{13} \mathrm{C}$ coupling to the oxygen-bearing $\mathrm{C}$ atom on the aromatic ring at

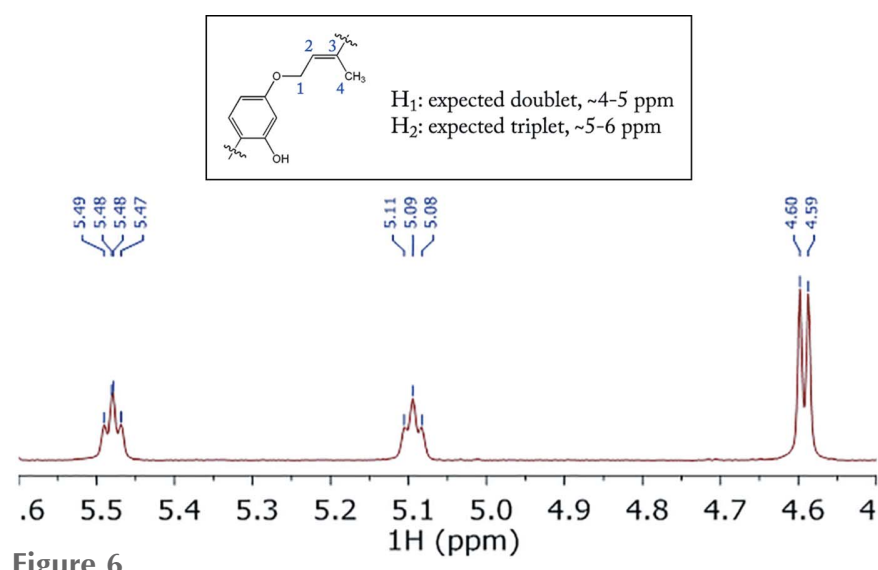

Figure 6

The ${ }^{1} \mathrm{H}$ spectrum of the unassigned alkene and downfield aliphatic region. The chemical shift and multiplicities do not match any ${ }^{1} \mathrm{H}$ atoms expected from species (I) and thus are likely to be part of species (X).

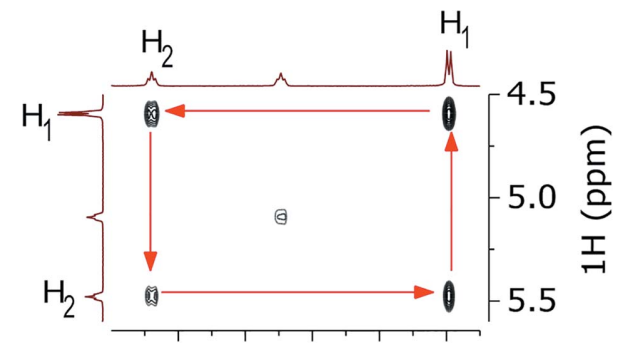

5.45 .25 .04 .84 .6

Figure 7 $1 \mathrm{H}(\mathrm{ppm})$

COSYDQF spectrum of alkene and downfield aliphatic region.

$165.6 \mathrm{ppm}$. From this point, the rest of the molecule was established using ${ }^{1} \mathrm{H}-{ }^{1} \mathrm{H}$ coupling (COSY), ${ }^{1} \mathrm{H}-{ }^{13} \mathrm{C}$ 1-bond and long-range coupling (HSQC and HMBC), and ${ }^{1} \mathrm{H}-{ }^{1} \mathrm{H}$ through-space (NOESY) techniques (Fig. 5). The full structure of the unknown region after the allyl phenol ether functionality is an ethyl chain that terminates in a geminally dimethyl-substituted alkene. Additionally, the spectra match the ${ }^{1} \mathrm{H}$ and ${ }^{13} \mathrm{C}$ spectra reported in the isolation of $4^{\prime}-O$ geranylisoliquiritigenin (Deyou et al., 2015).

In order to determine the unknown portion of species (X), the $\mathrm{CH}_{2}$ and alkene groups of the phenol ether were selected as suitable handles as they were near the unknown portion and possessed a unique chemical shift and coupling pattern that could be easily distinguished from species (I) (Fig. 6).

By combining the partial information from the X-ray diffraction analysis with the exact mass of the compound, we could significantly limit the choices for potential functional groups attached to the allylic fragment. We utilized NMR
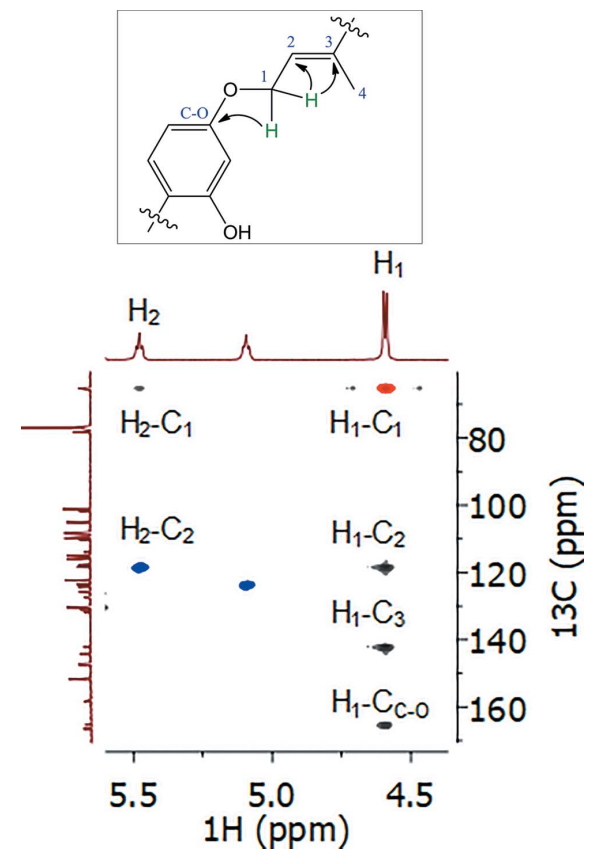

Figure 8

Selected HSQCED and HMBC regions for identifying the boundary region between the $\mathrm{X}$-ray-resolved and unknown regions. HSQCED legend: red is $\mathrm{CH}_{2}$, blue $\mathrm{CH} / \mathrm{CH}_{3}$, and grey $\mathrm{HMBC}$ cross-peaks. 


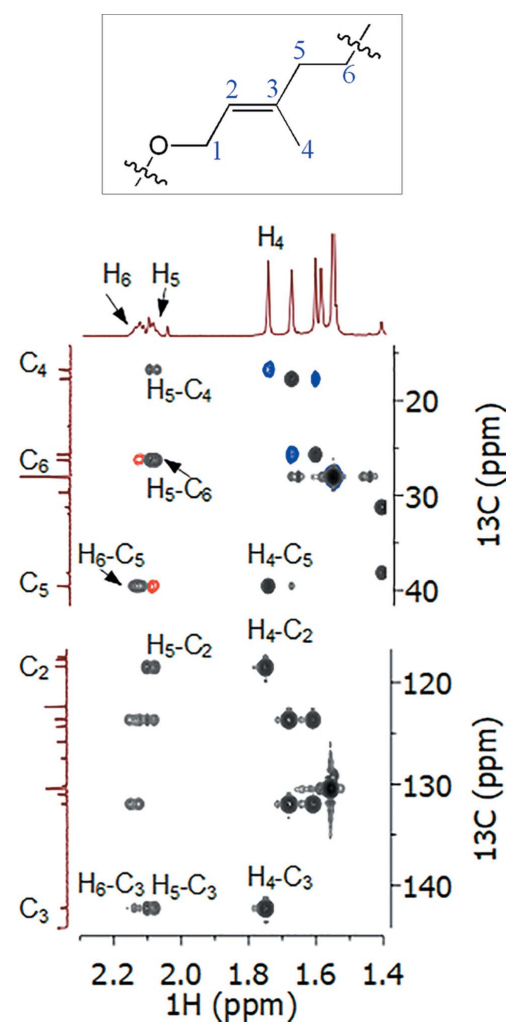

Figure 9

Selected HSQCED and HMBC regions for the determination of the central functional group of the unknown segment. HSQCED legend: red is $\mathrm{CH}_{2}$, blue $\mathrm{CH} / \mathrm{CH}_{3}$, and grey HMBC cross-peaks.

spectroscopy to find fingerprint chemical shifts and connectivity that would either support or eliminate certain options.

Analysis of the ${ }^{1} \mathrm{H}$ spectrum reveals two alkene resonances at 5.48 and $5.09 \mathrm{ppm}$, and a single downfield aliphatic doublet

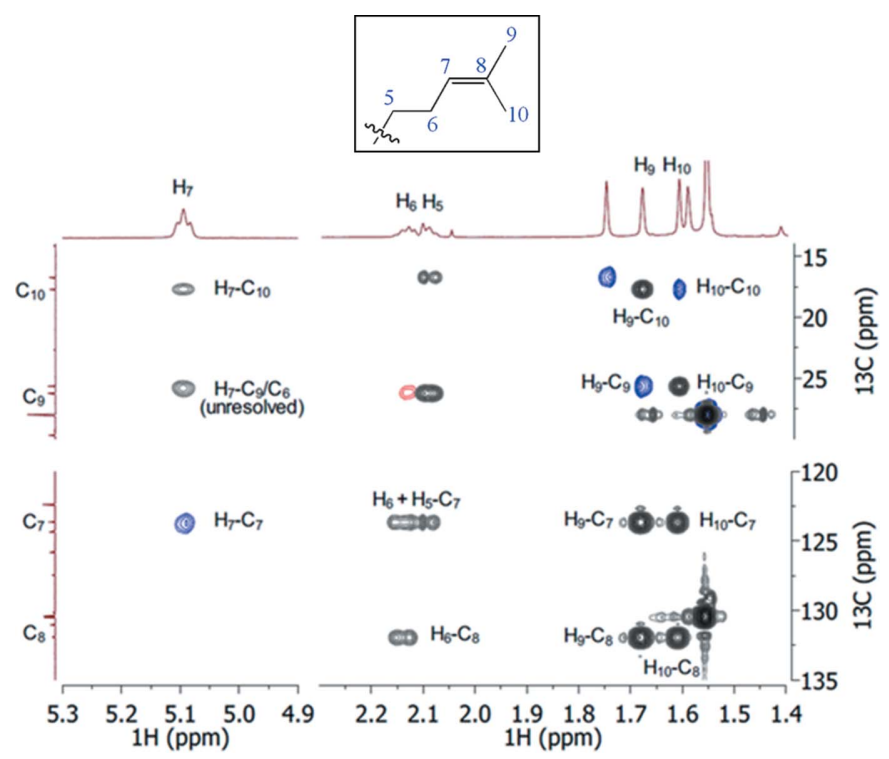

Figure 10

Selected HSQCED and HMBC regions for the determination of the terminal functional groups of the unknown segment. HSQCED legend: red is $\mathrm{CH}_{2}$, blue $\mathrm{CH} / \mathrm{CH}_{3}$, and grey HMBC cross-peaks. at $4.59 \mathrm{ppm}$. The doublet at $4.59 \mathrm{ppm}$ fits the expectation for $\mathrm{H}_{1}$; however, either of the two alkene triplets could fit the description of the $\mathrm{H}_{2}$. Therefore, a COSY experiment was performed to detect ${ }^{1} \mathrm{H}-{ }^{1} \mathrm{H} J$-coupling interactions between the peaks from 4.5 to $5.6 \mathrm{ppm}$. Indeed, cross-peaks were observed between the alkene triplet at $5.48 \mathrm{ppm}$ and the aliphatic doublet at $4.59 \mathrm{ppm}$, further supporting the theory that these are two sets of protons on the phenol ether of species (X) (Fig. 7).

${ }^{1} \mathrm{H}-{ }^{13} \mathrm{C}$-edited HSQC and HMBC experiments were then performed to determine the ${ }^{13} \mathrm{C}$ atoms attached to the ${ }^{1} \mathrm{H}$ atoms, as well as neighboring ${ }^{13} \mathrm{C}$ atoms multiple bonds away to confirm their identity (Fig. 8). In the HSQC spectrum, $\mathrm{H}_{1}$ was observed to have a 1-bond correlation with a ${ }^{13} \mathrm{C}$ at $67.3 \mathrm{ppm}$, consistent with an alcohol or ether. Furthermore, the multiplicity editing indicated that it was a $\mathrm{CH}_{2}$ group. Additionally, $\mathrm{H}_{2}$ correlated to a ${ }^{13} \mathrm{C}$ at $118.5 \mathrm{ppm}$, indicative of an alkene or arene group. These 1-bond correlations provided further evidence of the identification of $\mathrm{H}_{1}$ and $\mathrm{H}_{2}$ as part of the allylic phenol ether, but the strongest evidence were the long-range correlation two quaternary centers of $\mathrm{H}_{1}$, i.e. 142.3 and $165.6 \mathrm{ppm}$. The ${ }^{13} \mathrm{C}$ at $142.3 \mathrm{ppm}$ is $\mathrm{C}_{3}$, confirmed by additional HMBC correlations (vide infra). The ${ }^{13} \mathrm{C}$ signal at $165.6 \mathrm{ppm}$ was a clear indicator that the phenol ether region had been correctly identified because arene $\mathrm{C}$ atoms bearing oxygen substituents uniquely/frequently appear in that region.

To determine the structure of the unknown region, ${ }^{1} \mathrm{H}-{ }^{13} \mathrm{C}$ $\mathrm{HMBC}$ resonances were sought that correlated any additional ${ }^{1} \mathrm{H}$ atoms with $\mathrm{C}_{2}$ or $\mathrm{C}_{3}$. Three resonances $(2.13,2.09$, and $1.75 \mathrm{ppm}$ ) were observed to couple to $\mathrm{C}_{3}$, the quaternary alkene $\mathrm{C}$ atom (Fig. 9). The singlet at $1.75 \mathrm{ppm}$, which inte-
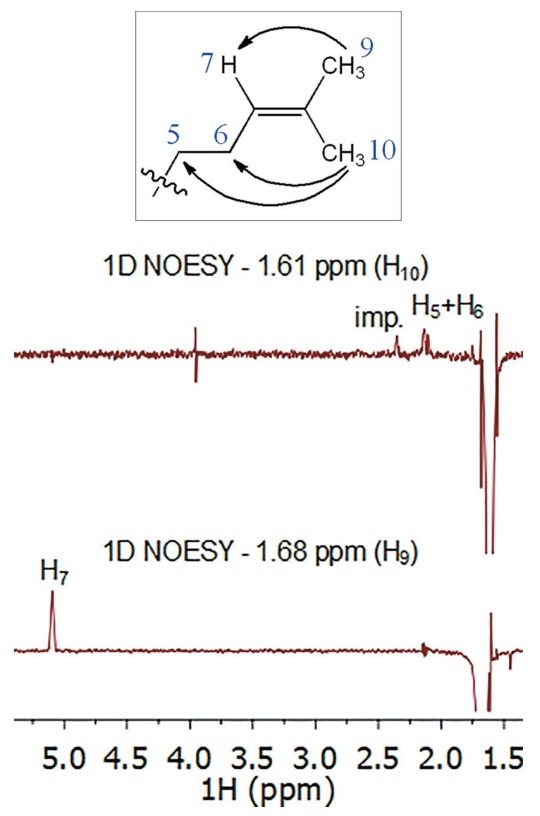

Figure 11 $1 \mathrm{H}(\mathrm{ppm})$

1D NOESY spectra, with the ${ }^{1} \mathrm{H}$ peaks at 1.68 and $1.61 \mathrm{ppm}$ selected. Mix times of $0.5 \mathrm{~s}$ were used in both acquisitions. Artefacts appear as zerointegral (up/down in phase) peaks. A small impurity is found in the top spectrum, likely resulting from the selection of another impurity peak while selecting for $1.61 \mathrm{ppm}$. 


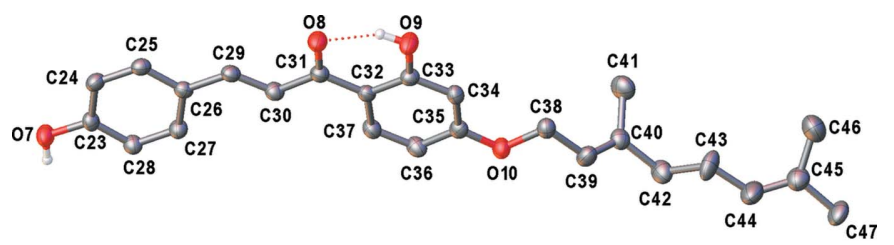

(a)

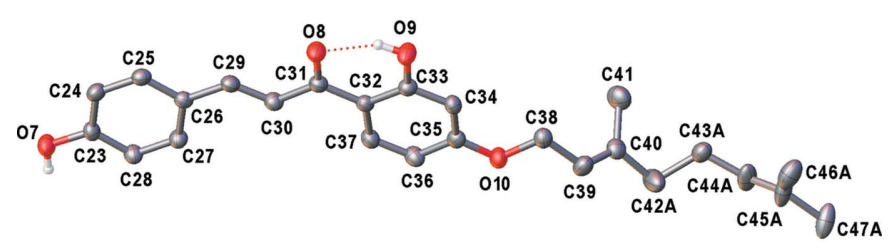

(b)

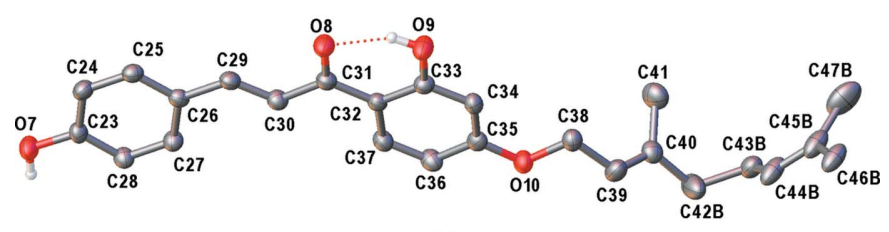

(c)

Figure 12

A molecular drawing of the three conformations of disordered (II), shown with $50 \%$ probability displacement ellipsoids. All $\mathrm{H}$ atoms, except for hydroxy $\mathrm{H}$ atoms, have been omitted. Parts $(a)-(c)$ correspond to the 49.7 (3):26.9 (2):23.4 (3) contributions of the three disorder components.

grates to three protons, couples via a 1-bond to a primary ${ }^{13} \mathrm{C}$ at $16.8 \mathrm{ppm}$ and long range to both $\mathrm{C}_{3}$ and $\mathrm{C}_{2}$, and therefore was assigned as $\mathrm{H}_{4}$. The ${ }^{1} \mathrm{H}$ multiplet at $2.09 \mathrm{ppm}$ also showed long-range couplings with both $\mathrm{C}_{2}$ and $\mathrm{C}_{3}$, indicating that it was next to the alkene group similar to $\mathrm{H}_{4}$. The edited HSQC spectrum revealed that these protons $\left(\mathrm{H}_{5}\right)$ were part of a $\mathrm{CH}_{2}$ group at $39.5 \mathrm{ppm}$ (C5). The assignment of $2.09 \mathrm{ppm}$ to $\mathrm{H}_{5}$ was further supported by $\mathrm{H}_{4}$, showing a 3-bond coupling to $\mathrm{C}_{5}$. Next, the protons at $2.13 \mathrm{ppm}\left(\mathrm{H}_{6}\right)$ were assigned as a $\mathrm{CH}_{2}$ group connected to $\mathrm{H}_{5}$ from the coupling to $\mathrm{C}_{3}$ (3-bonds) and $\mathrm{C}_{5}$ (2-bonds).

The analysis continued from $\mathrm{H}_{6}$ to determine the next functional group on the molecule. Two HMBC correlations to unassigned $\mathrm{C}$ atoms were observed at 123.6 and $132.0 \mathrm{ppm}$. The correlation at $123.6 \mathrm{ppm}$ was also shared with $\mathrm{H}_{5}$, indi- cating that the $\mathrm{C}$ atom $\left(\mathrm{C}_{7}\right)$ is likely immediately adjacent to $\mathrm{H}_{6}$. Analysis of the HSQCED spectrum revealed that the ${ }^{13} \mathrm{C}$ at $123.6 \mathrm{ppm}$ is 1 -bond coupled to the ${ }^{1} \mathrm{H}$ triplet at $5.09 \mathrm{ppm}$ $\left(\mathrm{H}_{7}\right)$. The multiplicity and chemical shift of $\mathrm{H}_{7}$ is indicative of not only an alkene functional group but also that the alkene is trisubstituted due to the lack of additional cis or trans couplings. This is additionally supported by the absence of HSQC correlations to the ${ }^{13} \mathrm{C}$ at $132.0 \mathrm{ppm}$, which was assigned to the quaternary ${ }^{13} \mathrm{C}$, i.e. $\mathrm{C}_{8}$, of the alkene. To determine the two functional groups attached to other end of the alkene $\left(\mathrm{C}_{8}\right)$, HMBC correlations from ${ }^{1} \mathrm{H}$ atoms to $\mathrm{C}_{7}$ and $\mathrm{C}_{8}$ were explored. Only two sets of ${ }^{1} \mathrm{H}$ resonances, 1.68 and $1.61 \mathrm{ppm}$, aside from $\mathrm{H}_{5}$ and $\mathrm{H}_{6}$, had $\mathrm{HMBC}$ correlations to $\mathrm{C}_{7}$ and $\mathrm{C}_{8}$. Each of these ${ }^{1} \mathrm{H}$ resonances were strong singlets and integrated to three protons, which clearly indicated that the alkene was capped with two methyl groups (Fig. 10). The ${ }^{1} \mathrm{H}$ signals at $1.68 \mathrm{ppm}$ were assigned to $\mathrm{H}_{9}$ due to ${ }^{1} \mathrm{H}-{ }^{1} \mathrm{H}$ NOEs between $\mathrm{H}_{7}$ and $\mathrm{H}_{9}$ from a selective 1D NOE experiment (Fig. 11). This assignment was confirmed by looking at NOE correlations from $\mathrm{H}_{10}$, which revealed through-space correlations to $\mathrm{H}_{5}$ and $\mathrm{H}_{6}$ and not $\mathrm{H}_{7}$.

Armed with the exact knowledge of the composition and connectivity of (II) [PubChem CID: 10318361, SMILES: CC$(=\mathrm{CCCC}(=\mathrm{CCOC} 1=\mathrm{CC}(=\mathrm{C}(\mathrm{C}=\mathrm{C} 1) \mathrm{C}(=\mathrm{O}) \mathrm{C}=\mathrm{CC} 2=\mathrm{C}-$ $\mathrm{C}=\mathrm{C}(\mathrm{C}=\mathrm{C} 2) \mathrm{O}) \mathrm{O}) \mathrm{C}) \mathrm{C}$, InChIKeys: YGNHPWQWWZLUBYUHGDPLQBSA-N], we modelled its positional disorder with confidence. The chain at atom $\mathrm{C} 40$ is disordered over three positions in a 49.7 (3):26.9 (2):23.4 (3) ratio (Figs. 12a-c), and was refined with restraints and constraints. The relative location of the remaining small residual peaks of electron density in the difference map were believed to belong to the solvent of crystallization. This molecule of solvent ethanol has a partial occupancy of $13.5(2) \%$ and is disordered over a crystallographic inversion center. This molecule was refined with an idealized geometry (Guzei, 2014) (Fig. 13) (PubChem CID: 702; SMILES: CCO; InChIKeys: LFQSCWFLJHTTHZUHFFFAOYSA-N).

The two symmetry-independent molecules of (I) can be overlaid without inversion, with an s..u. value of $0.260 \AA$ for the non-H atoms. Molecules ( $\mathrm{I} a$ ) and $(\mathrm{I} b)$ both have very similar geometries with minor differences in the positions of

Figure 13

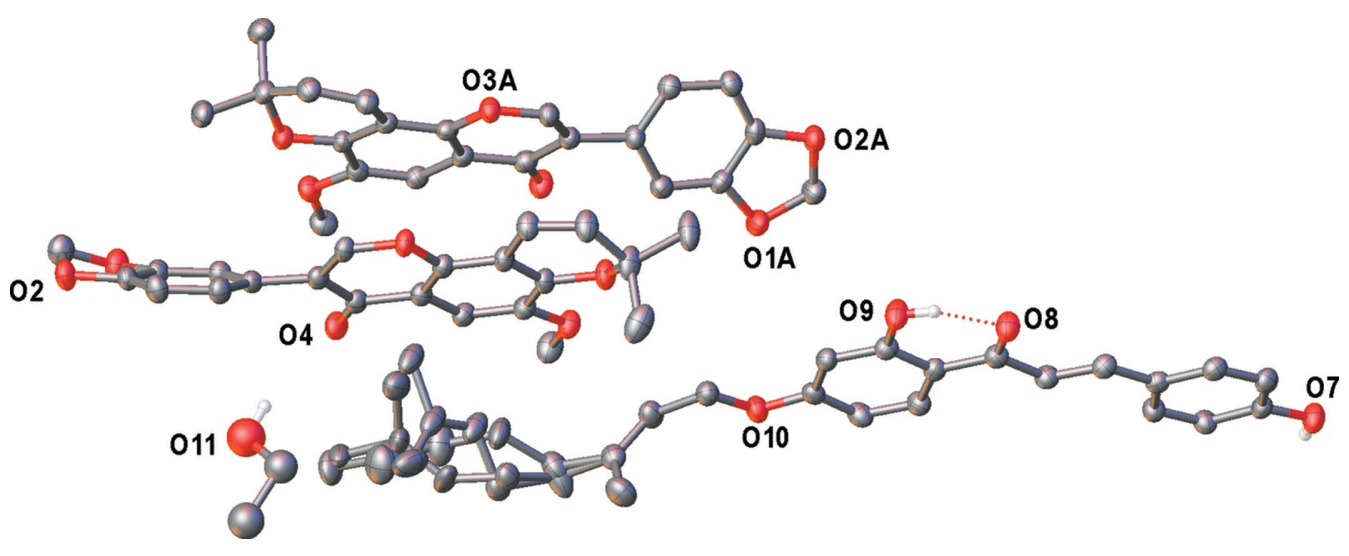

The contents of the asymmetric unit of the 2(I):(II):0.13EtOH cocrystal. All H atoms, except for hydroxy H atoms, have been omitted. Displacement ellipsoids are drawn at the $50 \%$ probability level. 


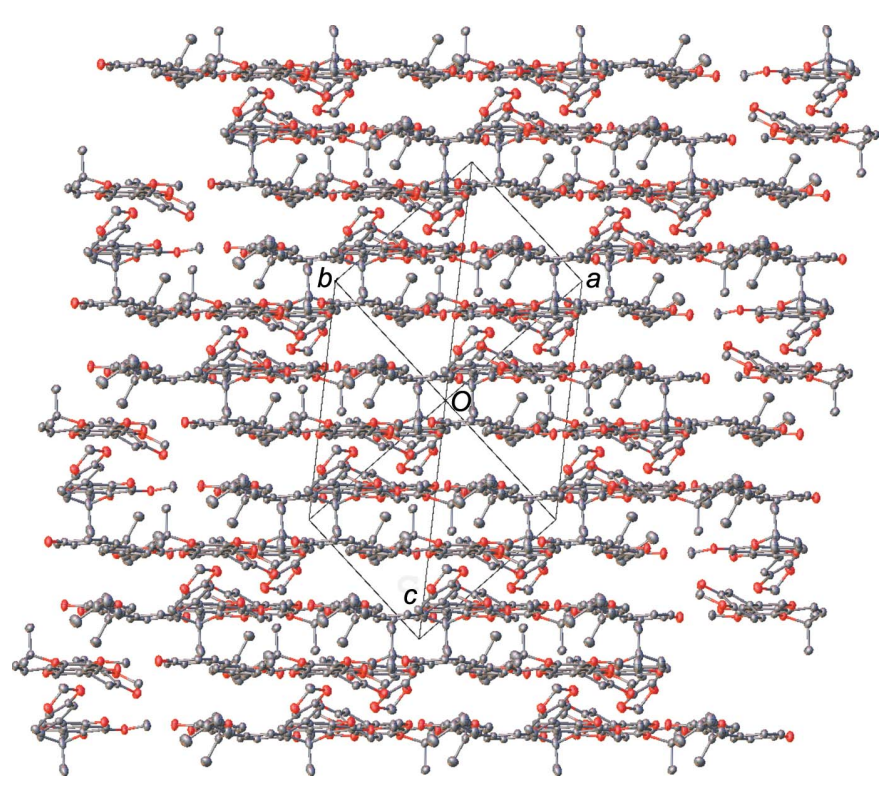

Figure 14

The packing diagram of the 2(I):(II):0.13EtOH cocrystal, viewed along [111]. All $\mathrm{H}$ atoms have been omitted.

the three methyl groups and the main difference being in the location of atoms $\mathrm{C} 1 / \mathrm{C} 1 \mathrm{a}$ that reside on opposite sides of their respective dioxole planes. The dioxole rings are characterized by the Cremer \& Pople parameters (Cremer \& Pople, 1975): $q_{2}=0.244(3) \AA$ and $\varphi_{2}=32.9(6)^{\circ}$ for $(\mathrm{I} a)$, and $q_{2}=$ $0.137(2) \AA$ and $\varphi_{2}=217.3(10)^{\circ}$ in (Ib). The puckering amplitude $q_{2}$ indicates a larger ring distortion in ( $\mathrm{I} a$ ), whereas the phase angle $\varphi_{2}$ is indicative of distorted envelope conformation $E_{2}$ (ideal value $=36^{\circ}$ ) for $(\mathrm{I} a)$ and ${ }^{2} E$ (ideal value $=$ $214^{\circ}$ ) for $(\mathrm{I} b)$. Atoms $\mathrm{C} 1 / \mathrm{Cla}$ are the flap atoms in these envelopes. The dihedral angles between the envelope (O1/C2/ $\mathrm{C} 3 / \mathrm{O} 2)$ and flap $(\mathrm{O} 1 / \mathrm{C} 1 / \mathrm{C} 2)$ planes are $26.4(3)$ and $14.90(17)^{\circ}$ in molecules $(\mathrm{I} a)$ and $(\mathrm{I} b)$. The magnitude of this angle in 304 monosubstituted benzodioxole congeners reported to the Cambridge Structural Database (Groom et al., 2016) ranges between 0 and $29.63^{\circ}$.

The dihedral angles between the arene plane of the benzodioxole and that of the $4 \mathrm{H}$-chromene moiety of the fused three-ring portion in molecules $(\mathrm{I} a)$ and $(\mathrm{I} b)$ are $37.37(8)$ and $39.71(8)^{\circ}$, respectively. The pyran portions of the pyranochromenone ring systems in these molecules cannot be accurately described with one conformational type. Their shapes are closest to screw-boat ${ }^{3} S_{4}$ and envelope ${ }^{3} E$, with atoms $\mathrm{C} 20 / \mathrm{C} 20$ a serving as the flap atoms. The Cremer \& Pople ring puckering coordinates are $Q=0.286$ (3) $\AA, \theta=$ $113.8(6)^{\circ}$, and $\varphi=138.5(6)^{\circ}$ for $(\mathrm{I} a)$, and $Q=0.341$ (2) $\AA$, $\theta=$ $112.2(5)^{\circ}$, and $\varphi=138.0(5)^{\circ}$ for $(\mathrm{I} b)$. The puckering amplitude $Q$ suggests that the ring is more distorted in the latter, but the phase angles $\theta$ and $\varphi$ attest that the overall conformations are essentially identical.

The molecule of (II) consists of an ordered near-planar portion and the disordered chain. In the ordered part, atoms O8-O10 and C29-C38 are coplanar within $0.045 \AA$. This plane forms a dihedral angle of $7.85(10)^{\circ}$ with the phenol ring at atom $\mathrm{C} 29$.
All bond lengths and angles in the reported molecules fall in the usual ranges, as revealed by a MOGUL (Bruno et al., 2004) molecular geometry check.

There are two types of hydrogen-bonding interactions in this structure. The strong interaction $\mathrm{O} 7-\mathrm{H} 7 \cdots \mathrm{O} 4 A(-x+1$, $-y+2,-z+1)$ of type $D(2)$ is observed between molecules (II) and (Ib) at $(-x+1,-y+2,-z+1)$, described with $D \cdots A=2.673(2) \AA$ and $D-\mathrm{H} \cdots A=168.6^{\circ}$. The weak intramolecular $S(6)$ interaction $\mathrm{O} 9-\mathrm{H} 9 \ldots \mathrm{O} 8$ in (II) is characterized with $D \cdots A=2.527$ (2) $\AA$ and $D-\mathrm{H} \cdots A=147.1^{\circ}$.

The molecules of (I) and (II) pack in layers (Fig. 14) with several $\pi-\pi$ interactions between the conjugated systems in all three molecules. The benzopyranone rings in molecules (I $a)$ and $(\mathrm{I} b)$ are nearly coplanar, with a separation of $3.36(4) \AA$. The $\mathrm{C} 30-\mathrm{C} 37 / \mathrm{O} 8 / \mathrm{O} 9$ fragment of (II) forms a stacking interaction with its symmetry-related mate (II) at $(-x+1,-y+2$, $-z+1)$, to which it is parallel, with an interplanar distance of 3.349 (2) $\AA$ and with the benzopyranone portion of $(\mathrm{I} b)$ at $(x$, $y+1, z)$ at $3.3504(17) \AA$.

\section{Conclusion}

The structural characterization of a natural product, a twocomponent extract of unknown composition, was conducted by standard crystallographic techniques, but would not have been possible without data obtained by $2 \mathrm{D}$ NMR spectroscopy and mass spectrometry. The cocrystal in question contained three components, namely two ordered molecules of (I), a disordered molecule of (II), and a partially occupied molecule of crystallization solvent, i.e. ethanol. The positional disorder in (II) required the use of a 2D NMR technique to establish the atomic identities and connectivity in the disordered hydrocarbon chain. The NMR results were confirmed by MS and literature data. A subsequent atomic disorder modelling allowed the crystallographic refinement of (II) to be completed. The remaining peaks of residual electron density were interpreted as a partially occupied solvent of crystallization.

We are currently developing a separation protocol for this compound mixture with the goal of testing the individual components for analgesic, antimalarial, and anti-inflammatory properties.

\section{Acknowledgements}

NIH provided the funds to purchase the Thermo Q Exactive $^{\mathrm{TM}}$ Plus. Crystallographic and NMR instrumentation at UW-Madison is supported by a generous gift from the Paul J. Bender fund. The authors thank Dr Charles G. Fry (UWMadison) for help with the NMR studies of (I) and (II), and Dr Galina A. Bikzhanova (LabCorp) for fruitful discussions.

\section{Funding information}

Funding for this research was provided by: National Institutes of Health (award No. 1S10 OD020022-1); Paul J. Bender fund. 


\section{References}

Adelekan, A. M., Prozesky, E. A., Hussein, A. A., Ureña, L. D., van Rooyen, P. H., Liles, D. C., Meyer, J. J. M. \& Rodríguez, B. (2008). J. Nat. Prod. 71, 1919-1922.

Berger, S. \& Braun, S. (2004). In 200 and More NMR Experiments. Weinheim: Wiley-VCH.

Bruker (2012). SADABS. Bruker AXS Inc., Madison, Wisconsin, USA.

Bruker (2016). APEX3 and SAINT-Plus. Bruker AXS Inc., Madison, Wisconsin, USA.

Bruno, I. J., Cole, J. C., Kessler, M., Luo, J., Motherwell, W. D. S., Purkis, L. H., Smith, B. R., Taylor, R., Cooper, R. I., Harris, S. E. \& Orpen, A. G. (2004). J. Chem. Inf. Comput. Sci. 44, 2133-2144.

Claridge, T. D. W. (2016). In High-Resolution NMR Techniques in Organic Chemistry, 3rd ed. Amsterdam: Elsevier.

Cremer, D. \& Pople, J. A. (1975). J. Am. Chem. Soc. 97, 1354-1358.

Deyou, T., Gumula, I., Pang, F., Gruhonjic, A., Mumo, M., Holleran, J., Duffy, S., Fitzpatrick, P. A., Heydenreich, M., Landberg, G., Derese, S., Avery, V., Rissanen, K., Erdélyi, M. \& Yenesew, A. (2015). J. Nat. Prod. 78, 2932-2939.

Dolomanov, O. V., Bourhis, L. J., Gildea, R. J., Howard, J. A. K. \& Puschmann, H. (2009). J. Appl. Cryst. 42, 339-341.

Fotsing, M. T., Yankep, E., Njamen, D., Fomum, Z. T., Nyasse, B., Bodo, B., Recio, M. C., Giner, R. M. \& Ríos, J.-L. (2003). Planta Med. 69, 767-770.

Friebolin, H. (2010). In Basic One- and Two-Dimensional NMR Spectroscopy, 5th ed. Weinheim: VCH.

Groom, C. R., Bruno, I. J., Lightfoot, M. P. \& Ward, S. C. (2016). Acta Cryst. B72, 171-179.
Guzei, I. A. (2014). J. Appl. Cryst. 47, 806-809.

Krause, L., Herbst-Irmer, R., Sheldrick, G. M. \& Stalke, D. (2015). J. Appl. Cryst. 48, 3-10.

Lemos, V. S., Côrtes, S. F., dos Santos, M. H., Ellena, J., Moreira, M. E. C. \& Doriguetto, A. C. (2006). Chem. Biodivers. 3, 635645.

Liang, G., Xu, B., Pan, W., Cao, P., Zhang, Y., Lu, Y., Wu, Y. \& Hao, X. (2009). Nat. Prod. Res. 23, 1-4.

Ngounou, F. N., Meli, A. L., Lontsi, D., Sondengam, B. L., Atta-UrRahman Choudhary, M. I., Malik, S. \& Akhtar, F. (2000). Phytochemistry, 54, 107-110.

Parveen, M., Ahmad, F., Malla, A. M., Khan, M. S., Rehman, S. U., Tabish, M., Silva, M. R. \& Silva, P. S. P. (2016). J. Photochem. Photobiol. B, 159, 218-228.

Parveen, M., Azaz, S., Zafar, A., Ahmad, F., Silva, M. R. \& Silva, P. S. P. (2017). J. Photochem. Photobiol. B, 167, 176-188.

Riaz, M., Krohn, K., Malik, A. \& Flörke, U. (2004). Chem. Biodivers. 1, 458-462.

Saleem, M., Nazir, M., Ali, M. S., Hussain, H., Lee, Y. S., Riaz, N. \& Jabbar, A. (2010). Nat. Prod. Rep. 27, 238-254.

Sheldrick, G. M. (2015a). Acta Cryst. A71, 3-8.

Sheldrick, G. M. (2015b). Acta Cryst. C71, 3-8.

Simard, F., Legault, J., Lavoie, S. \& Pichette, A. (2014). Phytochemistry, 100, 141-149.

Socolsky, C., Asakawa, Y. \& Bardón, A. (2007). J. Nat. Prod. 70, 1837-1845.

Veitch, N. C. (2007). Nat. Prod. Rep. 24, 417-464.

Veitch, N. C. (2009). Nat. Prod. Rep. 26, 776-802.

Veitch, N. C. (2013). Nat. Prod. Rep. 30, 988-1027. 


\section{supporting information}

Acta Cryst. (2017). C73, 994-1002 [https://doi.org/10.1107/S2053229617014966]

Deciphering composition and connectivity of a natural product with the assistance of MS and 2D NMR

Anastasiya I. Vinokur, Paul B. White, Maurice Tagatsing Fotsing, Charmaine Arderne, Derek

Tantoh Ndinteh, Martha M. Vestling and Ilia A. Guzei

Computing details

Data collection: APEX3 (Bruker, 2016); cell refinement: SAINT-Plus (Bruker, 2016); data reduction: SAINT-Plus (Bruker, 2016); program(s) used to solve structure: SHELXT (Sheldrick, 2015a); program(s) used to refine structure:

SHELXL2016 (Sheldrick, 2015b); molecular graphics: OLEX2 (Dolomanov et al., 2009); software used to prepare material for publication: OLEX2 (Dolomanov et al., 2009).

3-(2H-1,3-Benzodioxol-5-yl)-6-methoxy-8,8-dimethyl-4H,8H-pyrano[2,3-h]chromen-4-one- (2E)-1-(4-\{[(2E)-3,7dimethylocta-2,6-dien-1-yl]oxy\}-2-hydroxyphenyl)-3-(4-hydroxyphenyl)prop-2-en-1-one-ethanol (2/1/0.13)

Crystal data

$2 \mathrm{C}_{22} \mathrm{H}_{18} \mathrm{O}_{6} \cdot \mathrm{C}_{25} \mathrm{H}_{28} \mathrm{O}_{4} \cdot 0.13 \mathrm{C}_{2} \mathrm{H}_{6} \mathrm{O}$

$M_{r}=1155.42$

Triclinic, $P \overline{1}$

$a=12.519(3) \AA$

$b=12.594(3) \AA$

$c=19.975(4) \AA$

$\alpha=80.12(3)^{\circ}$

$\beta=89.69(3)^{\circ}$

$\gamma=68.02(3)^{\circ}$

$V=2871.3(12) \AA^{3}$

Data collection

Bruker APEX II Quazar

diffractometer

Radiation source: microfocus sealed X-ray tube, Incoatec I $\mu \mathrm{s}$

Mirror optics monochromator

Detector resolution: 7.9 pixels $\mathrm{mm}^{-1}$

$0.5^{\circ} \omega$ and $0.5^{\circ} \varphi$ scans

Absorption correction: multi-scan

(SADABS; Krause et al., 2015)

Refinement

Refinement on $F^{2}$

Least-squares matrix: full

$R\left[F^{2}>2 \sigma\left(F^{2}\right)\right]=0.057$

$w R\left(F^{2}\right)=0.165$

$S=1.03$
$Z=2$

$F(000)=1219$

$D_{\mathrm{x}}=1.336 \mathrm{Mg} \mathrm{m}^{-3}$

Mo $K \alpha$ radiation, $\lambda=0.71073 \AA$

Cell parameters from 6163 reflections

$\theta=2.5-25.2^{\circ}$

$\mu=0.10 \mathrm{~mm}^{-1}$

$T=100 \mathrm{~K}$

Block, yellow

$0.11 \times 0.06 \times 0.02 \mathrm{~mm}$

$T_{\min }=0.679, T_{\max }=0.745$

42663 measured reflections

11806 independent reflections

7371 reflections with $I>2 \sigma(I)$

$R_{\text {int }}=0.054$

$\theta_{\text {max }}=26.5^{\circ}, \theta_{\text {min }}=1.0^{\circ}$

$h=-15 \rightarrow 15$

$k=-15 \rightarrow 15$

$l=-25 \rightarrow 24$

11806 reflections

871 parameters

193 restraints

Primary atom site location: structure-invariant direct methods 
Hydrogen site location: inferred from neighbouring sites

H-atom parameters constrained

$$
\begin{aligned}
& w=1 /\left[\sigma^{2}\left(F_{\mathrm{o}}^{2}\right)+(0.0815 P)^{2}+0.7244 P\right] \\
& \text { where } P=\left(F_{\mathrm{o}}^{2}+2 F_{\mathrm{c}}^{2}\right) / 3 \\
& (\Delta / \sigma)_{\max }=0.001 \\
& \Delta \rho_{\max }=0.47 \mathrm{e} \AA^{-3} \\
& \Delta \rho_{\min }=-0.55 \mathrm{e} \AA^{-3}
\end{aligned}
$$

Special details

Geometry. All esds (except the esd in the dihedral angle between two 1.s. planes) are estimated using the full covariance

\begin{tabular}{|c|c|c|c|c|c|}
\hline & $x$ & $y$ & $z$ & $U_{\text {iso }} * / U_{\text {eq }}$ & Occ. $(<1)$ \\
\hline $\mathrm{O} 1$ & $0.56890(15)$ & $-0.13223(16)$ & $0.00339(10)$ & $0.0432(5)$ & \\
\hline $\mathrm{O} 2$ & $0.70046(14)$ & $-0.24504(15)$ & $-0.06278(9)$ & $0.0368(4)$ & \\
\hline $\mathrm{O} 3$ & $1.00320(13)$ & $0.02748(13)$ & $0.14992(9)$ & $0.0293(4)$ & \\
\hline $\mathrm{O} 4$ & $0.66409(14)$ & $0.18670(14)$ & $0.07879(9)$ & $0.0353(4)$ & \\
\hline $\mathrm{O} 5$ & $0.75708(13)$ & $0.44891(14)$ & $0.22375(9)$ & $0.0337(4)$ & \\
\hline O6 & $0.97623(13)$ & $0.34022(14)$ & $0.26052(9)$ & $0.0341(4)$ & \\
\hline $\mathrm{C} 1$ & $0.6006(2)$ & $-0.2377(2)$ & $-0.02497(15)$ & $0.0434(7)$ & \\
\hline H1A & 0.618844 & -0.306631 & 0.011829 & $0.052 *$ & \\
\hline H1B & 0.536444 & -0.233931 & -0.055303 & $0.052 *$ & \\
\hline $\mathrm{C} 2$ & 0.66991 (19) & $-0.1149(2)$ & $0.00882(12)$ & $0.0285(5)$ & \\
\hline $\mathrm{C} 3$ & $0.7481(2)$ & $-0.1809(2)$ & $-0.03163(12)$ & $0.0307(6)$ & \\
\hline $\mathrm{C} 4$ & $0.8545(2)$ & $-0.1757(2)$ & $-0.03981(13)$ & $0.0354(6)$ & \\
\hline $\mathrm{H} 4$ & 0.906794 & -0.218605 & -0.069078 & $0.043^{*}$ & \\
\hline $\mathrm{C} 5$ & $0.8824(2)$ & $-0.1037(2)$ & -0.00280 & $0.0332(6)$ & \\
\hline H5 & 0.955893 & -0.098336 & -0.006982 & $0.040^{*}$ & \\
\hline C6 & $0.8059(2)$ & $-0.0394(2)$ & $0.04013(12)$ & $0.0281(5)$ & \\
\hline $\mathrm{C} 7$ & $0.6956(2)$ & $-0.0442(2)$ & $0.04540(12)$ & $0.0286(5)$ & \\
\hline H7A & 0.641183 & -0.000145 & 0.073305 & $0.034 *$ & \\
\hline $\mathrm{C} 8$ & $0.8440(2)$ & $0.0263(2)$ & $0.08318(12)$ & $0.0276(5)$ & \\
\hline C9 & $0.9531(2)$ & $-0.0215(2)$ & $0.11070(12)$ & $0.0286(5)$ & \\
\hline H9A & 0.999426 & -0.096313 & 0.101903 & $0.034 *$ & \\
\hline $\mathrm{C} 10$ & $0.9372(2)$ & $0.1349(2)$ & $0.16427(12)$ & $0.0275(5)$ & \\
\hline C11 & $0.82200(19)$ & $0.1910(2)$ & $0.14116(12)$ & $0.0270(5)$ & \\
\hline $\mathrm{C} 12$ & $0.7671(2)$ & $0.1389(2)$ & $0.09904(13)$ & $0.0286(5)$ & \\
\hline $\mathrm{C} 13$ & $0.7590(2)$ & $0.2988(2)$ & $0.16075(13)$ & $0.0287(5)$ & \\
\hline H13 & 0.679674 & 0.338508 & 0.146223 & $0.034^{*}$ & \\
\hline $\mathrm{C} 14$ & $0.8110(2)$ & $0.3469(2)$ & $0.20055(13)$ & $0.0291(5)$ & \\
\hline $\mathrm{C} 15$ & $0.6354(2)$ & $0.5050(2)$ & $0.20840(16)$ & $0.0410(7)$ & \\
\hline $\mathrm{H} 15 \mathrm{~A}$ & 0.619472 & 0.527343 & 0.158920 & $0.061^{*}$ & \\
\hline H15B & 0.605461 & 0.574801 & 0.229127 & $0.061 *$ & \\
\hline $\mathrm{H} 15 \mathrm{C}$ & 0.597787 & 0.451518 & 0.226612 & $0.061 *$ & \\
\hline C16 & $0.9300(2)$ & $0.2893(2)$ & $0.22067(12)$ & $0.0284(5)$ & \\
\hline $\mathrm{C} 17$ & $0.99329(19)$ & $0.1816(2)$ & $0.20388(12)$ & $0.0273(5)$ & \\
\hline $\mathrm{C} 18$ & $1.1118(2)$ & $0.1207(2)$ & $0.23238(13)$ & $0.0334(6)$ & \\
\hline
\end{tabular}
matrix. The cell esds are taken into account individually in the estimation of esds in distances, angles and torsion angles; correlations between esds in cell parameters are only used when they are defined by crystal symmetry. An approximate (isotropic) treatment of cell esds is used for estimating esds involving l.s. planes.

Fractional atomic coordinates and isotropic or equivalent isotropic displacement parameters $\left(\AA^{2}\right)$ 


\begin{tabular}{|c|c|c|c|c|}
\hline H18 & 1.153249 & 0.042039 & 0.228084 & $0.040 *$ \\
\hline $\mathrm{C} 19$ & $1.1612(2)$ & $0.1760(2)$ & $0.26440(14)$ & $0.0386(7)$ \\
\hline H19 & 1.236048 & 0.133875 & 0.286257 & $0.046^{*}$ \\
\hline $\mathrm{C} 20$ & $1.1028(2)$ & $0.3033(2)$ & $0.26724(15)$ & $0.0398(7)$ \\
\hline C21 & $1.1258(2)$ & $0.3311(3)$ & $0.33547(17)$ & $0.0508(8)$ \\
\hline $\mathrm{H} 21 \mathrm{~A}$ & 1.079024 & 0.412859 & 0.336650 & $0.076^{*}$ \\
\hline $\mathrm{H} 21 \mathrm{~B}$ & 1.207773 & 0.317551 & 0.341687 & $0.076^{*}$ \\
\hline $\mathrm{H} 21 \mathrm{C}$ & 1.105471 & 0.280869 & 0.372098 & $0.076^{*}$ \\
\hline C22 & $1.1381(2)$ & $0.3742(2)$ & 0.20857 (18) & $0.0540(9)$ \\
\hline $\mathrm{H} 22 \mathrm{~A}$ & 1.113083 & 0.361148 & 0.165364 & $0.081 *$ \\
\hline $\mathrm{H} 22 \mathrm{~B}$ & 1.222209 & 0.350322 & 0.211383 & $0.081^{*}$ \\
\hline $\mathrm{H} 22 \mathrm{C}$ & 1.101768 & 0.457096 & 0.211032 & $0.081^{*}$ \\
\hline O1A & $0.79383(15)$ & $0.55491(14)$ & $0.44566(9)$ & $0.0376(4)$ \\
\hline $\mathrm{O} 2 \mathrm{~A}$ & $0.90429(14)$ & $0.47814(14)$ & $0.54799(9)$ & $0.0338(4)$ \\
\hline $\mathrm{O} 3 \mathrm{~A}$ & $0.98022(13)$ & $-0.01385(13)$ & $0.34200(8)$ & $0.0299(4)$ \\
\hline $\mathrm{O} 4 \mathrm{~A}$ & $0.68874(14)$ & $0.27032(14)$ & $0.35312(9)$ & $0.0353(4)$ \\
\hline $\mathrm{O} 5 \mathrm{~A}$ & $0.58184(13)$ & $0.02020(14)$ & $0.20800(9)$ & $0.0324(4)$ \\
\hline O6A & $0.77140(13)$ & $-0.16003(13)$ & $0.20853(8)$ & $0.0298(4)$ \\
\hline $\mathrm{C} 1 \mathrm{~A}$ & $0.8164(2)$ & $0.5802(2)$ & $0.51056(13)$ & $0.0364(6)$ \\
\hline H1AA & 0.842550 & 0.646219 & 0.503883 & $0.044^{*}$ \\
\hline H1AB & 0.745350 & 0.601687 & 0.535757 & $0.044^{*}$ \\
\hline $\mathrm{C} 2 \mathrm{~A}$ & $0.8450(2)$ & $0.4352(2)$ & $0.45289(13)$ & $0.0296(6)$ \\
\hline $\mathrm{C} 3 \mathrm{~A}$ & $0.9101(2)$ & $0.3899(2)$ & $0.51380(13)$ & $0.0291(5)$ \\
\hline $\mathrm{C} 4 \mathrm{~A}$ & 0.9707 (2) & $0.2733(2)$ & $0.53390(13)$ & $0.0308(6)$ \\
\hline $\mathrm{H} 4 \mathrm{~A}$ & 1.016927 & 0.242621 & 0.575523 & $0.037^{*}$ \\
\hline $\mathrm{C} 5 \mathrm{~A}$ & $0.9614(2)$ & $0.2010(2)$ & $0.49004(12)$ & $0.0296(6)$ \\
\hline H5A & 1.000597 & 0.119114 & 0.503025 & $0.035^{*}$ \\
\hline C6A & $0.89666(19)$ & $0.2453(2)$ & $0.42816(12)$ & $0.0277(5)$ \\
\hline C7A & $0.8371(2)$ & $0.3669(2)$ & $0.40805(13)$ & $0.0310(6)$ \\
\hline H7AA & 0.793779 & 0.399680 & 0.365550 & $0.037^{*}$ \\
\hline C8A & $0.8879(2)$ & $0.16453(19)$ & $0.38420(12)$ & $0.0278(5)$ \\
\hline C9A & $0.9806(2)$ & $0.0690(2)$ & $0.37731(12)$ & $0.0292(5)$ \\
\hline H9AA & 1.052004 & 0.058926 & 0.398962 & $0.035^{*}$ \\
\hline $\mathrm{C} 10 \mathrm{~A}$ & $0.87817(19)$ & -0.00108 (19) & $0.30995(12)$ & $0.0262(5)$ \\
\hline $\mathrm{C} 11 \mathrm{~A}$ & $0.77769(19)$ & $0.09522(19)$ & $0.31192(12)$ & $0.0260(5)$ \\
\hline $\mathrm{C} 12 \mathrm{~A}$ & $0.7781(2)$ & $0.1837(2)$ & $0.34963(13)$ & $0.0290(5)$ \\
\hline $\mathrm{C} 13 \mathrm{~A}$ & $0.6763(2)$ & $0.1053(2)$ & $0.27681(12)$ & $0.0279(5)$ \\
\hline H13A & 0.607789 & 0.172158 & 0.276554 & $0.033 *$ \\
\hline C14A & $0.67513(19)$ & $0.0199(2)$ & $0.24300(12)$ & $0.0270(5)$ \\
\hline $\mathrm{C} 15 \mathrm{~A}$ & $0.4798(2)$ & $0.1244(2)$ & $0.20150(14)$ & $0.0372(6)$ \\
\hline H15D & 0.418970 & 0.116261 & 0.174665 & $0.056^{*}$ \\
\hline H15E & 0.497158 & 0.190853 & 0.178559 & $0.056^{*}$ \\
\hline $\mathrm{H} 15 \mathrm{~F}$ & 0.453737 & 0.137283 & 0.246825 & $0.056^{*}$ \\
\hline $\mathrm{C} 16 \mathrm{~A}$ & $0.7777(2)$ & $-0.07962(19)$ & $0.24395(12)$ & $0.0266(5)$ \\
\hline $\mathrm{C} 17 \mathrm{~A}$ & $0.8803(2)$ & -0.08961 (19) & $0.27518(12)$ & $0.0267(5)$ \\
\hline C18A & 0.9849 (2) & $-0.1868(2)$ & 0.26507 (13) & $0.0314(6)$ \\
\hline H18A & 1.058771 & -0.188249 & 0.277146 & $0.038^{*}$ \\
\hline C19A & $0.9753(2)$ & $-0.2732(2)$ & $0.23875(13)$ & $0.0333(6)$ \\
\hline
\end{tabular}




\begin{tabular}{|c|c|c|c|c|}
\hline H19A & 1.043151 & -0.332796 & 0.228354 & $0.040 *$ \\
\hline $\mathrm{C} 20 \mathrm{~A}$ & 0.8599 (2) & $-0.2789(2)$ & 0.22507 (13) & $0.0315(6)$ \\
\hline $\mathrm{C} 21 \mathrm{~A}$ & 0.8545 (2) & $-0.3305(2)$ & $0.16251(13)$ & $0.0364(6)$ \\
\hline $\mathrm{H} 21 \mathrm{D}$ & 0.775249 & -0.323836 & 0.153119 & $0.055^{*}$ \\
\hline $\mathrm{H} 21 \mathrm{E}$ & 0.906153 & -0.412820 & 0.170647 & $0.055^{*}$ \\
\hline $\mathrm{H} 21 \mathrm{~F}$ & 0.878576 & -0.288298 & 0.123353 & $0.055^{*}$ \\
\hline $\mathrm{C} 22 \mathrm{~A}$ & $0.8254(2)$ & $-0.3424(2)$ & $0.28855(14)$ & $0.0382(6)$ \\
\hline $\mathrm{H} 22 \mathrm{D}$ & 0.825818 & -0.303553 & 0.327017 & $0.057^{*}$ \\
\hline $\mathrm{H} 22 \mathrm{E}$ & 0.880521 & -0.423302 & 0.299246 & $0.057^{*}$ \\
\hline $\mathrm{H} 22 \mathrm{~F}$ & 0.747856 & -0.341044 & 0.280085 & $0.057^{*}$ \\
\hline $\mathrm{O} 7$ & $0.53894(15)$ & $1.63181(14)$ & $0.67718(10)$ & $0.0380(4)$ \\
\hline H7 & 0.468312 & 1.671304 & 0.667639 & $0.057^{*}$ \\
\hline O8 & $0.78105(14)$ & $1.01492(14)$ & $0.51723(9)$ & $0.0370(4)$ \\
\hline O9 & 0.76654 (14) & $0.86116(14)$ & $0.45556(10)$ & $0.0388(5)$ \\
\hline H9 & 0.797743 & 0.892645 & 0.478773 & $0.058^{*}$ \\
\hline $\mathrm{C} 23$ & 0.5718 (2) & $1.53663(19)$ & $0.64682(12)$ & $0.0282(5)$ \\
\hline C24 & $0.6866(2)$ & $1.4602(2)$ & $0.65693(13)$ & $0.0314(6)$ \\
\hline $\mathrm{H} 24$ & 0.739534 & 1.475183 & 0.684138 & $0.038^{*}$ \\
\hline $\mathrm{C} 25$ & $0.7233(2)$ & $1.3625(2)$ & $0.62722(12)$ & $0.0293(6)$ \\
\hline $\mathrm{H} 25$ & 0.802016 & 1.311165 & 0.634110 & $0.035^{*}$ \\
\hline $\mathrm{C} 26$ & 0.64824 (19) & 1.33647 (19) & $0.58729(12)$ & $0.0272(5)$ \\
\hline $\mathrm{C} 27$ & $0.5329(2)$ & $1.4154(2)$ & $0.57716(13)$ & $0.0301(6)$ \\
\hline $\mathrm{H} 27$ & 0.479899 & 1.400792 & 0.549785 & $0.036^{*}$ \\
\hline $\mathrm{C} 28$ & 0.4954 (2) & $1.5138(2)$ & $0.60640(12)$ & $0.0304(6)$ \\
\hline $\mathrm{H} 28$ & 0.417069 & 1.566226 & 0.598909 & $0.036^{*}$ \\
\hline $\mathrm{C} 29$ & 0.6899 (2) & $1.2295(2)$ & $0.55953(12)$ & $0.0295(6)$ \\
\hline H29 & 0.768398 & 1.179834 & 0.570814 & $0.035^{*}$ \\
\hline $\mathrm{C} 30$ & 0.6292 (2) & $1.1936(2)$ & $0.51978(12)$ & $0.0298(6)$ \\
\hline H30 & 0.551487 & 1.242765 & 0.505577 & $0.036^{*}$ \\
\hline C31 & $0.6789(2)$ & $1.0812(2)$ & 0.49754 (13) & $0.0291(5)$ \\
\hline C32 & $0.61076(19)$ & $1.0458(2)$ & $0.45356(12)$ & $0.0269(5)$ \\
\hline $\mathrm{C} 33$ & $0.65832(19)$ & 0.9360 (2) & 0.43389 (13) & $0.0288(5)$ \\
\hline $\mathrm{C} 34$ & $0.5966(2)$ & $0.8986(2)$ & $0.39197(12)$ & $0.0284(5)$ \\
\hline H34 & 0.630598 & 0.824025 & 0.379703 & $0.034^{*}$ \\
\hline C35 & $0.4854(2)$ & $0.9712(2)$ & $0.36846(12)$ & $0.0291(5)$ \\
\hline C36 & 0.4349 (2) & $1.0800(2)$ & $0.38788(13)$ & $0.0340(6)$ \\
\hline H36 & 0.357582 & 1.128881 & 0.372585 & $0.041 *$ \\
\hline $\mathrm{O} 10$ & $0.41744(14)$ & $0.94625(14)$ & $0.32554(9)$ & $0.0337(4)$ \\
\hline C37 & $0.4967(2)$ & $1.1160(2)$ & $0.42881(13)$ & $0.0314(6)$ \\
\hline H37 & 0.461489 & 1.190576 & 0.440911 & $0.038^{*}$ \\
\hline C38 & $0.4684(2)$ & $0.8399(2)$ & $0.29848(13)$ & $0.0325(6)$ \\
\hline $\mathrm{H} 38 \mathrm{~A}$ & 0.497203 & 0.771654 & 0.335994 & $0.039 *$ \\
\hline H38B & 0.533826 & 0.842004 & 0.270963 & $0.039 *$ \\
\hline C39 & $0.3756(2)$ & $0.8323(2)$ & $0.25516(13)$ & $0.0380(6)$ \\
\hline H39 & 0.311423 & 0.823963 & 0.277764 & $0.046^{*}$ \\
\hline $\mathrm{C} 40$ & 0.3734 (2) & 0.8359 (2) & 0.18817 (13) & $0.0340(6)$ \\
\hline C41 & $0.4659(3)$ & $0.8484(3)$ & $0.14387(15)$ & $0.0494(8)$ \\
\hline $\mathrm{H} 41 \mathrm{~A}$ & 0.524434 & 0.859567 & 0.171055 & $0.074 *$ \\
\hline
\end{tabular}




\begin{tabular}{|c|c|c|c|c|c|}
\hline H41B & 0.432172 & 0.915845 & 0.107096 & $0.074 *$ & \\
\hline $\mathrm{H} 41 \mathrm{C}$ & 0.501715 & 0.777888 & 0.124338 & $0.074^{*}$ & \\
\hline $\mathrm{C} 42$ & 0.2788 (4) & $0.8119(7)$ & $0.1549(6)$ & $0.043(3)$ & $0.497(3)$ \\
\hline $\mathrm{H} 42 \mathrm{~A}$ & 0.254249 & 0.864854 & 0.110286 & $0.052 *$ & $0.497(3)$ \\
\hline $\mathrm{H} 42 \mathrm{~B}$ & 0.211714 & 0.831318 & 0.183490 & $0.052^{*}$ & $0.497(3)$ \\
\hline $\mathrm{C} 43$ & $0.3112(6)$ & $0.6851(5)$ & $0.1436(3)$ & $0.0511(18)$ & 0.497 (3) \\
\hline $\mathrm{H} 43 \mathrm{~A}$ & 0.383065 & 0.663799 & 0.119040 & $0.061^{*}$ & $0.497(3)$ \\
\hline H43B & 0.329353 & 0.633553 & 0.188787 & $0.061^{*}$ & $0.497(3)$ \\
\hline $\mathrm{C} 44$ & $0.2272(5)$ & $0.6590(7)$ & $0.1066(4)$ & $0.060(2)$ & $0.497(3)$ \\
\hline H44 & 0.150759 & 0.689519 & 0.120651 & $0.072 *$ & $0.497(3)$ \\
\hline C45 & $0.2398(5)$ & $0.5993(6)$ & $0.0561(4)$ & $0.047(2)$ & $0.497(3)$ \\
\hline $\mathrm{C} 46$ & $0.3505(6)$ & $0.5504(6)$ & 0.0255 (4) & $0.066(2)$ & $0.497(3)$ \\
\hline $\mathrm{H} 46 \mathrm{~A}$ & 0.375891 & 0.613372 & 0.006216 & $0.099 *$ & $0.497(3)$ \\
\hline H46B & 0.341025 & 0.510431 & -0.010698 & $0.099^{*}$ & 0.497 (3) \\
\hline $\mathrm{H} 46 \mathrm{C}$ & 0.408322 & 0.494896 & 0.060435 & $0.099 *$ & $0.497(3)$ \\
\hline $\mathrm{C} 47$ & $0.1410(8)$ & $0.5804(10)$ & $0.0233(7)$ & 0.049 (3) & $0.497(3)$ \\
\hline $\mathrm{H} 47 \mathrm{~A}$ & 0.135190 & 0.508335 & 0.047440 & $0.073^{*}$ & $0.497(3)$ \\
\hline H47B & 0.155258 & 0.574342 & -0.024486 & $0.073 *$ & $0.497(3)$ \\
\hline $\mathrm{H} 47 \mathrm{C}$ & 0.068681 & 0.646227 & 0.025625 & $0.073 *$ & $0.497(3)$ \\
\hline $\mathrm{C} 42 \mathrm{~A}$ & 0.2725 (4) & $0.8328(9)$ & $0.1487(6)$ & $0.060(4)$ & $0.234(3)$ \\
\hline $\mathrm{H} 42 \mathrm{C}$ & 0.228484 & 0.909710 & 0.120188 & $0.072 *$ & $0.234(3)$ \\
\hline H42D & 0.219934 & 0.809594 & 0.179676 & $0.072 *$ & $0.234(3)$ \\
\hline C43A & $0.3301(8)$ & $0.7399(9)$ & $0.1038(6)$ & $0.0380(6)$ & $0.234(3)$ \\
\hline $\mathrm{H} 43 \mathrm{C}$ & 0.368236 & 0.774149 & 0.067567 & $0.046^{*}$ & $0.234(3)$ \\
\hline $\mathrm{H} 43 \mathrm{D}$ & 0.391691 & 0.673485 & 0.132496 & $0.046^{*}$ & $0.234(3)$ \\
\hline C44A & $0.2578(11)$ & $0.6938(8)$ & $0.0713(6)$ & 0.049 (3) & $0.234(3)$ \\
\hline $\mathrm{H} 44 \mathrm{~A}$ & 0.204362 & 0.750683 & 0.037274 & $0.058^{*}$ & $0.234(3)$ \\
\hline $\mathrm{C} 45 \mathrm{~A}$ & $0.2486(12)$ & $0.5905(8)$ & $0.0780(10)$ & $0.052(5)$ & $0.234(3)$ \\
\hline $\mathrm{C} 46 \mathrm{~A}$ & $0.3343(12)$ & $0.4821(9)$ & $0.1161(7)$ & $0.057(4)$ & $0.234(3)$ \\
\hline H46D & 0.411449 & 0.484605 & 0.112303 & $0.085^{*}$ & $0.234(3)$ \\
\hline $\mathrm{H} 46 \mathrm{E}$ & 0.332034 & 0.415911 & 0.097398 & $0.085^{*}$ & $0.234(3)$ \\
\hline $\mathrm{H} 46 \mathrm{~F}$ & 0.316724 & 0.473326 & 0.164139 & $0.085^{*}$ & $0.234(3)$ \\
\hline $\mathrm{C} 47 \mathrm{~A}$ & $0.156(2)$ & $0.5714(18)$ & $0.0387(16)$ & $0.054(7)$ & $0.234(3)$ \\
\hline H47D & 0.086551 & 0.587604 & 0.064577 & $0.081^{*}$ & $0.234(3)$ \\
\hline H47E & 0.184113 & 0.490441 & 0.031926 & $0.081^{*}$ & $0.234(3)$ \\
\hline $\mathrm{H} 47 \mathrm{~F}$ & 0.137180 & 0.623773 & -0.005588 & $0.081^{*}$ & $0.234(3)$ \\
\hline $\mathrm{C} 42 \mathrm{~B}$ & 0.2725 (4) & $0.8328(9)$ & $0.1487(6)$ & $0.060(4)$ & $0.269(2)$ \\
\hline $\mathrm{H} 42 \mathrm{E}$ & 0.225387 & 0.913821 & 0.126699 & $0.072 *$ & $0.269(2)$ \\
\hline $\mathrm{H} 42 \mathrm{~F}$ & 0.224383 & 0.807046 & 0.182017 & $0.072 *$ & $0.269(2)$ \\
\hline C43B & $0.2952(9)$ & $0.7563(6)$ & 0.0930 (4) & $0.0380(6)$ & $0.269(2)$ \\
\hline $\mathrm{H} 43 \mathrm{E}$ & 0.224064 & 0.777242 & 0.063822 & $0.046^{*}$ & $0.269(2)$ \\
\hline $\mathrm{H} 43 \mathrm{~F}$ & 0.356195 & 0.766740 & 0.063939 & $0.046^{*}$ & $0.269(2)$ \\
\hline C44B & $0.3320(9)$ & $0.6372(6)$ & $0.1293(5)$ & $0.0467(18)$ & $0.269(2)$ \\
\hline H44B & 0.316213 & 0.621655 & 0.175766 & $0.056^{*}$ & $0.269(2)$ \\
\hline C45B & $0.3881(8)$ & $0.5498(6)$ & $0.0974(5)$ & $0.0467(18)$ & $0.269(2)$ \\
\hline $\mathrm{C} 46 \mathrm{~B}$ & $0.4063(9)$ & $0.4373(7)$ & $0.1404(6)$ & $0.0467(18)$ & $0.269(2)$ \\
\hline H46G & 0.394762 & 0.447379 & 0.187857 & $0.070^{*}$ & $0.269(2)$ \\
\hline $\mathrm{H} 46 \mathrm{H}$ & 0.485119 & 0.382611 & 0.136938 & $0.070^{*}$ & $0.269(2)$ \\
\hline
\end{tabular}




$\begin{array}{llllll}\text { H46I } & 0.351109 & 0.406539 & 0.125334 & 0.070^{*} & 0.269(2) \\ \text { C47B } & 0.4474(11) & 0.5457(10) & 0.0310(5) & 0.069(3) & 0.269(2) \\ \text { H47G } & 0.430097 & 0.625194 & 0.006434 & 0.104^{*} & 0.269(2) \\ \text { H47H } & 0.419473 & 0.503490 & 0.002970 & 0.104^{*} & 0.269(2) \\ \text { H47I } & 0.531009 & 0.505644 & 0.040563 & 0.104^{*} & 0.269(2) \\ \text { O11 } & -0.0152(18) & 0.5008(18) & 0.0211(8) & 0.079(6)^{*} & 0.1346(12) \\ \text { H11 } & -0.014663 & 0.486948 & 0.063744 & 0.119^{*} & 0.1346 \\ \text { C48 } & 0.079(2) & 0.541(2) & 0.0006(10) & 0.059(6)^{*} & 0.1346(12) \\ \text { H48A } & 0.154264 & 0.480177 & 0.019082 & 0.071^{*} & 0.1346(12) \\ \text { H48B } & 0.068535 & 0.612548 & 0.018224 & 0.071^{*} & 0.1346(12) \\ \text { C49 } & 0.074(2) & 0.564(2) & -0.0753(9) & 0.070(7)^{*} & 0.1346(12) \\ \text { H49A } & 0.136285 & 0.589958 & -0.090649 & 0.105^{*} & 0.1346 \\ \text { H49B } & -0.000611 & 0.625148 & -0.092975 & 0.105^{*} & 0.1346 \\ \text { H49C } & 0.083207 & 0.492806 & -0.092152 & 0.105^{*} & 0.1346\end{array}$

Atomic displacement parameters $\left(\AA^{2}\right)$

\begin{tabular}{|c|c|c|c|c|c|c|}
\hline & $U^{11}$ & $U^{22}$ & $U^{33}$ & $U^{12}$ & $U^{13}$ & $U^{23}$ \\
\hline $\mathrm{O} 1$ & $0.0341(10)$ & $0.0472(11)$ & $0.0551(13)$ & $-0.0181(8)$ & $0.0028(9)$ & $-0.0208(10)$ \\
\hline $\mathrm{O} 2$ & $0.0325(9)$ & $0.0461(11)$ & $0.0394(11)$ & $-0.0188(8)$ & $0.0012(8)$ & $-0.0184(9)$ \\
\hline $\mathrm{O} 3$ & $0.0240(8)$ & $0.0278(8)$ & $0.0389(10)$ & $-0.0094(7)$ & $0.0020(7)$ & $-0.0143(8)$ \\
\hline $\mathrm{O} 4$ & $0.0263(9)$ & $0.0346(9)$ & $0.0462(11)$ & $-0.0121(7)$ & $-0.0046(8)$ & $-0.0095(8)$ \\
\hline O5 & $0.0254(8)$ & $0.0276(9)$ & $0.0470(11)$ & $-0.0061(7)$ & $-0.0005(8)$ & $-0.0134(8)$ \\
\hline O6 & $0.0249(8)$ & $0.0351(9)$ & $0.0448(11)$ & $-0.0098(7)$ & $-0.0006(8)$ & $-0.0180(8)$ \\
\hline $\mathrm{C} 1$ & $0.0394(15)$ & $0.0485(17)$ & $0.0477(18)$ & $-0.0208(13)$ & $0.0050(13)$ & $-0.0135(14)$ \\
\hline $\mathrm{C} 2$ & $0.0245(12)$ & $0.0306(12)$ & $0.0317(14)$ & $-0.0119(10)$ & $-0.0025(10)$ & $-0.0059(11)$ \\
\hline $\mathrm{C} 3$ & $0.0345(13)$ & $0.0315(13)$ & $0.0287(14)$ & $-0.0144(11)$ & $-0.0030(11)$ & $-0.0075(11)$ \\
\hline $\mathrm{C} 4$ & $0.0349(14)$ & $0.0421(15)$ & $0.0330(15)$ & $-0.0150(11)$ & $0.0054(12)$ & $-0.0156(12)$ \\
\hline $\mathrm{C} 5$ & $0.0312(13)$ & $0.0382(14)$ & $0.0353(15)$ & $-0.0175(11)$ & $0.0032(11)$ & $-0.0101(12)$ \\
\hline C6 & $0.0301(12)$ & $0.0295(12)$ & $0.0269(13)$ & $-0.0140(10)$ & $-0.0002(10)$ & $-0.0044(10)$ \\
\hline $\mathrm{C} 7$ & $0.0266(12)$ & $0.0290(12)$ & $0.0307(14)$ & $-0.0106(10)$ & $0.0016(10)$ & $-0.0064(11)$ \\
\hline $\mathrm{C} 8$ & $0.0278(12)$ & $0.0309(12)$ & $0.0281(13)$ & $-0.0151(10)$ & $0.0039(10)$ & $-0.0064(10)$ \\
\hline $\mathrm{C} 9$ & $0.0285(12)$ & $0.0288(12)$ & $0.0337(14)$ & $-0.0146(10)$ & $0.0043(11)$ & $-0.0106(11)$ \\
\hline $\mathrm{C} 10$ & $0.0274(12)$ & $0.0266(12)$ & $0.0304(14)$ & $-0.0112(10)$ & $0.0043(10)$ & $-0.0081(10)$ \\
\hline $\mathrm{C} 11$ & $0.0246(12)$ & $0.0290(12)$ & $0.0298(14)$ & $-0.0123(10)$ & $0.0034(10)$ & $-0.0067(10)$ \\
\hline $\mathrm{C} 12$ & $0.0278(13)$ & $0.0298(12)$ & $0.0316(14)$ & $-0.0149(10)$ & $0.0027(11)$ & $-0.0055(11)$ \\
\hline $\mathrm{C} 13$ & $0.0246(12)$ & $0.0267(12)$ & $0.0351(14)$ & $-0.0101(10)$ & $0.0012(10)$ & $-0.0054(11)$ \\
\hline $\mathrm{C} 14$ & $0.0290(12)$ & $0.0257(12)$ & $0.0325(14)$ & $-0.0094(10)$ & $0.0031(11)$ & $-0.0069(11)$ \\
\hline $\mathrm{C} 15$ & $0.0258(13)$ & $0.0349(14)$ & $0.0602(19)$ & $-0.0066(11)$ & $0.0000(12)$ & $-0.0146(13)$ \\
\hline $\mathrm{C} 16$ & $0.0264(12)$ & $0.0312(13)$ & $0.0323(14)$ & $-0.0143(10)$ & $0.0017(10)$ & $-0.0099(11)$ \\
\hline $\mathrm{C} 17$ & $0.0241(11)$ & $0.0290(12)$ & $0.0323(14)$ & $-0.0119(10)$ & $0.0038(10)$ & $-0.0108(11)$ \\
\hline $\mathrm{C} 18$ & $0.0242(12)$ & $0.0327(13)$ & $0.0438(16)$ & $-0.0076(10)$ & $0.0013(11)$ & $-0.0152(12)$ \\
\hline C19 & $0.0251(12)$ & $0.0384(14)$ & $0.0520(18)$ & $-0.0071(11)$ & $-0.0029(12)$ & $-0.0188(13)$ \\
\hline $\mathrm{C} 20$ & $0.0213(12)$ & $0.0396(15)$ & $0.0621(19)$ & $-0.0096(11)$ & $-0.0019(12)$ & $-0.0232(14)$ \\
\hline $\mathrm{C} 21$ & $0.0312(14)$ & $0.0486(17)$ & $0.074(2)$ & $-0.0063(12)$ & $-0.0108(14)$ & $-0.0347(16)$ \\
\hline $\mathrm{C} 22$ & $0.0338(15)$ & $0.0433(16)$ & $0.091(3)$ & $-0.0161(13)$ & $0.0123(16)$ & $-0.0250(17)$ \\
\hline O1A & $0.0471(11)$ & $0.0268(9)$ & $0.0373(11)$ & $-0.0102(8)$ & $-0.0005(8)$ & $-0.0106(8)$ \\
\hline $\mathrm{O} 2 \mathrm{~A}$ & $0.0363(9)$ & $0.0308(9)$ & $0.0354(10)$ & $-0.0109(7)$ & $-0.0004(8)$ & $-0.0133(8)$ \\
\hline
\end{tabular}




\begin{tabular}{|c|c|c|c|c|c|c|}
\hline $\mathrm{O} 3 \mathrm{~A}$ & $0.0265(8)$ & $0.0270(8)$ & $0.0351(10)$ & $-0.0071(7)$ & $-0.0033(7)$ & $-0.0095(7)$ \\
\hline $\mathrm{O} 4 \mathrm{~A}$ & $0.0286(9)$ & $0.0283(9)$ & $0.0459(11)$ & $-0.0040(7)$ & $-0.0004(8)$ & $-0.0142(8)$ \\
\hline $\mathrm{O} 5 \mathrm{~A}$ & $0.0254(8)$ & $0.0349(9)$ & $0.0363(10)$ & $-0.0097(7)$ & $-0.0029(7)$ & $-0.0084(8)$ \\
\hline O6A & $0.0314(9)$ & $0.0247(8)$ & $0.0335(10)$ & $-0.0097(7)$ & $-0.0021(7)$ & $-0.0077(7)$ \\
\hline $\mathrm{C} 1 \mathrm{~A}$ & $0.0416(15)$ & $0.0288(13)$ & $0.0365(15)$ & $-0.0093(11)$ & 0.0007 (12) & $-0.0094(11)$ \\
\hline $\mathrm{C} 2 \mathrm{~A}$ & $0.0292(12)$ & $0.0237(12)$ & $0.0367(15)$ & $-0.0104(10)$ & $0.0038(11)$ & $-0.0070(11)$ \\
\hline $\mathrm{C} 3 \mathrm{~A}$ & $0.0275(12)$ & $0.0318(13)$ & $0.0333(14)$ & $-0.0139(10)$ & $0.0063(11)$ & $-0.0142(11)$ \\
\hline $\mathrm{C} 4 \mathrm{~A}$ & $0.0270(12)$ & $0.0337(13)$ & $0.0315(14)$ & $-0.0098(10)$ & $-0.0004(11)$ & $-0.0092(11)$ \\
\hline $\mathrm{C} 5 \mathrm{~A}$ & $0.0273(12)$ & $0.0278(12)$ & $0.0327(14)$ & $-0.0081(10)$ & $0.0038(11)$ & $-0.0086(11)$ \\
\hline C6A & $0.0264(12)$ & $0.0271(12)$ & $0.0301(14)$ & $-0.0086(10)$ & $0.0014(10)$ & $-0.0099(10)$ \\
\hline C7A & $0.0309(13)$ & $0.0308(13)$ & $0.0311(14)$ & $-0.0115(10)$ & $-0.0006(11)$ & $-0.0052(11)$ \\
\hline $\mathrm{C} 8 \mathrm{~A}$ & $0.0309(12)$ & $0.0247(12)$ & $0.0275(13)$ & $-0.0097(10)$ & $0.0010(10)$ & $-0.0058(10)$ \\
\hline C9A & $0.0300(12)$ & $0.0282(12)$ & 0.0307 (14) & $-0.0114(10)$ & $-0.0028(11)$ & $-0.0073(11)$ \\
\hline $\mathrm{C} 10 \mathrm{~A}$ & $0.0255(12)$ & $0.0244(12)$ & $0.0270(13)$ & $-0.0086(9)$ & $-0.0006(10)$ & $-0.0020(10)$ \\
\hline $\mathrm{C} 11 \mathrm{~A}$ & $0.0267(12)$ & $0.0228(11)$ & $0.0285(13)$ & $-0.0091(9)$ & $0.0037(10)$ & $-0.0055(10)$ \\
\hline $\mathrm{C} 12 \mathrm{~A}$ & $0.0293(12)$ & $0.0245(12)$ & $0.0312(14)$ & $-0.0082(10)$ & 0.0039 (11) & $-0.0043(10)$ \\
\hline C13A & $0.0262(12)$ & $0.0261(12)$ & $0.0303(14)$ & $-0.0088(10)$ & $0.0032(10)$ & $-0.0051(10)$ \\
\hline C14A & $0.0239(12)$ & $0.0301(12)$ & $0.0266(13)$ & $-0.0105(10)$ & $0.0011(10)$ & $-0.0039(10)$ \\
\hline C15A & $0.0268(13)$ & $0.0385(14)$ & $0.0405(16)$ & $-0.0070(11)$ & $-0.0039(11)$ & $-0.0038(12)$ \\
\hline $\mathrm{C} 16 \mathrm{~A}$ & $0.0305(12)$ & $0.0254(12)$ & $0.0255(13)$ & $-0.0122(10)$ & $0.0016(10)$ & $-0.0055(10)$ \\
\hline C17A & $0.0299(12)$ & $0.0229(11)$ & $0.0266(13)$ & $-0.0095(9)$ & $0.0018(10)$ & $-0.0041(10)$ \\
\hline C18A & $0.0269(12)$ & $0.0293(13)$ & $0.0356(15)$ & $-0.0078(10)$ & $-0.0020(11)$ & $-0.0064(11)$ \\
\hline C19A & $0.0327(13)$ & $0.0270(12)$ & $0.0358(15)$ & $-0.0057(10)$ & $-0.0006(11)$ & $-0.0073(11)$ \\
\hline $\mathrm{C} 20 \mathrm{~A}$ & $0.0348(13)$ & $0.0229(12)$ & $0.0346(15)$ & $-0.0078(10)$ & $-0.0008(11)$ & $-0.0067(11)$ \\
\hline $\mathrm{C} 21 \mathrm{~A}$ & $0.0414(15)$ & $0.0296(13)$ & $0.0390(16)$ & $-0.0119(11)$ & $-0.0005(12)$ & $-0.0121(12)$ \\
\hline $\mathrm{C} 22 \mathrm{~A}$ & $0.0438(15)$ & $0.0297(13)$ & $0.0397(16)$ & $-0.0127(11)$ & $0.0025(13)$ & $-0.0055(12)$ \\
\hline $\mathrm{O} 7$ & $0.0334(9)$ & $0.0304(9)$ & $0.0498(12)$ & $-0.0076(7)$ & $-0.0023(9)$ & $-0.0167(9)$ \\
\hline $\mathrm{O} 8$ & $0.0269(9)$ & $0.0322(9)$ & $0.0495(12)$ & $-0.0053(7)$ & $-0.0022(8)$ & $-0.0146(8)$ \\
\hline O9 & $0.0280(9)$ & $0.0296(9)$ & $0.0547(13)$ & $-0.0036(7)$ & $-0.0087(8)$ & $-0.0140(8)$ \\
\hline $\mathrm{C} 23$ & $0.0323(13)$ & $0.0231(12)$ & $0.0297(14)$ & $-0.0103(10)$ & $0.0031(11)$ & $-0.0065(10)$ \\
\hline $\mathrm{C} 24$ & $0.0278(12)$ & $0.0315(13)$ & $0.0377(15)$ & $-0.0140(10)$ & $0.0014(11)$ & $-0.0072(11)$ \\
\hline $\mathrm{C} 25$ & $0.0242(12)$ & $0.0277(12)$ & $0.0348(14)$ & $-0.0091(10)$ & $0.0035(11)$ & $-0.0044(11)$ \\
\hline $\mathrm{C} 26$ & $0.0256(12)$ & $0.0259(12)$ & $0.0287(13)$ & $-0.0086(9)$ & $0.0049(10)$ & $-0.0040(10)$ \\
\hline $\mathrm{C} 27$ & $0.0271(12)$ & $0.0287(12)$ & $0.0329(14)$ & $-0.0080(10)$ & $-0.0020(11)$ & $-0.0070(11)$ \\
\hline $\mathrm{C} 28$ & $0.0274(12)$ & $0.0290(12)$ & $0.0313(14)$ & $-0.0066(10)$ & $-0.0015(11)$ & $-0.0061(11)$ \\
\hline $\mathrm{C} 29$ & $0.0244(12)$ & $0.0274(12)$ & $0.0349(14)$ & $-0.0073(10)$ & $0.0053(11)$ & $-0.0071(11)$ \\
\hline $\mathrm{C} 30$ & $0.0264(12)$ & $0.0269(12)$ & $0.0323(14)$ & $-0.0059(10)$ & $0.0010(11)$ & $-0.0053(11)$ \\
\hline C31 & $0.0260(12)$ & $0.0291(12)$ & $0.0311(14)$ & $-0.0089(10)$ & $0.0039(10)$ & $-0.0064(11)$ \\
\hline $\mathrm{C} 32$ & $0.0254(12)$ & $0.0276(12)$ & $0.0281(13)$ & $-0.0100(10)$ & $0.0050(10)$ & $-0.0066(10)$ \\
\hline $\mathrm{C} 33$ & $0.0247(12)$ & $0.0276(12)$ & $0.0324(14)$ & $-0.0082(10)$ & $0.0015(10)$ & $-0.0050(11)$ \\
\hline C34 & $0.0316(13)$ & $0.0251(12)$ & $0.0286(14)$ & $-0.0098(10)$ & $0.0030(11)$ & $-0.0076(10)$ \\
\hline $\mathrm{C} 35$ & $0.0315(13)$ & $0.0334(13)$ & $0.0233(13)$ & $-0.0139(10)$ & $0.0029(10)$ & $-0.0042(11)$ \\
\hline $\mathrm{C} 36$ & $0.0308(13)$ & $0.0343(13)$ & $0.0304(14)$ & $-0.0047(11)$ & $-0.0015(11)$ & $-0.0064(11)$ \\
\hline $\mathrm{O} 10$ & $0.0333(9)$ & $0.0350(9)$ & $0.0331(10)$ & $-0.0112(7)$ & $-0.0020(8)$ & $-0.0110(8)$ \\
\hline $\mathrm{C} 37$ & $0.0316(13)$ & $0.0294(13)$ & $0.0309(14)$ & $-0.0078(10)$ & $0.0028(11)$ & $-0.0082(11)$ \\
\hline $\mathrm{C} 38$ & $0.0362(13)$ & $0.0317(13)$ & $0.0311(14)$ & $-0.0138(11)$ & $0.0026(11)$ & $-0.0077(11)$ \\
\hline C39 & $0.0389(14)$ & $0.0459(14)$ & $0.0363(14)$ & $-0.0236(12)$ & $0.0034(11)$ & $-0.0089(12)$ \\
\hline C40 & $0.0326(13)$ & $0.0280(13)$ & $0.0374(15)$ & $-0.0060(10)$ & $-0.0002(11)$ & $-0.0081(11)$ \\
\hline
\end{tabular}




\begin{tabular}{lllllll} 
C41 & $0.0530(18)$ & $0.0602(19)$ & $0.0348(16)$ & $-0.0204(15)$ & $0.0053(14)$ & $-0.0107(14)$ \\
C42 & $0.038(5)$ & $0.049(4)$ & $0.038(5)$ & $-0.009(3)$ & $-0.005(4)$ & $-0.013(4)$ \\
C43 & $0.057(4)$ & $0.032(3)$ & $0.068(5)$ & $-0.020(3)$ & $-0.020(3)$ & $-0.009(3)$ \\
C44 & $0.037(3)$ & $0.067(5)$ & $0.086(6)$ & $-0.021(3)$ & $0.003(3)$ & $-0.036(4)$ \\
C45 & $0.038(4)$ & $0.046(4)$ & $0.055(6)$ & $-0.012(3)$ & $-0.003(3)$ & $-0.013(3)$ \\
C46 & $0.070(4)$ & $0.071(5)$ & $0.065(5)$ & $-0.028(4)$ & $0.018(4)$ & $-0.034(4)$ \\
C47 & $0.037(4)$ & $0.053(6)$ & $0.061(8)$ & $-0.018(4)$ & $-0.011(4)$ & $-0.019(5)$ \\
C42A & $0.039(5)$ & $0.091(7)$ & $0.055(7)$ & $-0.019(4)$ & $0.003(4)$ & $-0.041(6)$ \\
C43A & $0.0389(14)$ & $0.0459(14)$ & $0.0363(14)$ & $-0.0236(12)$ & $0.0034(11)$ & $-0.0089(12)$ \\
C44A & $0.083(10)$ & $0.047(6)$ & $0.038(7)$ & $-0.048(7)$ & $0.016(6)$ & $-0.011(6)$ \\
C45A & $0.086(12)$ & $0.045(7)$ & $0.041(11)$ & $-0.043(7)$ & $-0.006(7)$ & $-0.008(7)$ \\
C46A & $0.071(9)$ & $0.058(7)$ & $0.055(9)$ & $-0.042(7)$ & $-0.011(7)$ & $0.000(6)$ \\
C47A & $0.085(15)$ & $0.031(9)$ & $0.045(12)$ & $-0.027(9)$ & $-0.032(9)$ & $0.006(7)$ \\
C42B & $0.039(5)$ & $0.091(7)$ & $0.055(7)$ & $-0.019(4)$ & $0.003(4)$ & $-0.041(6)$ \\
C43B & $0.0389(14)$ & $0.0459(14)$ & $0.0363(14)$ & $-0.0236(12)$ & $0.0034(11)$ & $-0.0089(12)$ \\
C44B & $0.036(3)$ & $0.029(3)$ & $0.073(5)$ & $-0.013(3)$ & $-0.019(3)$ & $-0.003(3)$ \\
C45B & $0.036(3)$ & $0.029(3)$ & $0.073(5)$ & $-0.013(3)$ & $-0.019(3)$ & $-0.003(3)$ \\
C46B & $0.036(3)$ & $0.029(3)$ & $0.073(5)$ & $-0.013(3)$ & $-0.019(3)$ & $-0.003(3)$ \\
C47B & $0.074(9)$ & $0.059(8)$ & $0.078(8)$ & $-0.043(7)$ & $-0.017(6)$ & $0.019(6)$ \\
& & & & & & \\
\hline
\end{tabular}

Geometric parameters $\left(\stackrel{A}{ }{ }^{o}\right)$

\begin{tabular}{llll}
\hline $\mathrm{O} 1-\mathrm{C} 1$ & $1.451(3)$ & $\mathrm{C} 23-\mathrm{C} 28$ & $1.394(3)$ \\
$\mathrm{O} 1-\mathrm{C} 2$ & $1.369(3)$ & $\mathrm{C} 24-\mathrm{H} 24$ & 0.9500 \\
$\mathrm{O} 2-\mathrm{C} 1$ & $1.439(3)$ & $\mathrm{C} 24-\mathrm{C} 25$ & $1.379(3)$ \\
$\mathrm{O} 2-\mathrm{C} 3$ & $1.390(3)$ & $\mathrm{C} 25-\mathrm{H} 25$ & 0.9500 \\
$\mathrm{O} 3-\mathrm{C} 9$ & $\mathrm{C} 25-\mathrm{C} 26$ & $1.397(3)$ \\
$\mathrm{O} 3-\mathrm{C} 10$ & $1.359(3)$ & $\mathrm{C} 26-\mathrm{C} 27$ & $1.405(3)$ \\
$\mathrm{O} 4-\mathrm{C} 12$ & $1.374(3)$ & $\mathrm{C} 26-\mathrm{C} 29$ & $1.457(3)$ \\
$\mathrm{O} 5-\mathrm{C} 14$ & $1.237(3)$ & $\mathrm{C} 27-\mathrm{H} 27$ & 0.9500 \\
$\mathrm{O} 5-\mathrm{C} 15$ & $1.369(3)$ & $\mathrm{C} 27-\mathrm{C} 28$ & $1.381(3)$ \\
$\mathrm{O} 6-\mathrm{C} 16$ & $1.428(3)$ & $\mathrm{C} 28-\mathrm{H} 28$ & 0.9500 \\
$\mathrm{O} 6-\mathrm{C} 20$ & $1.356(3)$ & $\mathrm{C} 29-\mathrm{H} 29$ & 0.9500 \\
$\mathrm{C} 1-\mathrm{H} 1 \mathrm{~A}$ & $1.475(3)$ & $\mathrm{C} 29-\mathrm{C} 30$ & $1.341(3)$ \\
$\mathrm{C} 1-\mathrm{H} 1 \mathrm{~B}$ & 0.9900 & $\mathrm{C} 30-\mathrm{H} 30$ & 0.9500 \\
$\mathrm{C} 2-\mathrm{C} 3$ & $\mathrm{C} 30-\mathrm{C} 31$ & $1.463(3)$ \\
$\mathrm{C} 2-\mathrm{C} 7$ & 1.9900 & $\mathrm{C} 31-\mathrm{C} 32$ & $1.459(3)$ \\
$\mathrm{C} 3-\mathrm{C} 4$ & $1.384(3)$ & $\mathrm{C} 32-\mathrm{C} 33$ & $1.409(3)$ \\
$\mathrm{C} 4-\mathrm{H} 4$ & $\mathrm{C} 32-\mathrm{C} 37$ & $1.407(3)$ \\
$\mathrm{C} 4-\mathrm{C} 5$ & $1.367(3)$ & $\mathrm{C} 33-\mathrm{C} 34$ & $1.391(3)$ \\
$\mathrm{C} 5-\mathrm{H} 5$ & $\mathrm{C} 34-\mathrm{H} 34$ & 0.9500 \\
$\mathrm{C} 5-\mathrm{C} 6$ & $\mathrm{C} 34-\mathrm{C} 35$ & $1.379(3)$ \\
$\mathrm{C} 6-\mathrm{C} 7$ & $1.400(4)$ & $\mathrm{C} 35-\mathrm{C} 36$ & $1.399(3)$ \\
$\mathrm{C} 6-\mathrm{C} 8$ & 0.9500 & $\mathrm{C} 35-\mathrm{O} 10$ & $1.363(3)$ \\
$\mathrm{C} 7-\mathrm{H} 7 \mathrm{~A}$ & $1.399(3)$ & $\mathrm{C} 36-\mathrm{H} 36$ & 0.9500 \\
$\mathrm{C} 8-\mathrm{C} 9$ & $1.407(3)$ & $\mathrm{C} 36-\mathrm{C} 37$ & $1.367(3)$ \\
$\mathrm{C} 8-\mathrm{C} 12$ & $1.481(3)$ & $\mathrm{O} 10-\mathrm{C} 38$ & $1.449(3)$ \\
$\mathrm{C} 9-\mathrm{H} 9 \mathrm{~A}$ & 0.9500 & $\mathrm{C} 37-\mathrm{H} 37$ & 0.9500
\end{tabular}




\begin{tabular}{|c|c|c|c|}
\hline $\mathrm{C} 10-\mathrm{C} 11$ & $1.388(3)$ & $\mathrm{C} 38-\mathrm{H} 38 \mathrm{~A}$ & 0.9900 \\
\hline $\mathrm{C} 10-\mathrm{C} 17$ & $1.393(3)$ & C $38-\mathrm{H} 38 \mathrm{~B}$ & 0.9900 \\
\hline $\mathrm{C} 11-\mathrm{C} 12$ & $1.464(3)$ & C38-C39 & $1.494(4)$ \\
\hline $\mathrm{C} 11-\mathrm{C} 13$ & $1.412(3)$ & C39-H39 & 0.9500 \\
\hline $\mathrm{C} 13-\mathrm{H} 13$ & 0.9500 & $\mathrm{C} 39-\mathrm{C} 40$ & $1.331(3)$ \\
\hline $\mathrm{C} 13-\mathrm{C} 14$ & $1.374(3)$ & $\mathrm{C} 40-\mathrm{C} 41$ & $1.491(4)$ \\
\hline $\mathrm{C} 14-\mathrm{C} 16$ & $1.416(3)$ & $\mathrm{C} 40-\mathrm{C} 42$ & $1.510(4)$ \\
\hline C15-H15A & 0.9800 & $\mathrm{C} 40-\mathrm{C} 42 \mathrm{~A}$ & $1.510(4)$ \\
\hline C15-H15B & 0.9800 & $\mathrm{C} 40-\mathrm{C} 42 \mathrm{~B}$ & $1.510(4)$ \\
\hline $\mathrm{C} 15-\mathrm{H} 15 \mathrm{C}$ & 0.9800 & $\mathrm{C} 41-\mathrm{H} 41 \mathrm{~A}$ & 0.9800 \\
\hline $\mathrm{C} 16-\mathrm{C} 17$ & $1.389(3)$ & $\mathrm{C} 41-\mathrm{H} 41 \mathrm{~B}$ & 0.9800 \\
\hline $\mathrm{C} 17-\mathrm{C} 18$ & $1.456(3)$ & $\mathrm{C} 41-\mathrm{H} 41 \mathrm{C}$ & 0.9800 \\
\hline C18-H18 & 0.9500 & $\mathrm{C} 42-\mathrm{H} 42 \mathrm{~A}$ & 0.9900 \\
\hline $\mathrm{C} 18-\mathrm{C} 19$ & $1.326(4)$ & $\mathrm{C} 42-\mathrm{H} 42 \mathrm{~B}$ & 0.9900 \\
\hline C19-H19 & 0.9500 & $\mathrm{C} 42-\mathrm{C} 43$ & $1.551(6)$ \\
\hline $\mathrm{C} 19-\mathrm{C} 20$ & $1.504(3)$ & $\mathrm{C} 42-\mathrm{C} 43 \mathrm{~B}$ & $1.500(10)$ \\
\hline $\mathrm{C} 20-\mathrm{C} 21$ & $1.518(4)$ & $\mathrm{C} 43-\mathrm{H} 43 \mathrm{~A}$ & 0.9900 \\
\hline $\mathrm{C} 20-\mathrm{C} 22$ & $1.515(4)$ & $\mathrm{C} 43-\mathrm{H} 43 \mathrm{~B}$ & 0.9900 \\
\hline $\mathrm{C} 21-\mathrm{H} 21 \mathrm{~A}$ & 0.9800 & $\mathrm{C} 43-\mathrm{C} 44$ & $1.453(6)$ \\
\hline $\mathrm{C} 21-\mathrm{H} 21 \mathrm{~B}$ & 0.9800 & $\mathrm{C} 43-\mathrm{C} 42 \mathrm{~B}$ & $1.759(11)$ \\
\hline $\mathrm{C} 21-\mathrm{H} 21 \mathrm{C}$ & 0.9800 & $\mathrm{C} 43-\mathrm{C} 43 \mathrm{~B}$ & $1.192(11)$ \\
\hline $\mathrm{C} 22-\mathrm{H} 22 \mathrm{~A}$ & 0.9800 & $\mathrm{C} 43-\mathrm{C} 44 \mathrm{~B}$ & $0.673(8)$ \\
\hline $\mathrm{C} 22-\mathrm{H} 22 \mathrm{~B}$ & 0.9800 & $\mathrm{C} 43-\mathrm{C} 45 \mathrm{~B}$ & $1.996(9)$ \\
\hline $\mathrm{C} 22-\mathrm{H} 22 \mathrm{C}$ & 0.9800 & C44-H44 & 0.9500 \\
\hline $\mathrm{O} 1 \mathrm{~A}-\mathrm{C} 1 \mathrm{~A}$ & $1.441(3)$ & $\mathrm{C} 44-\mathrm{C} 45$ & $1.332(4)$ \\
\hline $\mathrm{O} 1 \mathrm{~A}-\mathrm{C} 2 \mathrm{~A}$ & $1.382(3)$ & $\mathrm{C} 44-\mathrm{C} 43 \mathrm{~B}$ & $1.722(12)$ \\
\hline $\mathrm{O} 2 \mathrm{~A}-\mathrm{C} 1 \mathrm{~A}$ & $1.429(3)$ & C44-C44B & $1.303(13)$ \\
\hline $\mathrm{O} 2 \mathrm{~A}-\mathrm{C} 3 \mathrm{~A}$ & $1.380(3)$ & $\mathrm{C} 44-\mathrm{C} 45 \mathrm{~B}$ & $1.997(10)$ \\
\hline $\mathrm{O} 3 \mathrm{~A}-\mathrm{C} 9 \mathrm{~A}$ & $1.357(3)$ & $\mathrm{C} 45-\mathrm{C} 46$ & $1.468(8)$ \\
\hline $\mathrm{O} 3 \mathrm{~A}-\mathrm{C} 10 \mathrm{~A}$ & $1.374(3)$ & $\mathrm{C} 45-\mathrm{C} 47$ & $1.518(7)$ \\
\hline $\mathrm{O} 4 \mathrm{~A}-\mathrm{C} 12 \mathrm{~A}$ & $1.248(3)$ & $\mathrm{C} 45-\mathrm{C} 45 \mathrm{~B}$ & $1.867(11)$ \\
\hline $\mathrm{O} 5 \mathrm{~A}-\mathrm{C} 14 \mathrm{~A}$ & $1.363(3)$ & $\mathrm{C} 46-\mathrm{H} 46 \mathrm{~A}$ & 0.9800 \\
\hline $\mathrm{O} 5 \mathrm{~A}-\mathrm{C} 15 \mathrm{~A}$ & $1.435(3)$ & $\mathrm{C} 46-\mathrm{H} 46 \mathrm{~B}$ & 0.9800 \\
\hline $\mathrm{O} 6 \mathrm{~A}-\mathrm{C} 16 \mathrm{~A}$ & $1.356(3)$ & $\mathrm{C} 46-\mathrm{H} 46 \mathrm{C}$ & 0.9800 \\
\hline O6A-C20A & $1.473(3)$ & $\mathrm{C} 46-\mathrm{C} 45 \mathrm{~B}$ & $1.511(12)$ \\
\hline $\mathrm{C} 1 \mathrm{~A}-\mathrm{H} 1 \mathrm{AA}$ & 0.9900 & $\mathrm{C} 46-\mathrm{C} 47 \mathrm{~B}$ & $1.197(13)$ \\
\hline $\mathrm{C} 1 \mathrm{~A}-\mathrm{H} 1 \mathrm{AB}$ & 0.9900 & $\mathrm{C} 47-\mathrm{H} 47 \mathrm{~A}$ & 0.9800 \\
\hline $\mathrm{C} 2 \mathrm{~A}-\mathrm{C} 3 \mathrm{~A}$ & $1.377(3)$ & C47-H47B & 0.9800 \\
\hline $\mathrm{C} 2 \mathrm{~A}-\mathrm{C} 7 \mathrm{~A}$ & $1.371(3)$ & $\mathrm{C} 47-\mathrm{H} 47 \mathrm{C}$ & 0.9800 \\
\hline $\mathrm{C} 3 \mathrm{~A}-\mathrm{C} 4 \mathrm{~A}$ & $1.364(3)$ & $\mathrm{C} 47-\mathrm{C} 48$ & $1.20(3)$ \\
\hline $\mathrm{C} 4 \mathrm{~A}-\mathrm{H} 4 \mathrm{~A}$ & 0.9500 & $\mathrm{C} 42 \mathrm{~A}-\mathrm{H} 42 \mathrm{C}$ & 0.9900 \\
\hline $\mathrm{C} 4 \mathrm{~A}-\mathrm{C} 5 \mathrm{~A}$ & $1.402(3)$ & $\mathrm{C} 42 \mathrm{~A}-\mathrm{H} 42 \mathrm{D}$ & 0.9900 \\
\hline C5A-H5A & 0.9500 & $\mathrm{C} 42 \mathrm{~A}-\mathrm{C} 43 \mathrm{~A}$ & $1.554(6)$ \\
\hline $\mathrm{C} 5 \mathrm{~A}-\mathrm{C} 6 \mathrm{~A}$ & $1.390(3)$ & $\mathrm{C} 43 \mathrm{~A}-\mathrm{H} 43 \mathrm{C}$ & 0.9900 \\
\hline $\mathrm{C} 6 \mathrm{~A}-\mathrm{C} 7 \mathrm{~A}$ & $1.414(3)$ & $\mathrm{C} 43 \mathrm{~A}-\mathrm{H} 43 \mathrm{D}$ & 0.9900 \\
\hline $\mathrm{C} 6 \mathrm{~A}-\mathrm{C} 8 \mathrm{~A}$ & $1.486(3)$ & $\mathrm{C} 43 \mathrm{~A}-\mathrm{C} 44 \mathrm{~A}$ & $1.452(6)$ \\
\hline C7A-H7AA & 0.9500 & $\mathrm{C} 44 \mathrm{~A}-\mathrm{H} 44 \mathrm{~A}$ & 0.9500 \\
\hline C8A-C9A & $1.351(3)$ & $\mathrm{C} 44 \mathrm{~A}-\mathrm{C} 45 \mathrm{~A}$ & $1.332(4)$ \\
\hline
\end{tabular}




\begin{tabular}{|c|c|c|c|}
\hline $\mathrm{C} 8 \mathrm{~A}-\mathrm{C} 12 \mathrm{~A}$ & $1.458(3)$ & $\mathrm{C} 45 \mathrm{~A}-\mathrm{C} 46 \mathrm{~A}$ & $1.467(8)$ \\
\hline C9A-H9AA & 0.9500 & $\mathrm{C} 45 \mathrm{~A}-\mathrm{C} 47 \mathrm{~A}$ & $1.518(7)$ \\
\hline $\mathrm{C} 10 \mathrm{~A}-\mathrm{C} 11 \mathrm{~A}$ & $1.389(3)$ & $\mathrm{C} 46 \mathrm{~A}-\mathrm{H} 46 \mathrm{D}$ & 0.9800 \\
\hline $\mathrm{C} 10 \mathrm{~A}-\mathrm{C} 17 \mathrm{~A}$ & $1.403(3)$ & $\mathrm{C} 46 \mathrm{~A}-\mathrm{H} 46 \mathrm{E}$ & 0.9800 \\
\hline $\mathrm{C} 11 \mathrm{~A}-\mathrm{C} 12 \mathrm{~A}$ & $1.450(3)$ & $\mathrm{C} 46 \mathrm{~A}-\mathrm{H} 46 \mathrm{~F}$ & 0.9800 \\
\hline $\mathrm{C} 11 \mathrm{~A}-\mathrm{C} 13 \mathrm{~A}$ & $1.406(3)$ & $\mathrm{C} 47 \mathrm{~A}-\mathrm{H} 47 \mathrm{D}$ & 0.9800 \\
\hline $\mathrm{C} 13 \mathrm{~A}-\mathrm{H} 13 \mathrm{~A}$ & 0.9500 & $\mathrm{C} 47 \mathrm{~A}-\mathrm{H} 47 \mathrm{E}$ & 0.9800 \\
\hline $\mathrm{C} 13 \mathrm{~A}-\mathrm{C} 14 \mathrm{~A}$ & $1.369(3)$ & $\mathrm{C} 47 \mathrm{~A}-\mathrm{H} 47 \mathrm{~F}$ & 0.9800 \\
\hline $\mathrm{C} 14 \mathrm{~A}-\mathrm{C} 16 \mathrm{~A}$ & $1.417(3)$ & $\mathrm{C} 42 \mathrm{~B}-\mathrm{H} 42 \mathrm{E}$ & 0.9900 \\
\hline $\mathrm{C} 15 \mathrm{~A}-\mathrm{H} 15 \mathrm{D}$ & 0.9800 & $\mathrm{C} 42 \mathrm{~B}-\mathrm{H} 42 \mathrm{~F}$ & 0.9900 \\
\hline $\mathrm{C} 15 \mathrm{~A}-\mathrm{H} 15 \mathrm{E}$ & 0.9800 & $\mathrm{C} 42 \mathrm{~B}-\mathrm{C} 43 \mathrm{~B}$ & $1.551(6)$ \\
\hline $\mathrm{C} 15 \mathrm{~A}-\mathrm{H} 15 \mathrm{~F}$ & 0.9800 & $\mathrm{C} 43 \mathrm{~B}-\mathrm{H} 43 \mathrm{E}$ & 0.9900 \\
\hline $\mathrm{C} 16 \mathrm{~A}-\mathrm{C} 17 \mathrm{~A}$ & $1.384(3)$ & $\mathrm{C} 43 \mathrm{~B}-\mathrm{H} 43 \mathrm{~F}$ & 0.9900 \\
\hline $\mathrm{C} 17 \mathrm{~A}-\mathrm{C} 18 \mathrm{~A}$ & $1.461(3)$ & $\mathrm{C} 43 \mathrm{~B}-\mathrm{C} 44 \mathrm{~B}$ & $1.450(6)$ \\
\hline $\mathrm{C} 18 \mathrm{~A}-\mathrm{H} 18 \mathrm{~A}$ & 0.9500 & $\mathrm{C} 44 \mathrm{~B}-\mathrm{H} 44 \mathrm{~B}$ & 0.9500 \\
\hline $\mathrm{C} 18 \mathrm{~A}-\mathrm{C} 19 \mathrm{~A}$ & $1.330(3)$ & $\mathrm{C} 44 \mathrm{~B}-\mathrm{C} 45 \mathrm{~B}$ & $1.330(4)$ \\
\hline C19A-H19A & 0.9500 & $\mathrm{C} 45 \mathrm{~B}-\mathrm{C} 46 \mathrm{~B}$ & $1.464(8)$ \\
\hline $\mathrm{C} 19 \mathrm{~A}-\mathrm{C} 20 \mathrm{~A}$ & $1.502(4)$ & $\mathrm{C} 45 \mathrm{~B}-\mathrm{C} 47 \mathrm{~B}$ & $1.516(7)$ \\
\hline $\mathrm{C} 20 \mathrm{~A}-\mathrm{C} 21 \mathrm{~A}$ & $1.517(3)$ & $\mathrm{C} 46 \mathrm{~B}-\mathrm{H} 46 \mathrm{G}$ & 0.9800 \\
\hline $\mathrm{C} 20 \mathrm{~A}-\mathrm{C} 22 \mathrm{~A}$ & $1.529(4)$ & $\mathrm{C} 46 \mathrm{~B}-\mathrm{H} 46 \mathrm{H}$ & 0.9800 \\
\hline $\mathrm{C} 21 \mathrm{~A}-\mathrm{H} 21 \mathrm{D}$ & 0.9800 & $\mathrm{C} 46 \mathrm{~B}-\mathrm{H} 46 \mathrm{I}$ & 0.9800 \\
\hline $\mathrm{C} 21 \mathrm{~A}-\mathrm{H} 21 \mathrm{E}$ & 0.9800 & $\mathrm{C} 47 \mathrm{~B}-\mathrm{H} 47 \mathrm{G}$ & 0.9800 \\
\hline $\mathrm{C} 21 \mathrm{~A}-\mathrm{H} 21 \mathrm{~F}$ & 0.9800 & $\mathrm{C} 47 \mathrm{~B}-\mathrm{H} 47 \mathrm{H}$ & 0.9800 \\
\hline $\mathrm{C} 22 \mathrm{~A}-\mathrm{H} 22 \mathrm{D}$ & 0.9800 & $\mathrm{C} 47 \mathrm{~B}-\mathrm{H} 47 \mathrm{I}$ & 0.9800 \\
\hline $\mathrm{C} 22 \mathrm{~A}-\mathrm{H} 22 \mathrm{E}$ & 0.9800 & $\mathrm{O} 11-\mathrm{H} 11$ & 0.8400 \\
\hline $\mathrm{C} 22 \mathrm{~A}-\mathrm{H} 22 \mathrm{~F}$ & 0.9800 & $\mathrm{O} 11-\mathrm{C} 48$ & $1.47(3)$ \\
\hline $\mathrm{O} 7-\mathrm{H} 7$ & 0.8400 & $\mathrm{C} 48-\mathrm{H} 48 \mathrm{~A}$ & 0.9900 \\
\hline $\mathrm{O} 7-\mathrm{C} 23$ & $1.361(3)$ & $\mathrm{C} 48-\mathrm{H} 48 \mathrm{~B}$ & 0.9900 \\
\hline $\mathrm{O} 8-\mathrm{C} 31$ & $1.257(3)$ & $\mathrm{C} 48-\mathrm{C} 49$ & $1.491(17)$ \\
\hline O9- & 0.8400 & $\mathrm{C} 49-\mathrm{H} 49 \mathrm{~A}$ & 0.9800 \\
\hline $\mathrm{O} 9-\mathrm{C} 33$ & $1.350(3)$ & $\mathrm{C} 49-\mathrm{H} 49 \mathrm{~B}$ & 0.9800 \\
\hline $\mathrm{C} 23-\mathrm{C} 24$ & $1.391(3)$ & $\mathrm{C} 49-\mathrm{H} 49 \mathrm{C}$ & 0.9800 \\
\hline $\mathrm{C} 2-\mathrm{O} 1-\mathrm{C} 1$ & $104.48(18)$ & $\mathrm{C} 38-\mathrm{C} 39-\mathrm{H} 39$ & 116.6 \\
\hline $\mathrm{C} 3-\mathrm{O} 2-\mathrm{C} 1$ & $102.90(19)$ & $\mathrm{C} 40-\mathrm{C} 39-\mathrm{C} 38$ & $126.7(3)$ \\
\hline $\mathrm{C} 9-\mathrm{O} 3-\mathrm{C} 10$ & $118.00(18)$ & $\mathrm{C} 40-\mathrm{C} 39-\mathrm{H} 39$ & 116.6 \\
\hline $\mathrm{C} 14-\mathrm{O} 5-\mathrm{C} 15$ & $116.40(19)$ & $\mathrm{C} 39-\mathrm{C} 40-\mathrm{C} 41$ & $124.6(3)$ \\
\hline $\mathrm{C} 16-\mathrm{O} 6-\mathrm{C} 20$ & $118.84(18)$ & $\mathrm{C} 39-\mathrm{C} 40-\mathrm{C} 42$ & $118.6(5)$ \\
\hline $\mathrm{O} 1-\mathrm{C} 1-\mathrm{H} 1 \mathrm{~A}$ & 110.5 & $\mathrm{C} 39-\mathrm{C} 40-\mathrm{C} 42 \mathrm{~A}$ & $122.5(5)$ \\
\hline $\mathrm{O} 1-\mathrm{C} 1-\mathrm{H} 1 \mathrm{~B}$ & 110.5 & $\mathrm{C} 39-\mathrm{C} 40-\mathrm{C} 42 \mathrm{~B}$ & $122.5(5)$ \\
\hline $\mathrm{O} 2-\mathrm{C} 1-\mathrm{O} 1$ & $106.3(2)$ & $\mathrm{C} 41-\mathrm{C} 40-\mathrm{C} 42$ & $116.4(5)$ \\
\hline $\mathrm{O} 2-\mathrm{C} 1-\mathrm{H} 1 \mathrm{~A}$ & 110.5 & $\mathrm{C} 41-\mathrm{C} 40-\mathrm{C} 42 \mathrm{~A}$ & $112.9(5)$ \\
\hline $\mathrm{O} 2-\mathrm{C} 1-\mathrm{H} 1 \mathrm{~B}$ & 110.5 & $\mathrm{C} 41-\mathrm{C} 40-\mathrm{C} 42 \mathrm{~B}$ & $112.9(5)$ \\
\hline $\mathrm{H} 1 \mathrm{~A}-\mathrm{C} 1-\mathrm{H} 1 \mathrm{~B}$ & 108.7 & $\mathrm{C} 42 \mathrm{~B}-\mathrm{C} 40-\mathrm{C} 42$ & $9.6(7)$ \\
\hline $\mathrm{O} 1-\mathrm{C} 2-\mathrm{C} 3$ & $108.6(2)$ & $\mathrm{C} 40-\mathrm{C} 41-\mathrm{H} 41 \mathrm{~A}$ & 109.5 \\
\hline $\mathrm{C} 7-\mathrm{C} 2-\mathrm{O} 1$ & $129.0(2)$ & $\mathrm{C} 40-\mathrm{C} 41-\mathrm{H} 41 \mathrm{~B}$ & 109.5 \\
\hline $\mathrm{C} 7-\mathrm{C} 2-\mathrm{C} 3$ & $122.4(2)$ & $\mathrm{C} 40-\mathrm{C} 41-\mathrm{H} 41 \mathrm{C}$ & 109.5 \\
\hline $\mathrm{C} 2-\mathrm{C} 3-\mathrm{O} 2$ & $110.7(2)$ & $\mathrm{H} 41 \mathrm{~A}-\mathrm{C} 41-\mathrm{H} 41 \mathrm{~B}$ & 109.5 \\
\hline
\end{tabular}




\begin{tabular}{|c|c|}
\hline $\mathrm{C} 4-\mathrm{C} 3-\mathrm{O} 2$ & $127.3(2)$ \\
\hline $\mathrm{C} 4-\mathrm{C} 3-\mathrm{C} 2$ & $121.9(2)$ \\
\hline $\mathrm{C} 3-\mathrm{C} 4-\mathrm{H} 4$ & 121.8 \\
\hline $\mathrm{C} 3-\mathrm{C} 4-\mathrm{C} 5$ & $116.5(2)$ \\
\hline $\mathrm{C} 5-\mathrm{C} 4-\mathrm{H} 4$ & 121.8 \\
\hline $\mathrm{C} 4-\mathrm{C} 5-\mathrm{H} 5$ & 118.8 \\
\hline $\mathrm{C} 6-\mathrm{C} 5-\mathrm{C} 4$ & $122.4(2)$ \\
\hline $\mathrm{C} 6-\mathrm{C} 5-\mathrm{H} 5$ & 118.8 \\
\hline $\mathrm{C} 5-\mathrm{C} 6-\mathrm{C} 7$ & $119.3(2)$ \\
\hline $\mathrm{C} 5-\mathrm{C} 6-\mathrm{C} 8$ & $120.0(2)$ \\
\hline $\mathrm{C} 7-\mathrm{C} 6-\mathrm{C} 8$ & $120.5(2)$ \\
\hline $\mathrm{C} 2-\mathrm{C} 7-\mathrm{C} 6$ & $117.4(2)$ \\
\hline $\mathrm{C} 2-\mathrm{C} 7-\mathrm{H} 7 \mathrm{~A}$ & 121.3 \\
\hline $\mathrm{C} 6-\mathrm{C} 7-\mathrm{H} 7 \mathrm{~A}$ & 121.3 \\
\hline $\mathrm{C} 9-\mathrm{C} 8-\mathrm{C} 6$ & $118.2(2)$ \\
\hline $\mathrm{C} 9-\mathrm{C} 8-\mathrm{C} 12$ & $118.7(2)$ \\
\hline $\mathrm{C} 12-\mathrm{C} 8-\mathrm{C} 6$ & $123.1(2)$ \\
\hline $\mathrm{O} 3-\mathrm{C} 9-\mathrm{H} 9 \mathrm{~A}$ & 117.0 \\
\hline $\mathrm{C} 8-\mathrm{C} 9-\mathrm{O} 3$ & $126.1(2)$ \\
\hline $\mathrm{C} 8-\mathrm{C} 9-\mathrm{H} 9 \mathrm{~A}$ & 117.0 \\
\hline $\mathrm{O} 3-\mathrm{C} 10-\mathrm{C} 11$ & $121.4(2)$ \\
\hline $\mathrm{O} 3-\mathrm{C} 10-\mathrm{C} 17$ & $115.6(2)$ \\
\hline $\mathrm{C} 11-\mathrm{C} 10-\mathrm{C} 17$ & $123.0(2)$ \\
\hline $\mathrm{C} 10-\mathrm{C} 11-\mathrm{C} 12$ & $121.0(2)$ \\
\hline $\mathrm{C} 10-\mathrm{C} 11-\mathrm{C} 13$ & $117.8(2)$ \\
\hline $\mathrm{C} 13-\mathrm{C} 11-\mathrm{C} 12$ & $121.2(2)$ \\
\hline $\mathrm{O} 4-\mathrm{C} 12-\mathrm{C} 8$ & $123.3(2)$ \\
\hline $\mathrm{O} 4-\mathrm{C} 12-\mathrm{C} 11$ & $122.0(2)$ \\
\hline $\mathrm{C} 11-\mathrm{C} 12-\mathrm{C} 8$ & $114.7(2)$ \\
\hline $\mathrm{C} 11-\mathrm{C} 13-\mathrm{H} 13$ & 119.6 \\
\hline $\mathrm{C} 14-\mathrm{C} 13-\mathrm{C} 11$ & $120.9(2)$ \\
\hline $\mathrm{C} 14-\mathrm{C} 13-\mathrm{H} 13$ & 119.6 \\
\hline $\mathrm{O} 5-\mathrm{C} 14-\mathrm{C} 13$ & $125.4(2)$ \\
\hline $\mathrm{O} 5-\mathrm{C} 14-\mathrm{C} 16$ & $115.0(2)$ \\
\hline $\mathrm{C} 13-\mathrm{C} 14-\mathrm{C} 16$ & $119.6(2)$ \\
\hline $\mathrm{O} 5-\mathrm{C} 15-\mathrm{H} 15 \mathrm{~A}$ & 109.5 \\
\hline $\mathrm{O} 5-\mathrm{C} 15-\mathrm{H} 15 \mathrm{~B}$ & 109.5 \\
\hline $\mathrm{O} 5-\mathrm{C} 15-\mathrm{H} 15 \mathrm{C}$ & 109.5 \\
\hline $\mathrm{H} 15 \mathrm{~A}-\mathrm{C} 15-\mathrm{H} 15 \mathrm{~B}$ & 109.5 \\
\hline $\mathrm{H} 15 \mathrm{~A}-\mathrm{C} 15-\mathrm{H} 15 \mathrm{C}$ & 109.5 \\
\hline $\mathrm{H} 15 \mathrm{~B}-\mathrm{C} 15-\mathrm{H} 15 \mathrm{C}$ & 109.5 \\
\hline $\mathrm{O} 6-\mathrm{C} 16-\mathrm{C} 14$ & $117.0(2)$ \\
\hline $\mathrm{O} 6-\mathrm{C} 16-\mathrm{C} 17$ & $122.1(2)$ \\
\hline $\mathrm{C} 17-\mathrm{C} 16-\mathrm{C} 14$ & $120.7(2)$ \\
\hline $\mathrm{C} 10-\mathrm{C} 17-\mathrm{C} 18$ & $123.8(2)$ \\
\hline $\mathrm{C} 16-\mathrm{C} 17-\mathrm{C} 10$ & $117.9(2)$ \\
\hline $\mathrm{C} 16-\mathrm{C} 17-\mathrm{C} 18$ & $118.2(2)$ \\
\hline $\mathrm{C} 17-\mathrm{C} 18-\mathrm{H} 18$ & 120.3 \\
\hline
\end{tabular}

$\mathrm{H} 41 \mathrm{~A}-\mathrm{C} 41-\mathrm{H} 41 \mathrm{C} \quad 109.5$

$\mathrm{H} 41 \mathrm{~B}-\mathrm{C} 41-\mathrm{H} 41 \mathrm{C} \quad 109.5$

$\mathrm{C} 40-\mathrm{C} 42-\mathrm{H} 42 \mathrm{~A}$

$\mathrm{C} 40-\mathrm{C} 42-\mathrm{H} 42 \mathrm{~B} \quad 108.4$

$\mathrm{C} 40-\mathrm{C} 42-\mathrm{C} 43 \quad 115.5$ (4)

$\mathrm{H} 42 \mathrm{~A}-\mathrm{C} 42-\mathrm{H} 42 \mathrm{~B} \quad 107.5$

$\mathrm{C} 43-\mathrm{C} 42-\mathrm{H} 42 \mathrm{~A}$

$\mathrm{C} 43-\mathrm{C} 42-\mathrm{H} 42 \mathrm{~B} \quad 108.4$

$\mathrm{C} 43 \mathrm{~B}-\mathrm{C} 42-\mathrm{C} 40 \quad 122.8(7)$

$\mathrm{C} 43 \mathrm{~B}-\mathrm{C} 42-\mathrm{H} 42 \mathrm{~A} \quad 63.1$

$\mathrm{C} 43 \mathrm{~B}-\mathrm{C} 42-\mathrm{H} 42 \mathrm{~B} \quad 128.5$

$\mathrm{C} 43 \mathrm{~B}-\mathrm{C} 42-\mathrm{C} 43 \quad 46.0$ (5)

$\mathrm{C} 42-\mathrm{C} 43-\mathrm{H} 43 \mathrm{~A} \quad 107.8$

$\mathrm{C} 42-\mathrm{C} 43-\mathrm{H} 43 \mathrm{~B} \quad 107.8$

$\mathrm{C} 42-\mathrm{C} 43-\mathrm{C} 42 \mathrm{~B} \quad 5.0(7)$

$\mathrm{C} 42-\mathrm{C} 43-\mathrm{C} 45 \mathrm{~B} \quad 154.9$ (7)

$\mathrm{H} 43 \mathrm{~A}-\mathrm{C} 43-\mathrm{H} 43 \mathrm{~B} \quad 107.1$

$\mathrm{C} 44-\mathrm{C} 43-\mathrm{C} 42 \quad 118.1$ (5)

$\mathrm{C} 44-\mathrm{C} 43-\mathrm{H} 43 \mathrm{~A} \quad 107.8$

$\mathrm{C} 44-\mathrm{C} 43-\mathrm{H} 43 \mathrm{~B} \quad 107.8$

$\mathrm{C} 44-\mathrm{C} 43-\mathrm{C} 42 \mathrm{~B} \quad 115.2(5)$

$\mathrm{C} 44-\mathrm{C} 43-\mathrm{C} 45 \mathrm{~B} \quad 68.7$ (4)

$\mathrm{C} 42 \mathrm{~B}-\mathrm{C} 43-\mathrm{H} 43 \mathrm{~A} \quad 105.7$

$\mathrm{C} 42 \mathrm{~B}-\mathrm{C} 43-\mathrm{H} 43 \mathrm{~B} \quad 112.8$

$\mathrm{C} 42 \mathrm{~B}-\mathrm{C} 43-\mathrm{C} 45 \mathrm{~B} \quad 151.0(7)$

$\mathrm{C} 43 \mathrm{~B}-\mathrm{C} 43-\mathrm{C} 42 \quad 64.8(6)$

$\mathrm{C} 43 \mathrm{~B}-\mathrm{C} 43-\mathrm{H} 43 \mathrm{~A} \quad 72.1$

C43B-C43-H43B $\quad 171.1$

$\mathrm{C} 43 \mathrm{~B}-\mathrm{C} 43-\mathrm{C} 44 \quad 80.6(6)$

$\mathrm{C} 43 \mathrm{~B}-\mathrm{C} 43-\mathrm{C} 42 \mathrm{~B} \quad 59.8(5)$

$\mathrm{C} 43 \mathrm{~B}-\mathrm{C} 43-\mathrm{C} 45 \mathrm{~B} \quad 94.5(5)$

$\mathrm{C} 44 \mathrm{~B}-\mathrm{C} 43-\mathrm{C} 42 \quad 161.0(15)$

$\mathrm{C} 44 \mathrm{~B}-\mathrm{C} 43-\mathrm{H} 43 \mathrm{~A} \quad 56.5$

$\mathrm{C} 44 \mathrm{~B}-\mathrm{C} 43-\mathrm{H} 43 \mathrm{~B} \quad 88.4$

$\mathrm{C} 44 \mathrm{~B}-\mathrm{C} 43-\mathrm{C} 44 \quad 63.7$ (11)

$\mathrm{C} 44 \mathrm{~B}-\mathrm{C} 43-\mathrm{C} 42 \mathrm{~B} \quad 156.7$ (14)

$\mathrm{C} 44 \mathrm{~B}-\mathrm{C} 43-\mathrm{C} 43 \mathrm{~B} \quad 98.2(12)$

$\mathrm{C} 44 \mathrm{~B}-\mathrm{C} 43-\mathrm{C} 45 \mathrm{~B} \quad 7.0(12)$

$\mathrm{C} 45 \mathrm{~B}-\mathrm{C} 43-\mathrm{H} 43 \mathrm{~A} \quad 49.5$

$\mathrm{C} 45 \mathrm{~B}-\mathrm{C} 43-\mathrm{H} 43 \mathrm{~B} \quad 91.3$

$\mathrm{C} 43-\mathrm{C} 44-\mathrm{H} 44 \quad 114.7$

$\mathrm{C} 43-\mathrm{C} 44-\mathrm{C} 43 \mathrm{~B} \quad 43.1$ (4)

$\mathrm{C} 43-\mathrm{C} 44-\mathrm{C} 45 \mathrm{~B} \quad 68.6(4)$

$\mathrm{C} 45-\mathrm{C} 44-\mathrm{C} 43 \quad 130.6(6)$

$\mathrm{C} 45-\mathrm{C} 44-\mathrm{H} 44 \quad 114.7$

$\mathrm{C} 45-\mathrm{C} 44-\mathrm{C} 43 \mathrm{~B} \quad 112.1$ (7)

$\mathrm{C} 45-\mathrm{C} 44-\mathrm{C} 45 \mathrm{~B} \quad 64.7$ (4)

$\mathrm{C} 43 \mathrm{~B}-\mathrm{C} 44-\mathrm{H} 44$ 


\begin{tabular}{|c|c|}
\hline $\mathrm{C} 19-\mathrm{C} 18-\mathrm{C} 17$ & $119.4(2)$ \\
\hline $\mathrm{C} 19-\mathrm{C} 18-\mathrm{H} 18$ & 120.3 \\
\hline $\mathrm{C} 18-\mathrm{C} 19-\mathrm{H} 19$ & 118.9 \\
\hline $\mathrm{C} 18-\mathrm{C} 19-\mathrm{C} 20$ & $122.2(2)$ \\
\hline $\mathrm{C} 20-\mathrm{C} 19-\mathrm{H} 19$ & 118.9 \\
\hline $\mathrm{O} 6-\mathrm{C} 20-\mathrm{C} 19$ & $110.8(2)$ \\
\hline $\mathrm{O} 6-\mathrm{C} 20-\mathrm{C} 21$ & $104.1(2)$ \\
\hline $\mathrm{O} 6-\mathrm{C} 20-\mathrm{C} 22$ & $107.8(2)$ \\
\hline $\mathrm{C} 19-\mathrm{C} 20-\mathrm{C} 21$ & $112.6(2)$ \\
\hline $\mathrm{C} 19-\mathrm{C} 20-\mathrm{C} 22$ & $109.3(2)$ \\
\hline $\mathrm{C} 22-\mathrm{C} 20-\mathrm{C} 21$ & $112.0(2)$ \\
\hline $\mathrm{C} 20-\mathrm{C} 21-\mathrm{H} 21 \mathrm{~A}$ & 109.5 \\
\hline $\mathrm{C} 20-\mathrm{C} 21-\mathrm{H} 21 \mathrm{~B}$ & 109.5 \\
\hline $\mathrm{C} 20-\mathrm{C} 21-\mathrm{H} 21 \mathrm{C}$ & 109.5 \\
\hline $\mathrm{H} 21 \mathrm{~A}-\mathrm{C} 21-\mathrm{H} 21 \mathrm{~B}$ & 109.5 \\
\hline $\mathrm{H} 21 \mathrm{~A}-\mathrm{C} 21-\mathrm{H} 21 \mathrm{C}$ & 109.5 \\
\hline $\mathrm{H} 21 \mathrm{~B}-\mathrm{C} 21-\mathrm{H} 21 \mathrm{C}$ & 109.5 \\
\hline $\mathrm{C} 20-\mathrm{C} 22-\mathrm{H} 22 \mathrm{~A}$ & 109.5 \\
\hline $\mathrm{C} 20-\mathrm{C} 22-\mathrm{H} 22 \mathrm{~B}$ & 109.5 \\
\hline $\mathrm{C} 20-\mathrm{C} 22-\mathrm{H} 22 \mathrm{C}$ & 109.5 \\
\hline $\mathrm{H} 22 \mathrm{~A}-\mathrm{C} 22-\mathrm{H} 22 \mathrm{~B}$ & 109.5 \\
\hline $\mathrm{H} 22 \mathrm{~A}-\mathrm{C} 22-\mathrm{H} 22 \mathrm{C}$ & 109.5 \\
\hline $\mathrm{H} 22 \mathrm{~B}-\mathrm{C} 22-\mathrm{H} 22 \mathrm{C}$ & 109.5 \\
\hline $\mathrm{C} 2 \mathrm{~A}-\mathrm{O} 1 \mathrm{~A}-\mathrm{C} 1 \mathrm{~A}$ & $105.10(19)$ \\
\hline $\mathrm{C} 3 \mathrm{~A}-\mathrm{O} 2 \mathrm{~A}-\mathrm{C} 1 \mathrm{~A}$ & $105.00(18)$ \\
\hline $\mathrm{C} 9 \mathrm{~A}-\mathrm{O} 3 \mathrm{~A}-\mathrm{C} 10 \mathrm{~A}$ & $118.46(17)$ \\
\hline $\mathrm{C} 14 \mathrm{~A}-\mathrm{O} 5 \mathrm{~A}-\mathrm{C} 15 \mathrm{~A}$ & $116.10(19)$ \\
\hline $\mathrm{C} 16 \mathrm{~A}-\mathrm{O} 6 \mathrm{~A}-\mathrm{C} 20 \mathrm{~A}$ & $118.02(18)$ \\
\hline $\mathrm{O} 1 \mathrm{~A}-\mathrm{C} 1 \mathrm{~A}-\mathrm{H} 1 \mathrm{AA}$ & 110.1 \\
\hline $\mathrm{O} 1 \mathrm{~A}-\mathrm{C} 1 \mathrm{~A}-\mathrm{H} 1 \mathrm{AB}$ & 110.1 \\
\hline $\mathrm{O} 2 \mathrm{~A}-\mathrm{C} 1 \mathrm{~A}-\mathrm{O} 1 \mathrm{~A}$ & $107.79(18)$ \\
\hline $\mathrm{O} 2 \mathrm{~A}-\mathrm{C} 1 \mathrm{~A}-\mathrm{H} 1 \mathrm{AA}$ & 110.1 \\
\hline $\mathrm{O} 2 \mathrm{~A}-\mathrm{C} 1 \mathrm{~A}-\mathrm{H} 1 \mathrm{AB}$ & 110.1 \\
\hline $\mathrm{H} 1 \mathrm{AA}-\mathrm{C} 1 \mathrm{~A}-\mathrm{H} 1 \mathrm{AB}$ & 108.5 \\
\hline $\mathrm{C} 3 \mathrm{~A}-\mathrm{C} 2 \mathrm{~A}-\mathrm{O} 1 \mathrm{~A}$ & $109.5(2)$ \\
\hline $\mathrm{C} 7 \mathrm{~A}-\mathrm{C} 2 \mathrm{~A}-\mathrm{O} 1 \mathrm{~A}$ & $127.8(2)$ \\
\hline $\mathrm{C} 7 \mathrm{~A}-\mathrm{C} 2 \mathrm{~A}-\mathrm{C} 3 \mathrm{~A}$ & $122.7(2)$ \\
\hline $\mathrm{C} 2 \mathrm{~A}-\mathrm{C} 3 \mathrm{~A}-\mathrm{O} 2 \mathrm{~A}$ & $110.4(2)$ \\
\hline $\mathrm{C} 4 \mathrm{~A}-\mathrm{C} 3 \mathrm{~A}-\mathrm{O} 2 \mathrm{~A}$ & $127.7(2)$ \\
\hline $\mathrm{C} 4 \mathrm{~A}-\mathrm{C} 3 \mathrm{~A}-\mathrm{C} 2 \mathrm{~A}$ & $122.0(2)$ \\
\hline $\mathrm{C} 3 \mathrm{~A}-\mathrm{C} 4 \mathrm{~A}-\mathrm{H} 4 \mathrm{~A}$ & 121.7 \\
\hline $\mathrm{C} 3 \mathrm{~A}-\mathrm{C} 4 \mathrm{~A}-\mathrm{C} 5 \mathrm{~A}$ & $116.6(2)$ \\
\hline $\mathrm{C} 5 \mathrm{~A}-\mathrm{C} 4 \mathrm{~A}-\mathrm{H} 4 \mathrm{~A}$ & 121.7 \\
\hline $\mathrm{C} 4 \mathrm{~A}-\mathrm{C} 5 \mathrm{~A}-\mathrm{H} 5 \mathrm{~A}$ & 118.9 \\
\hline $\mathrm{C} 6 \mathrm{~A}-\mathrm{C} 5 \mathrm{~A}-\mathrm{C} 4 \mathrm{~A}$ & $122.1(2)$ \\
\hline $\mathrm{C} 6 \mathrm{~A}-\mathrm{C} 5 \mathrm{~A}-\mathrm{H} 5 \mathrm{~A}$ & 118.9 \\
\hline $\mathrm{C} 5 \mathrm{~A}-\mathrm{C} 6 \mathrm{~A}-\mathrm{C} 7 \mathrm{~A}$ & $119.8(2)$ \\
\hline $\mathrm{C} 5 \mathrm{~A}-\mathrm{C} 6 \mathrm{~A}-\mathrm{C} 8 \mathrm{~A}$ & $119.9(2)$ \\
\hline
\end{tabular}

$\begin{array}{ll}\mathrm{C} 43 \mathrm{~B}-\mathrm{C} 44-\mathrm{C} 45 \mathrm{~B} & 80.2(4) \\ \mathrm{C} 44 \mathrm{~B}-\mathrm{C} 44-\mathrm{C} 43 & 27.6(4) \\ \mathrm{C} 44 \mathrm{~B}-\mathrm{C} 44-\mathrm{H} 44 & 138.7 \\ \mathrm{C} 44 \mathrm{~B}-\mathrm{C} 44-\mathrm{C} 45 & 105.0(6) \\ \mathrm{C} 44 \mathrm{~B}-\mathrm{C} 44-\mathrm{C} 43 \mathrm{~B} & 55.2(4) \\ \mathrm{C} 44 \mathrm{~B}-\mathrm{C} 44-\mathrm{C} 45 \mathrm{~B} & 41.2(3) \\ \mathrm{C} 45 \mathrm{~B}-\mathrm{C} 44-\mathrm{H} 44 & 161.6 \\ \mathrm{C} 44-\mathrm{C} 45-\mathrm{C} 46 & 122.5(6) \\ \mathrm{C} 44-\mathrm{C} 45-\mathrm{C} 47 & 123.4(7) \\ \mathrm{C} 44-\mathrm{C} 45-\mathrm{C} 45 \mathrm{~B} & 75.2(4) \\ \mathrm{C} 46-\mathrm{C} 45-\mathrm{C} 47 & 114.1(6) \\ \mathrm{C} 46-\mathrm{C} 45-\mathrm{C} 45 \mathrm{~B} & 52.2(5) \\ \mathrm{C} 47-\mathrm{C} 45-\mathrm{C} 45 \mathrm{~B} & 153.8(6) \\ \mathrm{C} 45-\mathrm{C} 46-\mathrm{H} 46 \mathrm{~A} & 109.5\end{array}$

$\mathrm{C} 45-\mathrm{C} 46-\mathrm{H} 46 \mathrm{~A} \quad 109.5$

$\mathrm{C} 45-\mathrm{C} 46-\mathrm{H} 46 \mathrm{~B} \quad 109.5$

$\mathrm{C} 45-\mathrm{C} 46-\mathrm{H} 46 \mathrm{C}$

$\mathrm{C} 45-\mathrm{C} 46-\mathrm{C} 45 \mathrm{~B} \quad 77.6(5)$

$\mathrm{H} 46 \mathrm{~A}-\mathrm{C} 46-\mathrm{H} 46 \mathrm{~B} \quad 109.5$

$\mathrm{H} 46 \mathrm{~A}-\mathrm{C} 46-\mathrm{H} 46 \mathrm{C} \quad 109.5$

$\mathrm{H} 46 \mathrm{~B}-\mathrm{C} 46-\mathrm{H} 46 \mathrm{C} \quad 109.5$

$\mathrm{C} 45 \mathrm{~B}-\mathrm{C} 46-\mathrm{H} 46 \mathrm{~A} \quad 92.8$

$\mathrm{C} 45 \mathrm{~B}-\mathrm{C} 46-\mathrm{H} 46 \mathrm{~B} \quad 151.7$

$\mathrm{C} 45 \mathrm{~B}-\mathrm{C} 46-\mathrm{H} 46 \mathrm{C} \quad 44.4$

$\mathrm{C} 47 \mathrm{~B}-\mathrm{C} 46-\mathrm{C} 45 \quad 139.3(7)$

$\mathrm{C} 47 \mathrm{~B}-\mathrm{C} 46-\mathrm{H} 46 \mathrm{~A} \quad 55.4$

$\mathrm{C} 47 \mathrm{~B}-\mathrm{C} 46-\mathrm{H} 46 \mathrm{~B} \quad 111.3$

$\mathrm{C} 47 \mathrm{~B}-\mathrm{C} 46-\mathrm{H} 46 \mathrm{C} \quad 56.5$

$\mathrm{C} 47 \mathrm{~B}-\mathrm{C} 46-\mathrm{C} 45 \mathrm{~B} \quad 66.9(6)$

$\mathrm{C} 45-\mathrm{C} 47-\mathrm{H} 47 \mathrm{~A} \quad 109.5$

$\mathrm{C} 45-\mathrm{C} 47-\mathrm{H} 47 \mathrm{~B} \quad 109.5$

$\mathrm{C} 45-\mathrm{C} 47-\mathrm{H} 47 \mathrm{C} \quad 109.5$

$\mathrm{H} 47 \mathrm{~A}-\mathrm{C} 47-\mathrm{H} 47 \mathrm{~B} \quad 109.5$

$\mathrm{H} 47 \mathrm{~A}-\mathrm{C} 47-\mathrm{H} 47 \mathrm{C} \quad 109.5$

$\mathrm{H} 47 \mathrm{~B}-\mathrm{C} 47-\mathrm{H} 47 \mathrm{C} \quad 109.5$

$\mathrm{C} 48-\mathrm{C} 47-\mathrm{C} 45$

$\mathrm{C} 48-\mathrm{C} 47-\mathrm{H} 47 \mathrm{~A} \quad 59.5$

$\mathrm{C} 48-\mathrm{C} 47-\mathrm{H} 47 \mathrm{~B} \quad 69.5$

$\mathrm{C} 48-\mathrm{C} 47-\mathrm{H} 47 \mathrm{C} \quad 83.6$

$\mathrm{C} 40-\mathrm{C} 42 \mathrm{~A}-\mathrm{H} 42 \mathrm{C} \quad 111.0$

$\mathrm{C} 40-\mathrm{C} 42 \mathrm{~A}-\mathrm{H} 42 \mathrm{D} \quad 111.0$

$\mathrm{C} 40-\mathrm{C} 42 \mathrm{~A}-\mathrm{C} 43 \mathrm{~A} \quad 103.6$ (5)

$\mathrm{H} 42 \mathrm{C}-\mathrm{C} 42 \mathrm{~A}-\mathrm{H} 42 \mathrm{D} \quad 109.0$

$\mathrm{C} 43 \mathrm{~A}-\mathrm{C} 42 \mathrm{~A}-\mathrm{H} 42 \mathrm{C} \quad 111.0$

$\mathrm{C} 43 \mathrm{~A}-\mathrm{C} 42 \mathrm{~A}-\mathrm{H} 42 \mathrm{D} \quad 111.0$

$\mathrm{C} 42 \mathrm{~A}-\mathrm{C} 43 \mathrm{~A}-\mathrm{H} 43 \mathrm{C} \quad 107.7$

$\mathrm{C} 42 \mathrm{~A}-\mathrm{C} 43 \mathrm{~A}-\mathrm{H} 43 \mathrm{D} \quad 107.7$

$\mathrm{H} 43 \mathrm{C}-\mathrm{C} 43 \mathrm{~A}-\mathrm{H} 43 \mathrm{D} \quad 107.1$

$\mathrm{C} 44 \mathrm{~A}-\mathrm{C} 43 \mathrm{~A}-\mathrm{C} 42 \mathrm{~A} \quad 118.3$ (7) 


$\begin{array}{ll}\text { C7A-C6A-C8A } & 120.3(2) \\ \text { C2A-C7A-C6A } & 116.7(2) \\ \text { C2A-C7A-H7AA } & 121.6 \\ \text { C6A-C7A-H7AA } & 121.6 \\ \text { C9A-C8A-C6A } & 120.7(2) \\ \text { C9A-C8A-C12A } & 118.3(2) \\ \text { C12A-C8A-C6A } & 121.0(2) \\ \text { O3A-C9A-H9AA } & 117.3 \\ \text { C8A-C9A-O3A } & 125.4(2) \\ \text { C8A-C9A-H9AA } & 117.3 \\ \text { O3A-C10A-C11A } & 121.4(2) \\ \text { O3A-C10A-C17A } & 116.95(19) \\ \text { C11A-C10A-C17A } & 121.7(2) \\ \text { C10A-C11A-C12A } & 120.1(2) \\ \text { C10A-C11A-C13A } & 119.0(2) \\ \text { C13A-C11A-C12A } & 120.9(2) \\ \text { O4A-C12A-C8A } & 122.1(2) \\ \text { O4A-C12A-C11A } & 121.5(2) \\ \text { C11A-C12A-C8A } & 116.31(19) \\ \text { C11A-C13A-H13A } & 119.6 \\ \text { C14A-C13A-C11A } & 120.8(2) \\ \text { C14A-C13A-H13A } & 119.6 \\ \text { O5A-C14A-C13A } & 125.7(2) \\ \text { O5A-C14A-C16A } & 115.2(2) \\ \text { C13A-C14A-C16A } & 119.1(2) \\ \text { O5A-C15A-H15D } & 109.5 \\ \text { O5A-C15A-H15E } & 109.5 \\ \text { O5A-C15A-H15F } & 109.5 \\ \text { H15D-C15A-H15E } & 109.5 \\ \text { H15D-C15A-H15F } & 109.5 \\ \text { H15E-C15A-H15F } & 109.5 \\ \text { O6A-C16A-C14A } & 116.2(2) \\ \text { O6A-C16A-C17A } & 122.1(2) \\ \text { C17A-C16A-C14A } & 121.6(2) \\ \text { C10A-C17A-C18A } & 124.5(2) \\ \text { C16A-C17A-C10A } & 117.8(2) \\ \text { C16A-C17A-C18A } & 117.6(2) \\ \text { C17A-C18A-H18A } & 120.4 \\ \text { C19A-C18A-C17A } & 119.2(2) \\ \text { C19A-C18A-H18A } & 120.4 \\ \text { C18A-C19A-H19A } & 119.2 \\ \text { C18A-C19A-C20A } & 121.6(2) \\ \text { C20A-C19A-H19A } & 119.2 \\ \text { O6A-C20A-C19A } & 109.59(19) \\ \text { O6A-C20A-C21A } & 103.73(19) \\ \text { O6A-C20A-C22A } & 107.68(19) \\ \text { C19A-C20A-C21A } & 113.1(2) \\ \text { C19A-C20A-C22A } & 110.1(2) \\ \text { A } & \end{array}$

\begin{tabular}{|c|c|}
\hline $\mathrm{C} 44 \mathrm{~A}-\mathrm{C} 43 \mathrm{~A}-\mathrm{H} 43 \mathrm{C}$ & 107.7 \\
\hline $\mathrm{C} 44 \mathrm{~A}-\mathrm{C} 43 \mathrm{~A}-\mathrm{H} 43 \mathrm{D}$ & 107.7 \\
\hline $\mathrm{C} 43 \mathrm{~A}-\mathrm{C} 44 \mathrm{~A}-\mathrm{H} 44 \mathrm{~A}$ & 112.4 \\
\hline $\mathrm{C} 45 \mathrm{~A}-\mathrm{C} 44 \mathrm{~A}-\mathrm{C} 43 \mathrm{~A}$ & $135.1(9)$ \\
\hline $\mathrm{C} 45 \mathrm{~A}-\mathrm{C} 44 \mathrm{~A}-\mathrm{H} 44 \mathrm{~A}$ & 112.4 \\
\hline $\mathrm{C} 44 \mathrm{~A}-\mathrm{C} 45 \mathrm{~A}-\mathrm{C} 46 \mathrm{~A}$ & $123.9(9)$ \\
\hline $\mathrm{C} 44 \mathrm{~A}-\mathrm{C} 45 \mathrm{~A}-\mathrm{C} 47 \mathrm{~A}$ & $122.5(10)$ \\
\hline $\mathrm{C} 46 \mathrm{~A}-\mathrm{C} 45 \mathrm{~A}-\mathrm{C} 47 \mathrm{~A}$ & $113.1(9)$ \\
\hline $\mathrm{C} 45 \mathrm{~A}-\mathrm{C} 46 \mathrm{~A}-\mathrm{H} 46 \mathrm{D}$ & 109.5 \\
\hline $\mathrm{C} 45 \mathrm{~A}-\mathrm{C} 46 \mathrm{~A}-\mathrm{H} 46 \mathrm{E}$ & 109.5 \\
\hline $\mathrm{C} 45 \mathrm{~A}-\mathrm{C} 46 \mathrm{~A}-\mathrm{H} 46 \mathrm{~F}$ & 109.5 \\
\hline $\mathrm{H} 46 \mathrm{D}-\mathrm{C} 46 \mathrm{~A}-\mathrm{H} 46 \mathrm{E}$ & 109.5 \\
\hline $\mathrm{H} 46 \mathrm{D}-\mathrm{C} 46 \mathrm{~A}-\mathrm{H} 46 \mathrm{~F}$ & 109.5 \\
\hline $\mathrm{H} 46 \mathrm{E}-\mathrm{C} 46 \mathrm{~A}-\mathrm{H} 46 \mathrm{~F}$ & 109.5 \\
\hline $\mathrm{C} 45 \mathrm{~A}-\mathrm{C} 47 \mathrm{~A}-\mathrm{H} 47 \mathrm{D}$ & 109.5 \\
\hline $\mathrm{C} 45 \mathrm{~A}-\mathrm{C} 47 \mathrm{~A}-\mathrm{H} 47 \mathrm{E}$ & 109.5 \\
\hline $\mathrm{C} 45 \mathrm{~A}-\mathrm{C} 47 \mathrm{~A}-\mathrm{H} 47 \mathrm{~F}$ & 109.5 \\
\hline $\mathrm{H} 47 \mathrm{D}-\mathrm{C} 47 \mathrm{~A}-\mathrm{H} 47 \mathrm{E}$ & 109.5 \\
\hline $\mathrm{H} 47 \mathrm{D}-\mathrm{C} 47 \mathrm{~A}-\mathrm{H} 47 \mathrm{~F}$ & 109.5 \\
\hline $\mathrm{H} 47 \mathrm{E}-\mathrm{C} 47 \mathrm{~A}-\mathrm{H} 47 \mathrm{~F}$ & 109.5 \\
\hline $\mathrm{C} 40-\mathrm{C} 42 \mathrm{~B}-\mathrm{C} 43$ & $104.5(5)$ \\
\hline $\mathrm{C} 40-\mathrm{C} 42 \mathrm{~B}-\mathrm{H} 42 \mathrm{E}$ & 107.5 \\
\hline $\mathrm{C} 40-\mathrm{C} 42 \mathrm{~B}-\mathrm{H} 42 \mathrm{~F}$ & 107.5 \\
\hline $\mathrm{C} 40-\mathrm{C} 42 \mathrm{~B}-\mathrm{C} 43 \mathrm{~B}$ & $119.5(6)$ \\
\hline $\mathrm{C} 43-\mathrm{C} 42 \mathrm{~B}-\mathrm{H} 42 \mathrm{E}$ & 144.7 \\
\hline $\mathrm{C} 43-\mathrm{C} 42 \mathrm{~B}-\mathrm{H} 42 \mathrm{~F}$ & 76.7 \\
\hline $\mathrm{H} 42 \mathrm{E}-\mathrm{C} 42 \mathrm{~B}-\mathrm{H} 42 \mathrm{~F}$ & 107.0 \\
\hline $\mathrm{C} 43 \mathrm{~B}-\mathrm{C} 42 \mathrm{~B}-\mathrm{C} 43$ & $41.6(4)$ \\
\hline $\mathrm{C} 43 \mathrm{~B}-\mathrm{C} 42 \mathrm{~B}-\mathrm{H} 42 \mathrm{E}$ & 107.5 \\
\hline $\mathrm{C} 43 \mathrm{~B}-\mathrm{C} 42 \mathrm{~B}-\mathrm{H} 42 \mathrm{~F}$ & 107.5 \\
\hline $\mathrm{C} 42-\mathrm{C} 43 \mathrm{~B}-\mathrm{C} 44$ & $106.0(7)$ \\
\hline $\mathrm{C} 42-\mathrm{C} 43 \mathrm{~B}-\mathrm{C} 42 \mathrm{~B}$ & $9.4(8)$ \\
\hline $\mathrm{C} 42-\mathrm{C} 43 \mathrm{~B}-\mathrm{H} 43 \mathrm{E}$ & 115.0 \\
\hline $\mathrm{C} 42-\mathrm{C} 43 \mathrm{~B}-\mathrm{H} 43 \mathrm{~F}$ & 115.1 \\
\hline $\mathrm{C} 43-\mathrm{C} 43 \mathrm{~B}-\mathrm{C} 42$ & $69.3(6)$ \\
\hline $\mathrm{C} 43-\mathrm{C} 43 \mathrm{~B}-\mathrm{C} 44$ & $56.3(5)$ \\
\hline $\mathrm{C} 43-\mathrm{C} 43 \mathrm{~B}-\mathrm{C} 42 \mathrm{~B}$ & $78.6(7)$ \\
\hline $\mathrm{C} 43-\mathrm{C} 43 \mathrm{~B}-\mathrm{H} 43 \mathrm{E}$ & 118.0 \\
\hline $\mathrm{C} 43-\mathrm{C} 43 \mathrm{~B}-\mathrm{H} 43 \mathrm{~F}$ & 125.3 \\
\hline $\mathrm{C} 43-\mathrm{C} 43 \mathrm{~B}-\mathrm{C} 44 \mathrm{~B}$ & $27.3(4)$ \\
\hline $\mathrm{C} 44-\mathrm{C} 43 \mathrm{~B}-\mathrm{H} 43 \mathrm{E}$ & 64.3 \\
\hline $\mathrm{C} 44-\mathrm{C} 43 \mathrm{~B}-\mathrm{H} 43 \mathrm{~F}$ & 136.1 \\
\hline $\mathrm{C} 42 \mathrm{~B}-\mathrm{C} 43 \mathrm{~B}-\mathrm{C} 44$ & $112.2(7)$ \\
\hline $\mathrm{C} 42 \mathrm{~B}-\mathrm{C} 43 \mathrm{~B}-\mathrm{H} 43 \mathrm{E}$ & 110.6 \\
\hline $\mathrm{C} 42 \mathrm{~B}-\mathrm{C} 43 \mathrm{~B}-\mathrm{H} 43 \mathrm{~F}$ & 110.6 \\
\hline $\mathrm{H} 43 \mathrm{E}-\mathrm{C} 43 \mathrm{~B}-\mathrm{H} 43 \mathrm{~F}$ & 108.7 \\
\hline $\mathrm{C} 44 \mathrm{~B}-\mathrm{C} 43 \mathrm{~B}-\mathrm{C} 42$ & $96.3(6)$ \\
\hline $\mathrm{C} 44 \mathrm{~B}-\mathrm{C} 43 \mathrm{~B}-\mathrm{C} 44$ & $47.6(6)$ \\
\hline
\end{tabular}




\begin{tabular}{|c|c|}
\hline $\mathrm{C} 21 \mathrm{~A}-\mathrm{C} 20 \mathrm{~A}-\mathrm{C} 22 \mathrm{~A}$ & $112.2(2)$ \\
\hline $\mathrm{C} 20 \mathrm{~A}-\mathrm{C} 21 \mathrm{~A}-\mathrm{H} 21 \mathrm{D}$ & 109.5 \\
\hline $\mathrm{C} 20 \mathrm{~A}-\mathrm{C} 21 \mathrm{~A}-\mathrm{H} 21 \mathrm{E}$ & 109.5 \\
\hline $\mathrm{C} 20 \mathrm{~A}-\mathrm{C} 21 \mathrm{~A}-\mathrm{H} 21 \mathrm{~F}$ & 109.5 \\
\hline $\mathrm{H} 21 \mathrm{D}-\mathrm{C} 21 \mathrm{~A}-\mathrm{H} 21 \mathrm{E}$ & 109.5 \\
\hline $\mathrm{H} 21 \mathrm{D}-\mathrm{C} 21 \mathrm{~A}-\mathrm{H} 21 \mathrm{~F}$ & 109.5 \\
\hline $\mathrm{H} 21 \mathrm{E}-\mathrm{C} 21 \mathrm{~A}-\mathrm{H} 21 \mathrm{~F}$ & 109.5 \\
\hline $\mathrm{C} 20 \mathrm{~A}-\mathrm{C} 22 \mathrm{~A}-\mathrm{H} 22 \mathrm{D}$ & 109.5 \\
\hline $\mathrm{C} 20 \mathrm{~A}-\mathrm{C} 22 \mathrm{~A}-\mathrm{H} 22 \mathrm{E}$ & 109.5 \\
\hline $\mathrm{C} 20 \mathrm{~A}-\mathrm{C} 22 \mathrm{~A}-\mathrm{H} 22 \mathrm{~F}$ & 109.5 \\
\hline $\mathrm{H} 22 \mathrm{D}-\mathrm{C} 22 \mathrm{~A}-\mathrm{H} 22 \mathrm{E}$ & 109.5 \\
\hline $\mathrm{H} 22 \mathrm{D}-\mathrm{C} 22 \mathrm{~A}-\mathrm{H} 22 \mathrm{~F}$ & 109.5 \\
\hline $\mathrm{H} 22 \mathrm{E}-\mathrm{C} 22 \mathrm{~A}-\mathrm{H} 22 \mathrm{~F}$ & 109.5 \\
\hline $\mathrm{C} 23-\mathrm{O} 7-\mathrm{H} 7$ & 109.5 \\
\hline $\mathrm{C} 33-\mathrm{O} 9-\mathrm{H} 9$ & 109.5 \\
\hline $\mathrm{O} 7-\mathrm{C} 23-\mathrm{C} 24$ & $117.8(2)$ \\
\hline $\mathrm{O} 7-\mathrm{C} 23-\mathrm{C} 28$ & $122.7(2)$ \\
\hline $\mathrm{C} 24-\mathrm{C} 23-\mathrm{C} 28$ & $119.5(2)$ \\
\hline $\mathrm{C} 23-\mathrm{C} 24-\mathrm{H} 24$ & 120.2 \\
\hline $\mathrm{C} 25-\mathrm{C} 24-\mathrm{C} 23$ & $119.6(2)$ \\
\hline $\mathrm{C} 25-\mathrm{C} 24-\mathrm{H} 24$ & 120.2 \\
\hline $\mathrm{C} 24-\mathrm{C} 25-\mathrm{H} 25$ & 118.9 \\
\hline $\mathrm{C} 24-\mathrm{C} 25-\mathrm{C} 26$ & $122.1(2)$ \\
\hline $\mathrm{C} 26-\mathrm{C} 25-\mathrm{H} 25$ & 118.9 \\
\hline $\mathrm{C} 25-\mathrm{C} 26-\mathrm{C} 27$ & $117.4(2)$ \\
\hline $\mathrm{C} 25-\mathrm{C} 26-\mathrm{C} 29$ & $120.1(2)$ \\
\hline $\mathrm{C} 27-\mathrm{C} 26-\mathrm{C} 29$ & $122.5(2)$ \\
\hline $\mathrm{C} 26-\mathrm{C} 27-\mathrm{H} 27$ & 119.5 \\
\hline $\mathrm{C} 28-\mathrm{C} 27-\mathrm{C} 26$ & $121.0(2)$ \\
\hline $\mathrm{C} 28-\mathrm{C} 27-\mathrm{H} 27$ & 119.5 \\
\hline $\mathrm{C} 23-\mathrm{C} 28-\mathrm{H} 28$ & 119.8 \\
\hline $\mathrm{C} 27-\mathrm{C} 28-\mathrm{C} 23$ & $120.4(2)$ \\
\hline $\mathrm{C} 27-\mathrm{C} 28-\mathrm{H} 28$ & 119.8 \\
\hline $\mathrm{C} 26-\mathrm{C} 29-\mathrm{H} 29$ & 116.5 \\
\hline $\mathrm{C} 30-\mathrm{C} 29-\mathrm{C} 26$ & $127.0(2)$ \\
\hline $\mathrm{C} 30-\mathrm{C} 29-\mathrm{H} 29$ & 116.5 \\
\hline $\mathrm{C} 29-\mathrm{C} 30-\mathrm{H} 30$ & 118.9 \\
\hline $\mathrm{C} 29-\mathrm{C} 30-\mathrm{C} 31$ & $122.1(2)$ \\
\hline $\mathrm{C} 31-\mathrm{C} 30-\mathrm{H} 30$ & 118.9 \\
\hline $\mathrm{O} 8-\mathrm{C} 31-\mathrm{C} 30$ & $119.2(2)$ \\
\hline $\mathrm{O} 8-\mathrm{C} 31-\mathrm{C} 32$ & $120.2(2)$ \\
\hline $\mathrm{C} 32-\mathrm{C} 31-\mathrm{C} 30$ & $120.6(2)$ \\
\hline $\mathrm{C} 33-\mathrm{C} 32-\mathrm{C} 31$ & $120.2(2)$ \\
\hline $\mathrm{C} 37-\mathrm{C} 32-\mathrm{C} 31$ & $123.4(2)$ \\
\hline C37-C $32-\mathrm{C} 33$ & $116.5(2)$ \\
\hline $\mathrm{O} 9-\mathrm{C} 33-\mathrm{C} 32$ & $121.3(2)$ \\
\hline $\mathrm{O} 9-\mathrm{C} 33-\mathrm{C} 34$ & $116.5(2)$ \\
\hline $\mathrm{C} 34-\mathrm{C} 33-\mathrm{C} 32$ & $122.2(2)$ \\
\hline
\end{tabular}

\begin{tabular}{|c|c|}
\hline $\mathrm{C} 44 \mathrm{~B}-\mathrm{C} 43 \mathrm{~B}-\mathrm{C} 42 \mathrm{~B}$ & $105.7(7)$ \\
\hline $\mathrm{C} 44 \mathrm{~B}-\mathrm{C} 43 \mathrm{~B}-\mathrm{H} 43 \mathrm{E}$ & 110.6 \\
\hline $\mathrm{C} 44 \mathrm{~B}-\mathrm{C} 43 \mathrm{~B}-\mathrm{H} 43 \mathrm{~F}$ & 110.6 \\
\hline $\mathrm{C} 43-\mathrm{C} 44 \mathrm{~B}-\mathrm{C} 44$ & $88.7(12)$ \\
\hline $\mathrm{C} 43-\mathrm{C} 44 \mathrm{~B}-\mathrm{C} 43 \mathrm{~B}$ & $54.5(10)$ \\
\hline $\mathrm{C} 43-\mathrm{C} 44 \mathrm{~B}-\mathrm{H} 44 \mathrm{~B}$ & 66.4 \\
\hline $\mathrm{C} 43-\mathrm{C} 44 \mathrm{~B}-\mathrm{C} 45 \mathrm{~B}$ & $169.5(18)$ \\
\hline $\mathrm{C} 44-\mathrm{C} 44 \mathrm{~B}-\mathrm{C} 43 \mathrm{~B}$ & $77.2(7)$ \\
\hline $\mathrm{C} 44-\mathrm{C} 44 \mathrm{~B}-\mathrm{H} 44 \mathrm{~B}$ & 94.0 \\
\hline $\mathrm{C} 44-\mathrm{C} 44 \mathrm{~B}-\mathrm{C} 45 \mathrm{~B}$ & $98.7(8)$ \\
\hline $\mathrm{C} 43 \mathrm{~B}-\mathrm{C} 44 \mathrm{~B}-\mathrm{H} 44 \mathrm{~B}$ & 120.2 \\
\hline $\mathrm{C} 45 \mathrm{~B}-\mathrm{C} 44 \mathrm{~B}-\mathrm{C} 43 \mathrm{~B}$ & $119.7(7)$ \\
\hline $\mathrm{C} 45 \mathrm{~B}-\mathrm{C} 44 \mathrm{~B}-\mathrm{H} 44 \mathrm{~B}$ & 120.2 \\
\hline $\mathrm{C} 43-\mathrm{C} 45 \mathrm{~B}-\mathrm{C} 44$ & $42.7(2)$ \\
\hline $\mathrm{C} 45-\mathrm{C} 45 \mathrm{~B}-\mathrm{C} 43$ & $81.8(3)$ \\
\hline $\mathrm{C} 45-\mathrm{C} 45 \mathrm{~B}-\mathrm{C} 44$ & $40.1(2)$ \\
\hline $\mathrm{C} 46-\mathrm{C} 45 \mathrm{~B}-\mathrm{C} 43$ & $121.5(6)$ \\
\hline $\mathrm{C} 46-\mathrm{C} 45 \mathrm{~B}-\mathrm{C} 44$ & $87.7(5)$ \\
\hline $\mathrm{C} 46-\mathrm{C} 45 \mathrm{~B}-\mathrm{C} 45$ & $50.1(4)$ \\
\hline $\mathrm{C} 46-\mathrm{C} 45 \mathrm{~B}-\mathrm{C} 47 \mathrm{~B}$ & $46.6(6)$ \\
\hline $\mathrm{C} 44 \mathrm{~B}-\mathrm{C} 45 \mathrm{~B}-\mathrm{C} 43$ & $3.5(6)$ \\
\hline $\mathrm{C} 44 \mathrm{~B}-\mathrm{C} 45 \mathrm{~B}-\mathrm{C} 44$ & $40.2(6)$ \\
\hline $\mathrm{C} 44 \mathrm{~B}-\mathrm{C} 45 \mathrm{~B}-\mathrm{C} 45$ & $79.7(7)$ \\
\hline $\mathrm{C} 44 \mathrm{~B}-\mathrm{C} 45 \mathrm{~B}-\mathrm{C} 46$ & $121.2(8)$ \\
\hline $\mathrm{C} 44 \mathrm{~B}-\mathrm{C} 45 \mathrm{~B}-\mathrm{C} 46 \mathrm{~B}$ & $111.0(7)$ \\
\hline $\mathrm{C} 44 \mathrm{~B}-\mathrm{C} 45 \mathrm{~B}-\mathrm{C} 47 \mathrm{~B}$ & $132.2(8)$ \\
\hline $\mathrm{C} 46 \mathrm{~B}-\mathrm{C} 45 \mathrm{~B}-\mathrm{C} 43$ & $113.4(7)$ \\
\hline $\mathrm{C} 46 \mathrm{~B}-\mathrm{C} 45 \mathrm{~B}-\mathrm{C} 44$ & $108.2(7)$ \\
\hline $\mathrm{C} 46 \mathrm{~B}-\mathrm{C} 45 \mathrm{~B}-\mathrm{C} 45$ & $104.7(7)$ \\
\hline $\mathrm{C} 46 \mathrm{~B}-\mathrm{C} 45 \mathrm{~B}-\mathrm{C} 46$ & $110.0(7)$ \\
\hline $\mathrm{C} 46 \mathrm{~B}-\mathrm{C} 45 \mathrm{~B}-\mathrm{C} 47 \mathrm{~B}$ & $116.2(7)$ \\
\hline $\mathrm{C} 47 \mathrm{~B}-\mathrm{C} 45 \mathrm{~B}-\mathrm{C} 43$ & $129.4(7)$ \\
\hline $\mathrm{C} 47 \mathrm{~B}-\mathrm{C} 45 \mathrm{~B}-\mathrm{C} 44$ & $123.9(8)$ \\
\hline $\mathrm{C} 47 \mathrm{~B}-\mathrm{C} 45 \mathrm{~B}-\mathrm{C} 45$ & $94.7(8)$ \\
\hline $\mathrm{C} 45 \mathrm{~B}-\mathrm{C} 46 \mathrm{~B}-\mathrm{H} 46 \mathrm{G}$ & 109.5 \\
\hline $\mathrm{C} 45 \mathrm{~B}-\mathrm{C} 46 \mathrm{~B}-\mathrm{H} 46 \mathrm{H}$ & 109.5 \\
\hline $\mathrm{C} 45 \mathrm{~B}-\mathrm{C} 46 \mathrm{~B}-\mathrm{H} 46 \mathrm{I}$ & 109.5 \\
\hline $\mathrm{H} 46 \mathrm{G}-\mathrm{C} 46 \mathrm{~B}-\mathrm{H} 46 \mathrm{H}$ & 109.5 \\
\hline $\mathrm{H} 46 \mathrm{G}-\mathrm{C} 46 \mathrm{~B}-\mathrm{H} 46 \mathrm{I}$ & 109.5 \\
\hline $\mathrm{H} 46 \mathrm{H}-\mathrm{C} 46 \mathrm{~B}-\mathrm{H} 46 \mathrm{I}$ & 109.5 \\
\hline $\mathrm{C} 46-\mathrm{C} 47 \mathrm{~B}-\mathrm{C} 45 \mathrm{~B}$ & $66.5(7)$ \\
\hline $\mathrm{C} 46-\mathrm{C} 47 \mathrm{~B}-\mathrm{H} 47 \mathrm{G}$ & 95.1 \\
\hline $\mathrm{C} 46-\mathrm{C} 47 \mathrm{~B}-\mathrm{H} 47 \mathrm{H}$ & 53.6 \\
\hline $\mathrm{C} 46-\mathrm{C} 47 \mathrm{~B}-\mathrm{H} 47 \mathrm{I}$ & 154.6 \\
\hline $\mathrm{C} 45 \mathrm{~B}-\mathrm{C} 47 \mathrm{~B}-\mathrm{H} 47 \mathrm{G}$ & 109.5 \\
\hline $\mathrm{C} 45 \mathrm{~B}-\mathrm{C} 47 \mathrm{~B}-\mathrm{H} 47 \mathrm{H}$ & 109.5 \\
\hline $\mathrm{C} 45 \mathrm{~B}-\mathrm{C} 47 \mathrm{~B}-\mathrm{H} 47 \mathrm{I}$ & 109.5 \\
\hline $\mathrm{H} 47 \mathrm{G}-\mathrm{C} 47 \mathrm{~B}-\mathrm{H} 47 \mathrm{H}$ & 109.5 \\
\hline
\end{tabular}




\begin{tabular}{|c|c|}
\hline $\mathrm{C} 33-\mathrm{C} 34-\mathrm{H} 34$ & 120.4 \\
\hline $\mathrm{C} 35-\mathrm{C} 34-\mathrm{C} 33$ & $119.1(2)$ \\
\hline $\mathrm{C} 35-\mathrm{C} 34-\mathrm{H} 34$ & 120.4 \\
\hline $\mathrm{C} 34-\mathrm{C} 35-\mathrm{C} 36$ & $120.1(2)$ \\
\hline $\mathrm{O} 10-\mathrm{C} 35-\mathrm{C} 34$ & $124.7(2)$ \\
\hline $\mathrm{O} 10-\mathrm{C} 35-\mathrm{C} 36$ & $115.1(2)$ \\
\hline $\mathrm{C} 35-\mathrm{C} 36-\mathrm{H} 36$ & 119.9 \\
\hline $\mathrm{C} 37-\mathrm{C} 36-\mathrm{C} 35$ & $120.1(2)$ \\
\hline C $37-\mathrm{C} 36-\mathrm{H} 36$ & 119.9 \\
\hline $\mathrm{C} 35-\mathrm{O} 10-\mathrm{C} 38$ & $117.74(18)$ \\
\hline C32-C37-H37 & 119.0 \\
\hline $\mathrm{C} 36-\mathrm{C} 37-\mathrm{C} 32$ & $121.9(2)$ \\
\hline C36-C37-H37 & 119.0 \\
\hline $\mathrm{O} 10-\mathrm{C} 38-\mathrm{H} 38 \mathrm{~A}$ & 110.4 \\
\hline $\mathrm{O} 10-\mathrm{C} 38-\mathrm{H} 38 \mathrm{~B}$ & 110.4 \\
\hline $\mathrm{O} 10-\mathrm{C} 38-\mathrm{C} 39$ & $106.7(2)$ \\
\hline $\mathrm{H} 38 \mathrm{~A}-\mathrm{C} 38-\mathrm{H} 38 \mathrm{~B}$ & 108.6 \\
\hline C39-C $38-\mathrm{H} 38 \mathrm{~A}$ & 110.4 \\
\hline $\mathrm{C} 39-\mathrm{C} 38-\mathrm{H} 38 \mathrm{~B}$ & 110.4 \\
\hline $\mathrm{O} 1-\mathrm{C} 2-\mathrm{C} 3-\mathrm{O} 2$ & $-1.6(3)$ \\
\hline $\mathrm{O} 1-\mathrm{C} 2-\mathrm{C} 3-\mathrm{C} 4$ & $175.8(2)$ \\
\hline $\mathrm{O} 1-\mathrm{C} 2-\mathrm{C} 7-\mathrm{C} 6$ & $-177.7(2)$ \\
\hline $\mathrm{O} 2-\mathrm{C} 3-\mathrm{C} 4-\mathrm{C} 5$ & $179.7(2)$ \\
\hline $\mathrm{O} 3-\mathrm{C} 10-\mathrm{C} 11-\mathrm{C} 12$ & $-1.6(4)$ \\
\hline $\mathrm{O} 3-\mathrm{C} 10-\mathrm{C} 11-\mathrm{C} 13$ & $177.2(2)$ \\
\hline $\mathrm{O} 3-\mathrm{C} 10-\mathrm{C} 17-\mathrm{C} 16$ & $-179.1(2)$ \\
\hline $\mathrm{O} 3-\mathrm{C} 10-\mathrm{C} 17-\mathrm{C} 18$ & $-3.4(4)$ \\
\hline $\mathrm{O} 5-\mathrm{C} 14-\mathrm{C} 16-\mathrm{O} 6$ & $0.6(3)$ \\
\hline $\mathrm{O} 5-\mathrm{C} 14-\mathrm{C} 16-\mathrm{C} 17$ & $176.2(2)$ \\
\hline $\mathrm{O} 6-\mathrm{C} 16-\mathrm{C} 17-\mathrm{C} 10$ & $178.2(2)$ \\
\hline $\mathrm{O} 6-\mathrm{C} 16-\mathrm{C} 17-\mathrm{C} 18$ & $2.2(4)$ \\
\hline $\mathrm{C} 1-\mathrm{O} 1-\mathrm{C} 2-\mathrm{C} 3$ & $17.1(3)$ \\
\hline $\mathrm{C} 1-\mathrm{O} 1-\mathrm{C} 2-\mathrm{C} 7$ & $-164.2(3)$ \\
\hline $\mathrm{C} 1-\mathrm{O} 2-\mathrm{C} 3-\mathrm{C} 2$ & $-14.7(3)$ \\
\hline $\mathrm{C} 1-\mathrm{O} 2-\mathrm{C} 3-\mathrm{C} 4$ & 168.0 \\
\hline $\mathrm{C} 2-\mathrm{O} 1-\mathrm{C} 1-\mathrm{O} 2$ & $-26.3(3)$ \\
\hline $\mathrm{C} 2-\mathrm{C} 3-\mathrm{C} 4-\mathrm{C} 5$ & $2.7(4)$ \\
\hline $\mathrm{C} 3-\mathrm{O} 2-\mathrm{C} 1-\mathrm{O} 1$ & $24.8(3)$ \\
\hline $\mathrm{C} 3-\mathrm{C} 2-\mathrm{C} 7-\mathrm{C} 6$ & $0.7(4)$ \\
\hline $\mathrm{C} 3-\mathrm{C} 4-\mathrm{C} 5-\mathrm{C} 6$ & $-0.4(4)$ \\
\hline $\mathrm{C} 4-\mathrm{C} 5-\mathrm{C} 6-\mathrm{C} 7$ & $-1.6(4)$ \\
\hline $\mathrm{C} 4-\mathrm{C} 5-\mathrm{C} 6-\mathrm{C} 8$ & $174.0(2)$ \\
\hline $\mathrm{C} 5-\mathrm{C} 6-\mathrm{C} 7-\mathrm{C} 2$ & $1.5(3)$ \\
\hline $\mathrm{C} 5-\mathrm{C} 6-\mathrm{C} 8-\mathrm{C} 9$ & $-37.0(3)$ \\
\hline $\mathrm{C} 5-\mathrm{C} 6-\mathrm{C} 8-\mathrm{C} 12$ & $145.1(2)$ \\
\hline $\mathrm{C} 6-\mathrm{C} 8-\mathrm{C} 9-\mathrm{O} 3$ & $178.3(2)$ \\
\hline $\mathrm{C} 6-\mathrm{C} 8-\mathrm{C12}-\mathrm{O} 4$ & \\
\hline
\end{tabular}

$\begin{array}{ll}\text { H47G-C47B-H47I } & 109.5 \\ \text { H47H-C47B-H47I } & 109.5 \\ \text { C48-O11-H11 } & 109.5 \\ \text { C47-C48-O11 } & 140.1(19) \\ \text { C47-C48-H48A } & 67.5 \\ \text { C47-C48-H48B } & 44.0 \\ \text { C47-C48-C49 } & 110(2) \\ \text { O11-C48-H48A } & 110.2 \\ \text { O11-C48-H48B } & 110.2 \\ \text { O11-C48-C49 } & 107.4(19) \\ \text { H48A-C48-H48B } & 108.5 \\ \text { C49-C48-H48A } & 110.2 \\ \text { C49-C48-H48B } & 110.2 \\ \text { C48-C49-H49A } & 109.5 \\ \text { C48-C49-H49B } & 109.5 \\ \text { C48-C49-H49C } & 109.5 \\ \text { H49A-C49-H49B } & 109.5 \\ \text { H49A-C49-H49C } & 109.5 \\ \text { H49B-C49-H49C } & 109.5\end{array}$

$\mathrm{C} 40-\mathrm{C} 42-\mathrm{C} 43 \mathrm{~B}-\mathrm{C} 43 \quad 95.1(9)$

$\mathrm{C} 40-\mathrm{C} 42-\mathrm{C} 43 \mathrm{~B}-\mathrm{C} 44 \quad 138.7(8)$

$\mathrm{C} 40-\mathrm{C} 42-\mathrm{C} 43 \mathrm{~B}-\mathrm{C} 42 \mathrm{~B} \quad-89$ (3)

$\mathrm{C} 40-\mathrm{C} 42-\mathrm{C} 43 \mathrm{~B}-\mathrm{C} 44 \mathrm{~B} \quad 91.1(10)$

$\mathrm{C} 40-\mathrm{C} 42 \mathrm{~A}-\mathrm{C} 43 \mathrm{~A}-\mathrm{C} 44 \mathrm{~A} \quad 165.2(9)$

$\mathrm{C} 40-\mathrm{C} 42 \mathrm{~B}-\mathrm{C} 43 \mathrm{~B}-\mathrm{C} 42 \quad 74.8(16)$

$\mathrm{C} 40-\mathrm{C} 42 \mathrm{~B}-\mathrm{C} 43 \mathrm{~B}-\mathrm{C} 43 \quad 78.3(10)$

$\mathrm{C} 40-\mathrm{C} 42 \mathrm{~B}-\mathrm{C} 43 \mathrm{~B}-\mathrm{C} 44 \quad 124.4(9)$

$\mathrm{C} 40-\mathrm{C} 42 \mathrm{~B}-\mathrm{C} 43 \mathrm{~B}-\mathrm{C} 44 \mathrm{~B} \quad 74.4$ (12)

$\mathrm{C} 41-\mathrm{C} 40-\mathrm{C} 42-\mathrm{C} 43 \quad 76.7$ (9)

$\mathrm{C} 41-\mathrm{C} 40-\mathrm{C} 42-\mathrm{C} 43 \mathrm{~B} \quad 24.2(10)$

$\mathrm{C} 41-\mathrm{C} 40-\mathrm{C} 42 \mathrm{~A}-\mathrm{C} 43 \mathrm{~A} \quad 49.9(9)$

$\mathrm{C} 41-\mathrm{C} 40-\mathrm{C} 42 \mathrm{~B}-\mathrm{C} 43 \quad 87.2(7)$

$\mathrm{C} 41-\mathrm{C} 40-\mathrm{C} 42 \mathrm{~B}-\mathrm{C} 43 \mathrm{~B} \quad 45.0(10)$

$\mathrm{C} 42-\mathrm{C} 40-\mathrm{C} 42 \mathrm{~B}-\mathrm{C} 43 \quad-26(3)$

$\mathrm{C} 42-\mathrm{C} 40-\mathrm{C} 42 \mathrm{~B}-\mathrm{C} 43 \mathrm{~B}-68$ (3)

$\mathrm{C} 42-\mathrm{C} 43-\mathrm{C} 44-\mathrm{C} 45 \quad 133.7(10)$

$\mathrm{C} 42-\mathrm{C} 43-\mathrm{C} 44-\mathrm{C} 43 \mathrm{~B} \quad 54.7(8)$

$\mathrm{C} 42-\mathrm{C} 43-\mathrm{C} 44-\mathrm{C} 44 \mathrm{~B} \quad 158.7(16)$

$\mathrm{C} 42-\mathrm{C} 43-\mathrm{C} 44-\mathrm{C} 45 \mathrm{~B} \quad 153.4(8)$

$\mathrm{C} 42-\mathrm{C} 43-\mathrm{C} 42 \mathrm{~B}-\mathrm{C} 40 \quad 55(4)$

$\mathrm{C} 42-\mathrm{C} 43-\mathrm{C} 42 \mathrm{~B}-\mathrm{C} 43 \mathrm{~B} \quad 174$ (4)

$\mathrm{C} 42-\mathrm{C} 43-\mathrm{C} 43 \mathrm{~B}-\mathrm{C} 44 \quad-127.3$ (5)

$\mathrm{C} 42-\mathrm{C} 43-\mathrm{C} 43 \mathrm{~B}-\mathrm{C} 42 \mathrm{~B}-0.6(3)$

$\mathrm{C} 42-\mathrm{C} 43-\mathrm{C} 43 \mathrm{~B}-\mathrm{C} 44 \mathrm{~B} \quad 171.2(14)$

$\mathrm{C} 42-\mathrm{C} 43-\mathrm{C} 44 \mathrm{~B}-\mathrm{C} 44 \quad-100$ (4)

$\mathrm{C} 42-\mathrm{C} 43-\mathrm{C} 44 \mathrm{~B}-\mathrm{C} 43 \mathrm{~B} \quad-25$ (4)

$\mathrm{C} 42-\mathrm{C} 43-\mathrm{C} 44 \mathrm{~B}-\mathrm{C} 45 \mathrm{~B} \quad 34$ (11) 


\begin{tabular}{|c|c|}
\hline $\mathrm{C} 6-\mathrm{C} 8-\mathrm{C} 12-\mathrm{C} 11$ & $-178.7(2)$ \\
\hline $\mathrm{C} 7-\mathrm{C} 2-\mathrm{C} 3-\mathrm{O} 2$ & $179.7(2)$ \\
\hline $\mathrm{C} 7-\mathrm{C} 2-\mathrm{C} 3-\mathrm{C} 4$ & $-2.9(4)$ \\
\hline $\mathrm{C} 7-\mathrm{C} 6-\mathrm{C} 8-\mathrm{C} 9$ & $138.6(2)$ \\
\hline $\mathrm{C} 7-\mathrm{C} 6-\mathrm{C} 8-\mathrm{C} 12$ & $-39.3(3)$ \\
\hline $\mathrm{C} 8-\mathrm{C} 6-\mathrm{C} 7-\mathrm{C} 2$ & $-174.2(2)$ \\
\hline $\mathrm{C} 9-\mathrm{O} 3-\mathrm{C} 10-\mathrm{C} 11$ & $1.7(3)$ \\
\hline $\mathrm{C} 9-\mathrm{O} 3-\mathrm{C} 10-\mathrm{C} 17$ & $-179.2(2)$ \\
\hline $\mathrm{C} 9-\mathrm{C} 8-\mathrm{C} 12-\mathrm{O} 4$ & $-176.4(2)$ \\
\hline $\mathrm{C} 9-\mathrm{C} 8-\mathrm{C} 12-\mathrm{C} 11$ & $3.5(3)$ \\
\hline $\mathrm{C} 10-\mathrm{O} 3-\mathrm{C} 9-\mathrm{C} 8$ & $1.1(4)$ \\
\hline $\mathrm{C} 10-\mathrm{C} 11-\mathrm{C} 12-\mathrm{O} 4$ & $178.9(2)$ \\
\hline $\mathrm{C} 10-\mathrm{C} 11-\mathrm{C} 12-\mathrm{C} 8$ & $-1.0(3)$ \\
\hline $\mathrm{C} 10-\mathrm{C} 11-\mathrm{C} 13-\mathrm{C} 14$ & $0.9(4)$ \\
\hline $\mathrm{C} 10-\mathrm{C} 17-\mathrm{C} 18-\mathrm{C} 19$ & $174.1(3)$ \\
\hline $\mathrm{C} 11-\mathrm{C} 10-\mathrm{C} 17-\mathrm{C} 16$ & $0.0(4)$ \\
\hline $\mathrm{C} 11-\mathrm{C} 10-\mathrm{C} 17-\mathrm{C} 18$ & $175.7(2)$ \\
\hline $\mathrm{C} 11-\mathrm{C} 13-\mathrm{C} 14-\mathrm{O} 5$ & $-178.1(2)$ \\
\hline $\mathrm{C} 11-\mathrm{C} 13-\mathrm{C} 14-\mathrm{C} 16$ & $1.7(4)$ \\
\hline $\mathrm{C} 12-\mathrm{C} 8-\mathrm{C} 9-\mathrm{O} 3$ & $-3.7(4)$ \\
\hline $\mathrm{C} 12-\mathrm{C} 11-\mathrm{C} 13-\mathrm{C} 14$ & $179.7(2)$ \\
\hline $\mathrm{C} 13-\mathrm{C} 11-\mathrm{C} 12-\mathrm{O} 4$ & $0.1(4)$ \\
\hline $\mathrm{C} 13-\mathrm{C} 11-\mathrm{C} 12-\mathrm{C} 8$ & $-179.7(2)$ \\
\hline $\mathrm{C} 13-\mathrm{C} 14-\mathrm{C} 16-\mathrm{O} 6$ & $-179.3(2)$ \\
\hline $\mathrm{C} 13-\mathrm{C} 14-\mathrm{C} 16-\mathrm{C} 17$ & $-3.7(4)$ \\
\hline $\mathrm{C} 14-\mathrm{C} 16-\mathrm{C} 17-\mathrm{C} 10$ & $2.8(4)$ \\
\hline $\mathrm{C} 14-\mathrm{C} 16-\mathrm{C} 17-\mathrm{C} 18$ & $-173.2(2)$ \\
\hline $\mathrm{C} 15-\mathrm{O} 5-\mathrm{C} 14-\mathrm{C} 13$ & $4.1(4)$ \\
\hline $\mathrm{C} 15-\mathrm{O} 5-\mathrm{C} 14-\mathrm{C} 16$ & $-175.8(2)$ \\
\hline $\mathrm{C} 16-\mathrm{O} 6-\mathrm{C} 20-\mathrm{C} 19$ & $-33.5(3)$ \\
\hline $\mathrm{C} 16-\mathrm{O} 6-\mathrm{C} 20-\mathrm{C} 21$ & $-154.8(2)$ \\
\hline $\mathrm{C} 16-\mathrm{O} 6-\mathrm{C} 20-\mathrm{C} 22$ & $86.1(3)$ \\
\hline $\mathrm{C} 16-\mathrm{C} 17-\mathrm{C} 18-\mathrm{C} 19$ & $-10.2(4)$ \\
\hline $\mathrm{C} 17-\mathrm{C} 10-\mathrm{C} 11-\mathrm{C} 12$ & $179.4(2)$ \\
\hline $\mathrm{C} 17-\mathrm{C} 10-\mathrm{C} 11-\mathrm{C} 13$ & $-1.9(4)$ \\
\hline $\mathrm{C} 17-\mathrm{C} 18-\mathrm{C} 19-\mathrm{C} 20$ & $-5.4(4)$ \\
\hline $\mathrm{C} 18-\mathrm{C} 19-\mathrm{C} 20-\mathrm{O} 6$ & $26.2(4)$ \\
\hline $\mathrm{C} 18-\mathrm{C} 19-\mathrm{C} 20-\mathrm{C} 21$ & $142.3(3)$ \\
\hline $\mathrm{C} 18-\mathrm{C} 19-\mathrm{C} 20-\mathrm{C} 22$ & $-92.5(3)$ \\
\hline $\mathrm{C} 20-\mathrm{O} 6-\mathrm{C} 16-\mathrm{C} 14$ & $-163.3(2)$ \\
\hline $\mathrm{C} 20-\mathrm{O} 6-\mathrm{C} 16-\mathrm{C} 17$ & $21.1(4)$ \\
\hline $\mathrm{O} 1 \mathrm{~A}-\mathrm{C} 2 \mathrm{~A}-\mathrm{C} 3 \mathrm{~A}-\mathrm{O} 2 \mathrm{~A}$ & $-0.3(3)$ \\
\hline $\mathrm{O} 1 \mathrm{~A}-\mathrm{C} 2 \mathrm{~A}-\mathrm{C} 3 \mathrm{~A}-\mathrm{C} 4 \mathrm{~A}$ & $-179.7(2)$ \\
\hline $\mathrm{O} 1 \mathrm{~A}-\mathrm{C} 2 \mathrm{~A}-\mathrm{C} 7 \mathrm{~A}-\mathrm{C} 6 \mathrm{~A}$ & $-178.9(2)$ \\
\hline $\mathrm{O} 2 \mathrm{~A}-\mathrm{C} 3 \mathrm{~A}-\mathrm{C} 4 \mathrm{~A}-\mathrm{C} 5 \mathrm{~A}$ & $179.3(2)$ \\
\hline $\mathrm{O} 3 \mathrm{~A}-\mathrm{C} 10 \mathrm{~A}-\mathrm{C} 11 \mathrm{~A}-\mathrm{C} 12 \mathrm{~A}$ & $-1.4(4)$ \\
\hline $\mathrm{O} 3 \mathrm{~A}-\mathrm{C} 10 \mathrm{~A}-\mathrm{C} 11 \mathrm{~A}-\mathrm{C} 13 \mathrm{~A}$ & $178.4(2)$ \\
\hline $\mathrm{O} 3 \mathrm{~A}-\mathrm{C} 10 \mathrm{~A}-\mathrm{C} 17 \mathrm{~A}-\mathrm{C} 16 \mathrm{~A}$ & $178.5(2)$ \\
\hline
\end{tabular}

\begin{tabular}{|c|c|}
\hline $\mathrm{C} 42-\mathrm{C} 43 \mathrm{~B}-\mathrm{C} 44 \mathrm{~B}-\mathrm{C} 43$ & $8.2(13)$ \\
\hline $\mathrm{C} 42-\mathrm{C} 43 \mathrm{~B}-\mathrm{C} 44 \mathrm{~B}-\mathrm{C} 44$ & $105.9(7)$ \\
\hline $\mathrm{C} 42-\mathrm{C} 43 \mathrm{~B}-\mathrm{C} 44 \mathrm{~B}-\mathrm{C} 45 \mathrm{~B}$ & $-161.3(10)$ \\
\hline $\mathrm{C} 43-\mathrm{C} 42-\mathrm{C} 43 \mathrm{~B}-\mathrm{C} 44$ & $43.6(4)$ \\
\hline $\mathrm{C} 43-\mathrm{C} 42-\mathrm{C} 43 \mathrm{~B}-\mathrm{C} 42 \mathrm{~B}$ & $176(2)$ \\
\hline $\mathrm{C} 43-\mathrm{C} 42-\mathrm{C} 43 \mathrm{~B}-\mathrm{C} 44 \mathrm{~B}$ & $-4.0(7)$ \\
\hline $\mathrm{C} 43-\mathrm{C} 44-\mathrm{C} 45-\mathrm{C} 46$ & $-2.9(15)$ \\
\hline $\mathrm{C} 43-\mathrm{C} 44-\mathrm{C} 45-\mathrm{C} 47$ & $179.8(9)$ \\
\hline $\mathrm{C} 43-\mathrm{C} 44-\mathrm{C} 45-\mathrm{C} 45 \mathrm{~B}$ & $20.3(10)$ \\
\hline $\mathrm{C} 43-\mathrm{C} 44-\mathrm{C} 43 \mathrm{~B}-\mathrm{C} 42$ & $-50.7(5)$ \\
\hline $\mathrm{C} 43-\mathrm{C} 44-\mathrm{C} 43 \mathrm{~B}-\mathrm{C} 42 \mathrm{~B}$ & $-58.1(7)$ \\
\hline $\mathrm{C} 43-\mathrm{C} 44-\mathrm{C} 43 \mathrm{~B}-\mathrm{C} 44 \mathrm{~B}$ & $33.2(7)$ \\
\hline $\mathrm{C} 43-\mathrm{C} 44-\mathrm{C} 44 \mathrm{~B}-\mathrm{C} 43 \mathrm{~B}$ & $-53.8(11)$ \\
\hline $\mathrm{C} 43-\mathrm{C} 44-\mathrm{C} 44 \mathrm{~B}-\mathrm{C} 45 \mathrm{~B}$ & $-172.4(15)$ \\
\hline $\mathrm{C} 43-\mathrm{C} 42 \mathrm{~B}-\mathrm{C} 43 \mathrm{~B}-\mathrm{C} 42$ & $-4(2)$ \\
\hline $\mathrm{C} 43-\mathrm{C} 42 \mathrm{~B}-\mathrm{C} 43 \mathrm{~B}-\mathrm{C} 44$ & $46.1(5)$ \\
\hline $\mathrm{C} 43-\mathrm{C} 42 \mathrm{~B}-\mathrm{C} 43 \mathrm{~B}-\mathrm{C} 44 \mathrm{~B}$ & $-3.9(7)$ \\
\hline $\mathrm{C} 43-\mathrm{C} 43 \mathrm{~B}-\mathrm{C} 44 \mathrm{~B}-\mathrm{C} 44$ & $97.6(14)$ \\
\hline $\mathrm{C} 43-\mathrm{C} 43 \mathrm{~B}-\mathrm{C} 44 \mathrm{~B}-\mathrm{C} 45 \mathrm{~B}$ & $-170(2)$ \\
\hline $\mathrm{C} 43-\mathrm{C} 44 \mathrm{~B}-\mathrm{C} 45 \mathrm{~B}-\mathrm{C} 44$ & $-134(8)$ \\
\hline $\mathrm{C} 43-\mathrm{C} 44 \mathrm{~B}-\mathrm{C} 45 \mathrm{~B}-\mathrm{C} 45$ & $-126(8)$ \\
\hline $\mathrm{C} 43-\mathrm{C} 44 \mathrm{~B}-\mathrm{C} 45 \mathrm{~B}-\mathrm{C} 46$ & $-96(8)$ \\
\hline $\mathrm{C} 43-\mathrm{C} 44 \mathrm{~B}-\mathrm{C} 45 \mathrm{~B}-\mathrm{C} 46 \mathrm{~B}$ & $133(8)$ \\
\hline $\mathrm{C} 43-\mathrm{C} 44 \mathrm{~B}-\mathrm{C} 45 \mathrm{~B}-\mathrm{C} 47 \mathrm{~B}$ & $-38(8)$ \\
\hline $\mathrm{C} 43-\mathrm{C} 45 \mathrm{~B}-\mathrm{C} 47 \mathrm{~B}-\mathrm{C} 46$ & $-98.8(10)$ \\
\hline $\mathrm{C} 44-\mathrm{C} 43-\mathrm{C} 42 \mathrm{~B}-\mathrm{C} 40$ & $-179.3(6)$ \\
\hline $\mathrm{C} 44-\mathrm{C} 43-\mathrm{C} 42 \mathrm{~B}-\mathrm{C} 43 \mathrm{~B}$ & $-61.0(8)$ \\
\hline $\mathrm{C} 44-\mathrm{C} 43-\mathrm{C} 43 \mathrm{~B}-\mathrm{C} 42$ & $127.3(5)$ \\
\hline $\mathrm{C} 44-\mathrm{C} 43-\mathrm{C} 43 \mathrm{~B}-\mathrm{C} 42 \mathrm{~B}$ & $126.7(5)$ \\
\hline $\mathrm{C} 44-\mathrm{C} 43-\mathrm{C} 43 \mathrm{~B}-\mathrm{C} 44 \mathrm{~B}$ & $-61.5(12)$ \\
\hline $\mathrm{C} 44-\mathrm{C} 43-\mathrm{C} 44 \mathrm{~B}-\mathrm{C} 43 \mathrm{~B}$ & $75.2(10)$ \\
\hline $\mathrm{C} 44-\mathrm{C} 43-\mathrm{C} 44 \mathrm{~B}-\mathrm{C} 45 \mathrm{~B}$ & $134(8)$ \\
\hline $\mathrm{C} 44-\mathrm{C} 45-\mathrm{C} 46-\mathrm{C} 45 \mathrm{~B}$ & $28.9(9)$ \\
\hline $\mathrm{C} 44-\mathrm{C} 45-\mathrm{C} 46-\mathrm{C} 47 \mathrm{~B}$ & $-0.5(19)$ \\
\hline $\mathrm{C} 44-\mathrm{C} 45-\mathrm{C} 47-\mathrm{C} 48$ & $-127(6)$ \\
\hline $\mathrm{C} 44-\mathrm{C} 45-\mathrm{C} 45 \mathrm{~B}-\mathrm{C} 43$ & $-11.2(6)$ \\
\hline $\mathrm{C} 44-\mathrm{C} 45-\mathrm{C} 45 \mathrm{~B}-\mathrm{C} 46$ & $-155.1(8)$ \\
\hline $\mathrm{C} 44-\mathrm{C} 45-\mathrm{C} 45 \mathrm{~B}-\mathrm{C} 44 \mathrm{~B}$ & $-8.3(7)$ \\
\hline $\mathrm{C} 44-\mathrm{C} 45-\mathrm{C} 45 \mathrm{~B}-\mathrm{C} 46 \mathrm{~B}$ & $101.0(8)$ \\
\hline $\mathrm{C} 44-\mathrm{C} 45-\mathrm{C} 45 \mathrm{~B}-\mathrm{C} 47 \mathrm{~B}$ & $-140.4(7)$ \\
\hline $\mathrm{C} 44-\mathrm{C} 43 \mathrm{~B}-\mathrm{C} 44 \mathrm{~B}-\mathrm{C} 43$ & $-97.6(14)$ \\
\hline $\mathrm{C} 44-\mathrm{C} 43 \mathrm{~B}-\mathrm{C} 44 \mathrm{~B}-\mathrm{C} 45 \mathrm{~B}$ & $92.8(11)$ \\
\hline $\mathrm{C} 44-\mathrm{C} 44 \mathrm{~B}-\mathrm{C} 45 \mathrm{~B}-\mathrm{C} 43$ & $134(8)$ \\
\hline $\mathrm{C} 44-\mathrm{C} 44 \mathrm{~B}-\mathrm{C} 45 \mathrm{~B}-\mathrm{C} 45$ & $8.3(7)$ \\
\hline $\mathrm{C} 44-\mathrm{C} 44 \mathrm{~B}-\mathrm{C} 45 \mathrm{~B}-\mathrm{C} 46$ & $37.7(10)$ \\
\hline $\mathrm{C} 44-\mathrm{C} 44 \mathrm{~B}-\mathrm{C} 45 \mathrm{~B}-\mathrm{C} 46 \mathrm{~B}$ & $-93.7(9)$ \\
\hline $\mathrm{C} 44-\mathrm{C} 44 \mathrm{~B}-\mathrm{C} 45 \mathrm{~B}-\mathrm{C} 47 \mathrm{~B}$ & $95.3(14)$ \\
\hline $\mathrm{C} 44-\mathrm{C} 45 \mathrm{~B}-\mathrm{C} 47 \mathrm{~B}-\mathrm{C} 46$ & $-45.3(9)$ \\
\hline
\end{tabular}




\begin{tabular}{|c|c|c|c|}
\hline $\mathrm{O} 3 \mathrm{~A}-\mathrm{C} 10 \mathrm{~A}-\mathrm{C} 17 \mathrm{~A}-\mathrm{C} 18 \mathrm{~A}$ & $-6.6(3)$ & $\mathrm{C} 45-\mathrm{C} 44-\mathrm{C} 43 \mathrm{~B}-\mathrm{C} 42$ & $-177.2(6)$ \\
\hline $\mathrm{O} 5 \mathrm{~A}-\mathrm{C} 14 \mathrm{~A}-\mathrm{C} 16 \mathrm{~A}-\mathrm{O} 6 \mathrm{~A}$ & $1.8(3)$ & $\mathrm{C} 45-\mathrm{C} 44-\mathrm{C} 43 \mathrm{~B}-\mathrm{C} 43$ & $-126.5(7)$ \\
\hline $\mathrm{O} 5 \mathrm{~A}-\mathrm{C} 14 \mathrm{~A}-\mathrm{C} 16 \mathrm{~A}-\mathrm{C} 17 \mathrm{~A}$ & $177.3(2)$ & $\mathrm{C} 45-\mathrm{C} 44-\mathrm{C} 43 \mathrm{~B}-\mathrm{C} 42 \mathrm{~B}$ & $175.4(7)$ \\
\hline $\mathrm{O} 6 \mathrm{~A}-\mathrm{C} 16 \mathrm{~A}-\mathrm{C} 17 \mathrm{~A}-\mathrm{C} 10 \mathrm{~A}$ & $179.3(2)$ & $\mathrm{C} 45-\mathrm{C} 44-\mathrm{C} 43 \mathrm{~B}-\mathrm{C} 44 \mathrm{~B}$ & $-93.3(7)$ \\
\hline $\mathrm{O} 6 \mathrm{~A}-\mathrm{C} 16 \mathrm{~A}-\mathrm{C} 17 \mathrm{~A}-\mathrm{C} 18 \mathrm{~A}$ & $4.0(4)$ & $\mathrm{C} 45-\mathrm{C} 44-\mathrm{C} 44 \mathrm{~B}-\mathrm{C} 43$ & $160.6(13)$ \\
\hline $\mathrm{C} 1 \mathrm{~A}-\mathrm{O} 1 \mathrm{~A}-\mathrm{C} 2 \mathrm{~A}-\mathrm{C} 3 \mathrm{~A}$ & $-8.7(3)$ & $\mathrm{C} 45-\mathrm{C} 44-\mathrm{C} 44 \mathrm{~B}-\mathrm{C} 43 \mathrm{~B}$ & $106.8(8)$ \\
\hline $\mathrm{C} 1 \mathrm{~A}-\mathrm{O} 1 \mathrm{~A}-\mathrm{C} 2 \mathrm{~A}-\mathrm{C} 7 \mathrm{~A}$ & $172.2(3)$ & $\mathrm{C} 45-\mathrm{C} 44-\mathrm{C} 44 \mathrm{~B}-\mathrm{C} 45 \mathrm{~B}$ & $-11.9(9)$ \\
\hline $\mathrm{C} 1 \mathrm{~A}-\mathrm{O} 2 \mathrm{~A}-\mathrm{C} 3 \mathrm{~A}-\mathrm{C} 2 \mathrm{~A}$ & $9.3(3)$ & $\mathrm{C} 45-\mathrm{C} 46-\mathrm{C} 45 \mathrm{~B}-\mathrm{C} 43$ & $-43.2(7)$ \\
\hline $\mathrm{C} 1 \mathrm{~A}-\mathrm{O} 2 \mathrm{~A}-\mathrm{C} 3 \mathrm{~A}-\mathrm{C} 4 \mathrm{~A}$ & $-171.3(3)$ & $\mathrm{C} 45-\mathrm{C} 46-\mathrm{C} 45 \mathrm{~B}-\mathrm{C} 44$ & $-15.8(5)$ \\
\hline $\mathrm{C} 2 \mathrm{~A}-\mathrm{O} 1 \mathrm{~A}-\mathrm{C} 1 \mathrm{~A}-\mathrm{O} 2 \mathrm{~A}$ & $14.4(3)$ & $\mathrm{C} 45-\mathrm{C} 46-\mathrm{C} 45 \mathrm{~B}-\mathrm{C} 44 \mathrm{~B}$ & $-39.0(9)$ \\
\hline $\mathrm{C} 2 \mathrm{~A}-\mathrm{C} 3 \mathrm{~A}-\mathrm{C} 4 \mathrm{~A}-\mathrm{C} 5 \mathrm{~A}$ & $-1.4(4)$ & $\mathrm{C} 45-\mathrm{C} 46-\mathrm{C} 45 \mathrm{~B}-\mathrm{C} 46 \mathrm{~B}$ & $92.8(8)$ \\
\hline $\mathrm{C} 3 \mathrm{~A}-\mathrm{O} 2 \mathrm{~A}-\mathrm{C} 1 \mathrm{~A}-\mathrm{O} 1 \mathrm{~A}$ & $-14.6(3)$ & $\mathrm{C} 45-\mathrm{C} 46-\mathrm{C} 45 \mathrm{~B}-\mathrm{C} 47 \mathrm{~B}$ & $-159.6(9)$ \\
\hline $\mathrm{C} 3 \mathrm{~A}-\mathrm{C} 2 \mathrm{~A}-\mathrm{C} 7 \mathrm{~A}-\mathrm{C} 6 \mathrm{~A}$ & $2.1(4)$ & $\mathrm{C} 45-\mathrm{C} 46-\mathrm{C} 47 \mathrm{~B}-\mathrm{C} 45 \mathrm{~B}$ & $31.4(13)$ \\
\hline $\mathrm{C} 3 \mathrm{~A}-\mathrm{C} 4 \mathrm{~A}-\mathrm{C} 5 \mathrm{~A}-\mathrm{C} 6 \mathrm{~A}$ & $1.8(4)$ & $\mathrm{C} 45-\mathrm{C} 47-\mathrm{C} 48-\mathrm{O} 11$ & $94(7)$ \\
\hline $\mathrm{C} 4 \mathrm{~A}-\mathrm{C} 5 \mathrm{~A}-\mathrm{C} 6 \mathrm{~A}-\mathrm{C} 7 \mathrm{~A}$ & $-0.4(4)$ & $\mathrm{C} 45-\mathrm{C} 47-\mathrm{C} 48-\mathrm{C} 49$ & $-106(6)$ \\
\hline $\mathrm{C} 4 \mathrm{~A}-\mathrm{C} 5 \mathrm{~A}-\mathrm{C} 6 \mathrm{~A}-\mathrm{C} 8 \mathrm{~A}$ & $-178.8(2)$ & $\mathrm{C} 45-\mathrm{C} 45 \mathrm{~B}-\mathrm{C} 47 \mathrm{~B}-\mathrm{C} 46$ & $-15.6(7)$ \\
\hline $\mathrm{C} 5 \mathrm{~A}-\mathrm{C} 6 \mathrm{~A}-\mathrm{C} 7 \mathrm{~A}-\mathrm{C} 2 \mathrm{~A}$ & $-1.6(4)$ & $\mathrm{C} 46-\mathrm{C} 45-\mathrm{C} 47-\mathrm{C} 48$ & $55(7)$ \\
\hline $\mathrm{C} 5 \mathrm{~A}-\mathrm{C} 6 \mathrm{~A}-\mathrm{C} 8 \mathrm{~A}-\mathrm{C} 9 \mathrm{~A}$ & $-39.7(4)$ & $\mathrm{C} 46-\mathrm{C} 45-\mathrm{C} 45 \mathrm{~B}-\mathrm{C} 43$ & $143.9(6)$ \\
\hline $\mathrm{C} 5 \mathrm{~A}-\mathrm{C} 6 \mathrm{~A}-\mathrm{C} 8 \mathrm{~A}-\mathrm{C} 12 \mathrm{~A}$ & $137.8(2)$ & $\mathrm{C} 46-\mathrm{C} 45-\mathrm{C} 45 \mathrm{~B}-\mathrm{C} 44$ & $155.1(8)$ \\
\hline $\mathrm{C} 6 \mathrm{~A}-\mathrm{C} 8 \mathrm{~A}-\mathrm{C} 9 \mathrm{~A}-\mathrm{O} 3 \mathrm{~A}$ & $175.7(2)$ & $\mathrm{C} 46-\mathrm{C} 45-\mathrm{C} 45 \mathrm{~B}-\mathrm{C} 44 \mathrm{~B}$ & $146.8(7)$ \\
\hline $\mathrm{C} 6 \mathrm{~A}-\mathrm{C} 8 \mathrm{~A}-\mathrm{C} 12 \mathrm{~A}-\mathrm{O} 4 \mathrm{~A}$ & $2.9(4)$ & $\mathrm{C} 46-\mathrm{C} 45-\mathrm{C} 45 \mathrm{~B}-\mathrm{C} 46 \mathrm{~B}$ & $-104.0(8)$ \\
\hline $\mathrm{C} 6 \mathrm{~A}-\mathrm{C} 8 \mathrm{~A}-\mathrm{C} 12 \mathrm{~A}-\mathrm{C} 11 \mathrm{~A}$ & $-175.8(2)$ & $\mathrm{C} 46-\mathrm{C} 45-\mathrm{C} 45 \mathrm{~B}-\mathrm{C} 47 \mathrm{~B}$ & $14.7(6)$ \\
\hline $\mathrm{C} 7 \mathrm{~A}-\mathrm{C} 2 \mathrm{~A}-\mathrm{C} 3 \mathrm{~A}-\mathrm{O} 2 \mathrm{~A}$ & $178.8(2)$ & $\mathrm{C} 47-\mathrm{C} 45-\mathrm{C} 46-\mathrm{C} 45 \mathrm{~B}$ & $-153.7(9)$ \\
\hline $\mathrm{C} 7 \mathrm{~A}-\mathrm{C} 2 \mathrm{~A}-\mathrm{C} 3 \mathrm{~A}-\mathrm{C} 4 \mathrm{~A}$ & $-0.6(4)$ & $\mathrm{C} 47-\mathrm{C} 45-\mathrm{C} 46-\mathrm{C} 47 \mathrm{~B}$ & $176.9(13)$ \\
\hline $\mathrm{C} 7 \mathrm{~A}-\mathrm{C} 6 \mathrm{~A}-\mathrm{C} 8 \mathrm{~A}-\mathrm{C} 9 \mathrm{~A}$ & $141.9(3)$ & $\mathrm{C} 47-\mathrm{C} 45-\mathrm{C} 45 \mathrm{~B}-\mathrm{C} 43$ & $-149.8(18)$ \\
\hline $\mathrm{C} 7 \mathrm{~A}-\mathrm{C} 6 \mathrm{~A}-\mathrm{C} 8 \mathrm{~A}-\mathrm{C} 12 \mathrm{~A}$ & $-40.6(3)$ & $\mathrm{C} 47-\mathrm{C} 45-\mathrm{C} 45 \mathrm{~B}-\mathrm{C} 44$ & $-139(2)$ \\
\hline $\mathrm{C} 8 \mathrm{~A}-\mathrm{C} 6 \mathrm{~A}-\mathrm{C} 7 \mathrm{~A}-\mathrm{C} 2 \mathrm{~A}$ & $176.9(2)$ & $\mathrm{C} 47-\mathrm{C} 45-\mathrm{C} 45 \mathrm{~B}-\mathrm{C} 46$ & $66.3(19)$ \\
\hline $\mathrm{C} 9 \mathrm{~A}-\mathrm{O} 3 \mathrm{~A}-\mathrm{C} 10 \mathrm{~A}-\mathrm{C} 11 \mathrm{~A}$ & $1.4(3)$ & $\mathrm{C} 47-\mathrm{C} 45-\mathrm{C} 45 \mathrm{~B}-\mathrm{C} 44 \mathrm{~B}$ & $-146.9(19)$ \\
\hline $\mathrm{C} 9 \mathrm{~A}-\mathrm{O} 3 \mathrm{~A}-\mathrm{C} 10 \mathrm{~A}-\mathrm{C} 17 \mathrm{~A}$ & $-178.6(2)$ & $\mathrm{C} 47-\mathrm{C} 45-\mathrm{C} 45 \mathrm{~B}-\mathrm{C} 46 \mathrm{~B}$ & $-38(2)$ \\
\hline $\mathrm{C} 9 \mathrm{~A}-\mathrm{C} 8 \mathrm{~A}-\mathrm{C} 12 \mathrm{~A}-\mathrm{O} 4 \mathrm{~A}$ & $-179.5(2)$ & $\mathrm{C} 47-\mathrm{C} 45-\mathrm{C} 45 \mathrm{~B}-\mathrm{C} 47 \mathrm{~B}$ & $81(2)$ \\
\hline $\mathrm{C} 9 \mathrm{~A}-\mathrm{C} 8 \mathrm{~A}-\mathrm{C} 12 \mathrm{~A}-\mathrm{C} 11 \mathrm{~A}$ & $1.8(3)$ & $\mathrm{C} 42 \mathrm{~A}-\mathrm{C} 43 \mathrm{~A}-\mathrm{C} 44 \mathrm{~A}-\mathrm{C} 45 \mathrm{~A}$ & $-113(2)$ \\
\hline $\mathrm{C} 10 \mathrm{~A}-\mathrm{O} 3 \mathrm{~A}-\mathrm{C} 9 \mathrm{~A}-\mathrm{C} 8 \mathrm{~A}$ & $0.3(4)$ & $\mathrm{C} 43 \mathrm{~A}-\mathrm{C} 44 \mathrm{~A}-\mathrm{C} 45 \mathrm{~A}-\mathrm{C} 46 \mathrm{~A}$ & $-13(3)$ \\
\hline $\mathrm{C} 10 \mathrm{~A}-\mathrm{C} 11 \mathrm{~A}-\mathrm{C} 12 \mathrm{~A}-\mathrm{O} 4 \mathrm{~A}$ & $-178.9(2)$ & $\mathrm{C} 43 \mathrm{~A}-\mathrm{C} 44 \mathrm{~A}-\mathrm{C} 45 \mathrm{~A}-\mathrm{C} 47 \mathrm{~A}$ & $175(2)$ \\
\hline $\mathrm{C} 10 \mathrm{~A}-\mathrm{C} 11 \mathrm{~A}-\mathrm{C} 12 \mathrm{~A}-\mathrm{C} 8 \mathrm{~A}$ & $-0.2(3)$ & $\mathrm{C} 42 \mathrm{~B}-\mathrm{C} 40-\mathrm{C} 42-\mathrm{C} 43$ & $147(4)$ \\
\hline $\mathrm{C} 10 \mathrm{~A}-\mathrm{C} 11 \mathrm{~A}-\mathrm{C} 13 \mathrm{~A}-\mathrm{C} 14 \mathrm{~A}$ & $2.2(4)$ & $\mathrm{C} 42 \mathrm{~B}-\mathrm{C} 40-\mathrm{C} 42-\mathrm{C} 43 \mathrm{~B}$ & $95(4)$ \\
\hline $\mathrm{C} 10 \mathrm{~A}-\mathrm{C} 17 \mathrm{~A}-\mathrm{C} 18 \mathrm{~A}-\mathrm{C} 19 \mathrm{~A}$ & $171.8(2)$ & $\mathrm{C} 42 \mathrm{~B}-\mathrm{C} 43-\mathrm{C} 44-\mathrm{C} 45$ & $129.0(10)$ \\
\hline $\mathrm{C} 11 \mathrm{~A}-\mathrm{C} 10 \mathrm{~A}-\mathrm{C} 17 \mathrm{~A}-\mathrm{C} 16 \mathrm{~A}$ & $-1.6(4)$ & $\mathrm{C} 42 \mathrm{~B}-\mathrm{C} 43-\mathrm{C} 44-\mathrm{C} 43 \mathrm{~B}$ & $50.0(6)$ \\
\hline $\mathrm{C} 11 \mathrm{~A}-\mathrm{C} 10 \mathrm{~A}-\mathrm{C} 17 \mathrm{~A}-\mathrm{C} 18 \mathrm{~A}$ & $173.4(2)$ & $\mathrm{C} 42 \mathrm{~B}-\mathrm{C} 43-\mathrm{C} 44-\mathrm{C} 44 \mathrm{~B}$ & $154.1(16)$ \\
\hline $\mathrm{C} 11 \mathrm{~A}-\mathrm{C} 13 \mathrm{~A}-\mathrm{C} 14 \mathrm{~A}-\mathrm{O} 5 \mathrm{~A}$ & $179.4(2)$ & $\mathrm{C} 42 \mathrm{~B}-\mathrm{C} 43-\mathrm{C} 44-\mathrm{C} 45 \mathrm{~B}$ & $148.7(7)$ \\
\hline $\mathrm{C} 11 \mathrm{~A}-\mathrm{C} 13 \mathrm{~A}-\mathrm{C} 14 \mathrm{~A}-\mathrm{C} 16 \mathrm{~A}$ & $0.2(4)$ & $\mathrm{C} 42 \mathrm{~B}-\mathrm{C} 43-\mathrm{C} 43 \mathrm{~B}-\mathrm{C} 42$ & $0.6(3)$ \\
\hline $\mathrm{C} 12 \mathrm{~A}-\mathrm{C} 8 \mathrm{~A}-\mathrm{C} 9 \mathrm{~A}-\mathrm{O} 3 \mathrm{~A}$ & $-1.9(4)$ & $\mathrm{C} 42 \mathrm{~B}-\mathrm{C} 43-\mathrm{C} 43 \mathrm{~B}-\mathrm{C} 44$ & $-126.7(5)$ \\
\hline $\mathrm{C} 12 \mathrm{~A}-\mathrm{C} 11 \mathrm{~A}-\mathrm{C} 13 \mathrm{~A}-\mathrm{C} 14 \mathrm{~A}$ & $-178.0(2)$ & $\mathrm{C} 42 \mathrm{~B}-\mathrm{C} 43-\mathrm{C} 43 \mathrm{~B}-\mathrm{C} 44 \mathrm{~B}$ & $171.9(14)$ \\
\hline $\mathrm{C} 13 \mathrm{~A}-\mathrm{C} 11 \mathrm{~A}-\mathrm{C} 12 \mathrm{~A}-\mathrm{O} 4 \mathrm{~A}$ & $1.2(4)$ & $\mathrm{C} 42 \mathrm{~B}-\mathrm{C} 43-\mathrm{C} 44 \mathrm{~B}-\mathrm{C} 44$ & $-93(3)$ \\
\hline $\mathrm{C} 13 \mathrm{~A}-\mathrm{C} 11 \mathrm{~A}-\mathrm{C} 12 \mathrm{~A}-\mathrm{C} 8 \mathrm{~A}$ & $179.9(2)$ & $\mathrm{C} 42 \mathrm{~B}-\mathrm{C} 43-\mathrm{C} 44 \mathrm{~B}-\mathrm{C} 43 \mathrm{~B}$ & $-18(3)$ \\
\hline $\mathrm{C} 13 \mathrm{~A}-\mathrm{C} 14 \mathrm{~A}-\mathrm{C} 16 \mathrm{~A}-\mathrm{O} 6 \mathrm{~A}$ & $-178.9(2)$ & $\mathrm{C} 42 \mathrm{~B}-\mathrm{C} 43-\mathrm{C} 44 \mathrm{~B}-\mathrm{C} 45 \mathrm{~B}$ & $41(10)$ \\
\hline $\mathrm{C} 13 \mathrm{~A}-\mathrm{C} 14 \mathrm{~A}-\mathrm{C} 16 \mathrm{~A}-\mathrm{C} 17 \mathrm{~A}$ & $-3.4(4)$ & $\mathrm{C} 42 \mathrm{~B}-\mathrm{C} 43 \mathrm{~B}-\mathrm{C} 44 \mathrm{~B}-\mathrm{C} 43$ & $8.3(14)$ \\
\hline $\mathrm{C} 14 \mathrm{~A}-\mathrm{C} 16 \mathrm{~A}-\mathrm{C} 17 \mathrm{~A}-\mathrm{C} 10 \mathrm{~A}$ & $4.0(4)$ & $\mathrm{C} 42 \mathrm{~B}-\mathrm{C} 43 \mathrm{~B}-\mathrm{C} 44 \mathrm{~B}-\mathrm{C} 44$ & $105.9(8)$ \\
\hline $\mathrm{C} 14 \mathrm{~A}-\mathrm{C} 16 \mathrm{~A}-\mathrm{C} 17 \mathrm{~A}-\mathrm{C} 18 \mathrm{~A}$ & $-171.2(2)$ & $\mathrm{C} 42 \mathrm{~B}-\mathrm{C} 43 \mathrm{~B}-\mathrm{C} 44 \mathrm{~B}-\mathrm{C} 45 \mathrm{~B}$ & $-161.3(9)$ \\
\hline
\end{tabular}




\begin{tabular}{|c|c|c|c|}
\hline $\mathrm{C} 15 \mathrm{~A}-\mathrm{O} 5 \mathrm{~A}-\mathrm{C} 14 \mathrm{~A}-\mathrm{C} 13 \mathrm{~A}$ & $5.9(3)$ & $\mathrm{C} 43 \mathrm{~B}-\mathrm{C} 42-\mathrm{C} 43-\mathrm{C} 44$ & $-62.8(8)$ \\
\hline $\mathrm{C} 15 \mathrm{~A}-\mathrm{O} 5 \mathrm{~A}-\mathrm{C} 14 \mathrm{~A}-\mathrm{C} 16 \mathrm{~A}$ & $-174.9(2)$ & $\mathrm{C} 43 \mathrm{~B}-\mathrm{C} 42-\mathrm{C} 43-\mathrm{C} 42 \mathrm{~B}$ & $-6(4)$ \\
\hline $\mathrm{C} 16 \mathrm{~A}-\mathrm{O} 6 \mathrm{~A}-\mathrm{C} 20 \mathrm{~A}-\mathrm{C} 19 \mathrm{~A}$ & $-39.2(3)$ & $\mathrm{C} 43 \mathrm{~B}-\mathrm{C} 42-\mathrm{C} 43-\mathrm{C} 44 \mathrm{~B}$ & $28(4)$ \\
\hline $\mathrm{C} 16 \mathrm{~A}-\mathrm{O} 6 \mathrm{~A}-\mathrm{C} 20 \mathrm{~A}-\mathrm{C} 21 \mathrm{~A}$ & $-160.2(2)$ & $\mathrm{C} 43 \mathrm{~B}-\mathrm{C} 42-\mathrm{C} 43-\mathrm{C} 45 \mathrm{~B}$ & $36.9(12)$ \\
\hline $\mathrm{C} 16 \mathrm{~A}-\mathrm{O} 6 \mathrm{~A}-\mathrm{C} 20 \mathrm{~A}-\mathrm{C} 22 \mathrm{~A}$ & $80.6(2)$ & $\mathrm{C} 43 \mathrm{~B}-\mathrm{C} 43-\mathrm{C} 44-\mathrm{C} 45$ & $79.0(10)$ \\
\hline $\mathrm{C} 16 \mathrm{~A}-\mathrm{C} 17 \mathrm{~A}-\mathrm{C} 18 \mathrm{~A}-\mathrm{C} 19 \mathrm{~A}$ & $-13.3(4)$ & $\mathrm{C} 43 \mathrm{~B}-\mathrm{C} 43-\mathrm{C} 44-\mathrm{C} 44 \mathrm{~B}$ & $104.0(14)$ \\
\hline $\mathrm{C} 17 \mathrm{~A}-\mathrm{C} 10 \mathrm{~A}-\mathrm{C} 11 \mathrm{~A}-\mathrm{C} 12 \mathrm{~A}$ & $178.6(2)$ & $\mathrm{C} 43 \mathrm{~B}-\mathrm{C} 43-\mathrm{C} 44-\mathrm{C} 45 \mathrm{~B}$ & $98.7(6)$ \\
\hline $\mathrm{C} 17 \mathrm{~A}-\mathrm{C} 10 \mathrm{~A}-\mathrm{C} 11 \mathrm{~A}-\mathrm{C} 13 \mathrm{~A}$ & $-1.5(4)$ & $\mathrm{C} 43 \mathrm{~B}-\mathrm{C} 43-\mathrm{C} 42 \mathrm{~B}-\mathrm{C} 40$ & $-118.3(9)$ \\
\hline $\mathrm{C} 17 \mathrm{~A}-\mathrm{C} 18 \mathrm{~A}-\mathrm{C} 19 \mathrm{~A}-\mathrm{C} 20 \mathrm{~A}$ & $-5.6(4)$ & $\mathrm{C} 43 \mathrm{~B}-\mathrm{C} 43-\mathrm{C} 44 \mathrm{~B}-\mathrm{C} 44$ & $-75.2(10)$ \\
\hline $\mathrm{C} 18 \mathrm{~A}-\mathrm{C} 19 \mathrm{~A}-\mathrm{C} 20 \mathrm{~A}-\mathrm{O} 6 \mathrm{~A}$ & $30.6(3)$ & $\mathrm{C} 43 \mathrm{~B}-\mathrm{C} 43-\mathrm{C} 44 \mathrm{~B}-\mathrm{C} 45 \mathrm{~B}$ & $59(8)$ \\
\hline $\mathrm{C} 18 \mathrm{~A}-\mathrm{C} 19 \mathrm{~A}-\mathrm{C} 20 \mathrm{~A}-\mathrm{C} 21 \mathrm{~A}$ & $145.8(2)$ & $\mathrm{C} 43 \mathrm{~B}-\mathrm{C} 44-\mathrm{C} 45-\mathrm{C} 46$ & $43.4(11)$ \\
\hline $\mathrm{C} 18 \mathrm{~A}-\mathrm{C} 19 \mathrm{~A}-\mathrm{C} 20 \mathrm{~A}-\mathrm{C} 22 \mathrm{~A}$ & $-87.7(3)$ & $\mathrm{C} 43 \mathrm{~B}-\mathrm{C} 44-\mathrm{C} 45-\mathrm{C} 47$ & $-133.8(9)$ \\
\hline $\mathrm{C} 20 \mathrm{~A}-\mathrm{O} 6 \mathrm{~A}-\mathrm{C} 16 \mathrm{~A}-\mathrm{C} 14 \mathrm{~A}$ & $-160.6(2)$ & $\mathrm{C} 43 \mathrm{~B}-\mathrm{C} 44-\mathrm{C} 45-\mathrm{C} 45 \mathrm{~B}$ & $66.7(6)$ \\
\hline $\mathrm{C} 20 \mathrm{~A}-\mathrm{O} 6 \mathrm{~A}-\mathrm{C} 16 \mathrm{~A}-\mathrm{C} 17 \mathrm{~A}$ & $24.0(3)$ & $\mathrm{C} 43 \mathrm{~B}-\mathrm{C} 44-\mathrm{C} 44 \mathrm{~B}-\mathrm{C} 43$ & $53.8(11)$ \\
\hline $\mathrm{O} 7-\mathrm{C} 23-\mathrm{C} 24-\mathrm{C} 25$ & $179.8(2)$ & $\mathrm{C} 43 \mathrm{~B}-\mathrm{C} 44-\mathrm{C} 44 \mathrm{~B}-\mathrm{C} 45 \mathrm{~B}$ & $-118.6(7)$ \\
\hline $\mathrm{O} 7-\mathrm{C} 23-\mathrm{C} 28-\mathrm{C} 27$ & $-179.5(2)$ & $\mathrm{C} 43 \mathrm{~B}-\mathrm{C} 44 \mathrm{~B}-\mathrm{C} 45 \mathrm{~B}-\mathrm{C} 43$ & $54(7)$ \\
\hline $\mathrm{O} 8-\mathrm{C} 31-\mathrm{C} 32-\mathrm{C} 33$ & $1.1(4)$ & $\mathrm{C} 43 \mathrm{~B}-\mathrm{C} 44 \mathrm{~B}-\mathrm{C} 45 \mathrm{~B}-\mathrm{C} 44$ & $-80.2(10)$ \\
\hline $\mathrm{O} 8-\mathrm{C} 31-\mathrm{C} 32-\mathrm{C} 37$ & $-179.1(2)$ & $\mathrm{C} 43 \mathrm{~B}-\mathrm{C} 44 \mathrm{~B}-\mathrm{C} 45 \mathrm{~B}-\mathrm{C} 45$ & $-71.9(10)$ \\
\hline $\mathrm{O} 9-\mathrm{C} 33-\mathrm{C} 34-\mathrm{C} 35$ & $179.7(2)$ & $\mathrm{C} 43 \mathrm{~B}-\mathrm{C} 44 \mathrm{~B}-\mathrm{C} 45 \mathrm{~B}-\mathrm{C} 46$ & $-42.5(15)$ \\
\hline $\mathrm{C} 23-\mathrm{C} 24-\mathrm{C} 25-\mathrm{C} 26$ & $-0.5(4)$ & $\mathrm{C} 43 \mathrm{~B}-\mathrm{C} 44 \mathrm{~B}-\mathrm{C} 45 \mathrm{~B}-\mathrm{C} 46 \mathrm{~B}$ & $-173.8(9)$ \\
\hline $\mathrm{C} 24-\mathrm{C} 23-\mathrm{C} 28-\mathrm{C} 27$ & $0.7(4)$ & $\mathrm{C} 43 \mathrm{~B}-\mathrm{C} 44 \mathrm{~B}-\mathrm{C} 45 \mathrm{~B}-\mathrm{C} 47 \mathrm{~B}$ & $15.2(19)$ \\
\hline $\mathrm{C} 24-\mathrm{C} 25-\mathrm{C} 26-\mathrm{C} 27$ & $1.1(4)$ & $\mathrm{C} 44 \mathrm{~B}-\mathrm{C} 43-\mathrm{C} 44-\mathrm{C} 45$ & $-25.1(16)$ \\
\hline $\mathrm{C} 24-\mathrm{C} 25-\mathrm{C} 26-\mathrm{C} 29$ & $-177.2(2)$ & $\mathrm{C} 44 \mathrm{~B}-\mathrm{C} 43-\mathrm{C} 44-\mathrm{C} 43 \mathrm{~B}$ & $-104.0(14)$ \\
\hline $\mathrm{C} 25-\mathrm{C} 26-\mathrm{C} 27-\mathrm{C} 28$ & $-0.8(4)$ & $\mathrm{C} 44 \mathrm{~B}-\mathrm{C} 43-\mathrm{C} 44-\mathrm{C} 45 \mathrm{~B}$ & $-5.4(11)$ \\
\hline $\mathrm{C} 25-\mathrm{C} 26-\mathrm{C} 29-\mathrm{C} 30$ & $-178.9(2)$ & $\mathrm{C} 44 \mathrm{~B}-\mathrm{C} 43-\mathrm{C} 42 \mathrm{~B}-\mathrm{C} 40$ & $-98(3)$ \\
\hline $\mathrm{C} 26-\mathrm{C} 27-\mathrm{C} 28-\mathrm{C} 23$ & $-0.1(4)$ & $\mathrm{C} 44 \mathrm{~B}-\mathrm{C} 43-\mathrm{C} 42 \mathrm{~B}-\mathrm{C} 43 \mathrm{~B}$ & $21(3)$ \\
\hline $\mathrm{C} 26-\mathrm{C} 29-\mathrm{C} 30-\mathrm{C} 31$ & $-176.9(2)$ & $\mathrm{C} 44 \mathrm{~B}-\mathrm{C} 43-\mathrm{C} 43 \mathrm{~B}-\mathrm{C} 42$ & $-171.2(14)$ \\
\hline $\mathrm{C} 27-\mathrm{C} 26-\mathrm{C} 29-\mathrm{C} 30$ & $2.8(4)$ & $\mathrm{C} 44 \mathrm{~B}-\mathrm{C} 43-\mathrm{C} 43 \mathrm{~B}-\mathrm{C} 44$ & $61.5(12)$ \\
\hline $\mathrm{C} 28-\mathrm{C} 23-\mathrm{C} 24-\mathrm{C} 25$ & $-0.4(4)$ & $\mathrm{C} 44 \mathrm{~B}-\mathrm{C} 43-\mathrm{C} 43 \mathrm{~B}-\mathrm{C} 42 \mathrm{~B}$ & $-171.9(14)$ \\
\hline $\mathrm{C} 29-\mathrm{C} 26-\mathrm{C} 27-\mathrm{C} 28$ & $177.5(2)$ & $\mathrm{C} 44 \mathrm{~B}-\mathrm{C} 44-\mathrm{C} 45-\mathrm{C} 46$ & $-14.6(12)$ \\
\hline $\mathrm{C} 29-\mathrm{C} 30-\mathrm{C} 31-\mathrm{O} 8$ & $1.4(4)$ & $\mathrm{C} 44 \mathrm{~B}-\mathrm{C} 44-\mathrm{C} 45-\mathrm{C} 47$ & $168.1(9)$ \\
\hline $\mathrm{C} 29-\mathrm{C} 30-\mathrm{C} 31-\mathrm{C} 32$ & $-179.2(2)$ & $\mathrm{C} 44 \mathrm{~B}-\mathrm{C} 44-\mathrm{C} 45-\mathrm{C} 45 \mathrm{~B}$ & $8.6(7)$ \\
\hline $\mathrm{C} 30-\mathrm{C} 31-\mathrm{C} 32-\mathrm{C} 33$ & $-178.3(2)$ & $\mathrm{C} 44 \mathrm{~B}-\mathrm{C} 44-\mathrm{C} 43 \mathrm{~B}-\mathrm{C} 42$ & $-83.9(6)$ \\
\hline $\mathrm{C} 30-\mathrm{C} 31-\mathrm{C} 32-\mathrm{C} 37$ & $1.5(4)$ & $\mathrm{C} 44 \mathrm{~B}-\mathrm{C} 44-\mathrm{C} 43 \mathrm{~B}-\mathrm{C} 43$ & $-33.2(7)$ \\
\hline $\mathrm{C} 31-\mathrm{C} 32-\mathrm{C} 33-\mathrm{O} 9$ & $0.7(4)$ & $\mathrm{C} 44 \mathrm{~B}-\mathrm{C} 44-\mathrm{C} 43 \mathrm{~B}-\mathrm{C} 42 \mathrm{~B}$ & $-91.3(7)$ \\
\hline $\mathrm{C} 31-\mathrm{C} 32-\mathrm{C} 33-\mathrm{C} 34$ & $-179.9(2)$ & $\mathrm{C} 44 \mathrm{~B}-\mathrm{C} 45 \mathrm{~B}-\mathrm{C} 47 \mathrm{~B}-\mathrm{C} 46$ & $-96.0(13)$ \\
\hline $\mathrm{C} 31-\mathrm{C} 32-\mathrm{C} 37-\mathrm{C} 36$ & $-179.7(2)$ & $\mathrm{C} 45 \mathrm{~B}-\mathrm{C} 43-\mathrm{C} 44-\mathrm{C} 45$ & $-19.7(9)$ \\
\hline $\mathrm{C} 32-\mathrm{C} 33-\mathrm{C} 34-\mathrm{C} 35$ & $0.4(4)$ & $\mathrm{C} 45 \mathrm{~B}-\mathrm{C} 43-\mathrm{C} 44-\mathrm{C} 43 \mathrm{~B}$ & $-98.7(6)$ \\
\hline $\mathrm{C} 33-\mathrm{C} 32-\mathrm{C} 37-\mathrm{C} 36$ & $0.0(4)$ & $\mathrm{C} 45 \mathrm{~B}-\mathrm{C} 43-\mathrm{C} 44-\mathrm{C} 44 \mathrm{~B}$ & $5.4(11)$ \\
\hline $\mathrm{C} 33-\mathrm{C} 34-\mathrm{C} 35-\mathrm{C} 36$ & $-1.4(4)$ & $\mathrm{C} 45 \mathrm{~B}-\mathrm{C} 43-\mathrm{C} 42 \mathrm{~B}-\mathrm{C} 40$ & $-88.0(11)$ \\
\hline $\mathrm{C} 33-\mathrm{C} 34-\mathrm{C} 35-\mathrm{O} 10$ & $177.9(2)$ & $\mathrm{C} 45 \mathrm{~B}-\mathrm{C} 43-\mathrm{C} 42 \mathrm{~B}-\mathrm{C} 43 \mathrm{~B}$ & $30.3(10)$ \\
\hline $\mathrm{C} 34-\mathrm{C} 35-\mathrm{C} 36-\mathrm{C} 37$ & $1.7(4)$ & $\mathrm{C} 45 \mathrm{~B}-\mathrm{C} 43-\mathrm{C} 43 \mathrm{~B}-\mathrm{C} 42$ & $-165.2(5)$ \\
\hline $\mathrm{C} 34-\mathrm{C} 35-\mathrm{O} 10-\mathrm{C} 38$ & $-4.9(3)$ & $\mathrm{C} 45 \mathrm{~B}-\mathrm{C} 43-\mathrm{C} 43 \mathrm{~B}-\mathrm{C} 44$ & $67.5(4)$ \\
\hline $\mathrm{C} 35-\mathrm{C} 36-\mathrm{C} 37-\mathrm{C} 32$ & $-1.0(4)$ & $\mathrm{C} 45 \mathrm{~B}-\mathrm{C} 43-\mathrm{C} 43 \mathrm{~B}-\mathrm{C} 42 \mathrm{~B}$ & $-165.8(5)$ \\
\hline $\mathrm{C} 35-\mathrm{O} 10-\mathrm{C} 38-\mathrm{C} 39$ & $178.4(2)$ & $\mathrm{C} 45 \mathrm{~B}-\mathrm{C} 43-\mathrm{C} 43 \mathrm{~B}-\mathrm{C} 44 \mathrm{~B}$ & $6.0(12)$ \\
\hline $\mathrm{C} 36-\mathrm{C} 35-\mathrm{O} 10-\mathrm{C} 38$ & $174.3(2)$ & $\mathrm{C} 45 \mathrm{~B}-\mathrm{C} 43-\mathrm{C} 44 \mathrm{~B}-\mathrm{C} 44$ & $-134(8)$ \\
\hline $\mathrm{O} 10-\mathrm{C} 35-\mathrm{C} 36-\mathrm{C} 37$ & $-177.6(2)$ & $\mathrm{C} 45 \mathrm{~B}-\mathrm{C} 43-\mathrm{C} 44 \mathrm{~B}-\mathrm{C} 43 \mathrm{~B}$ & $-59(8)$ \\
\hline $\mathrm{O} 10-\mathrm{C} 38-\mathrm{C} 39-\mathrm{C} 40$ & $115.4(3)$ & $\mathrm{C} 45 \mathrm{~B}-\mathrm{C} 44-\mathrm{C} 45-\mathrm{C} 46$ & $-23.3(7)$ \\
\hline
\end{tabular}




$\begin{array}{ll}\mathrm{C} 37-\mathrm{C} 32-\mathrm{C} 33-\mathrm{O} 9 & -179.0(2) \\ \mathrm{C} 37-\mathrm{C} 32-\mathrm{C} 33-\mathrm{C} 34 & 0.3(4) \\ \mathrm{C} 38-\mathrm{C} 39-\mathrm{C} 40-\mathrm{C} 41 & 0.1(4) \\ \mathrm{C} 38-\mathrm{C} 39-\mathrm{C} 40-\mathrm{C} 42 & 172.5(4) \\ \mathrm{C} 38-\mathrm{C} 39-\mathrm{C} 40-\mathrm{C} 42 \mathrm{~A} & -177.2(5) \\ \mathrm{C} 38-\mathrm{C} 39-\mathrm{C} 40-\mathrm{C} 42 \mathrm{~B} & -177.2(5) \\ \mathrm{C} 39-\mathrm{C} 40-\mathrm{C} 42-\mathrm{C} 43 & -96.3(8) \\ \mathrm{C} 39-\mathrm{C} 40-\mathrm{C} 42-\mathrm{C} 43 \mathrm{~B} & -148.8(8) \\ \mathrm{C} 39-\mathrm{C} 40-\mathrm{C} 42 \mathrm{~A}-\mathrm{C} 43 \mathrm{~A} & -132.5(7) \\ \mathrm{C} 39-\mathrm{C} 40-\mathrm{C} 42 \mathrm{~B}-\mathrm{C} 43 & -95.2(5) \\ \mathrm{C} 39-\mathrm{C} 40-\mathrm{C} 42 \mathrm{~B}-\mathrm{C} 43 \mathrm{~B} & -137.4(7) \\ \mathrm{C} 40-\mathrm{C} 42-\mathrm{C} 43-\mathrm{C} 44 & -174.8(7) \\ \mathrm{C} 40-\mathrm{C} 42-\mathrm{C} 43-\mathrm{C} 42 \mathrm{~B} & -118(5) \\ \mathrm{C} 40-\mathrm{C} 42-\mathrm{C} 43-\mathrm{C} 43 \mathrm{~B} & -112.0(10) \\ \mathrm{C} 40-\mathrm{C} 42-\mathrm{C} 43-\mathrm{C} 44 \mathrm{~B} & -84(4) \\ \mathrm{C} 40-\mathrm{C} 42-\mathrm{C} 43-\mathrm{C} 45 \mathrm{~B} & -75.1(15)\end{array}$

$\begin{array}{ll}\mathrm{C} 45 \mathrm{~B}-\mathrm{C} 44-\mathrm{C} 45-\mathrm{C} 47 & 159.5(11) \\ \mathrm{C} 45 \mathrm{~B}-\mathrm{C} 44-\mathrm{C} 43 \mathrm{~B}-\mathrm{C} 42 & -119.8(6) \\ \mathrm{C} 45 \mathrm{~B}-\mathrm{C} 44-\mathrm{C} 43 \mathrm{~B}-\mathrm{C} 43 & -69.1(6) \\ \mathrm{C} 45 \mathrm{~B}-\mathrm{C} 44-\mathrm{C} 43 \mathrm{~B}-\mathrm{C} 42 \mathrm{~B} & -127.2(6) \\ \mathrm{C} 45 \mathrm{~B}-\mathrm{C} 44-\mathrm{C} 43 \mathrm{~B}-\mathrm{C} 44 \mathrm{~B} & -35.9(4) \\ \mathrm{C} 45 \mathrm{~B}-\mathrm{C} 44-\mathrm{C} 44 \mathrm{~B}-\mathrm{C} 43 & 172.4(15) \\ \mathrm{C} 45 \mathrm{~B}-\mathrm{C} 44-\mathrm{C} 44 \mathrm{~B}-\mathrm{C} 43 \mathrm{~B} & 118.6(7) \\ \mathrm{C} 45 \mathrm{~B}-\mathrm{C} 45-\mathrm{C} 46-\mathrm{C} 47 \mathrm{~B} & -29.4(12) \\ \mathrm{C} 45 \mathrm{~B}-\mathrm{C} 45-\mathrm{C} 47-\mathrm{C} 48 & 3(8) \\ \mathrm{C} 46 \mathrm{~B}-\mathrm{C} 45 \mathrm{~B}-\mathrm{C} 47 \mathrm{~B}-\mathrm{C} 46 & 93.4(10) \\ \mathrm{C} 47 \mathrm{~B}-\mathrm{C} 46-\mathrm{C} 45 \mathrm{~B}-\mathrm{C} 43 & 116.5(9) \\ \mathrm{C} 47 \mathrm{~B}-\mathrm{C} 46-\mathrm{C} 45 \mathrm{~B}-\mathrm{C} 44 & 143.8(8) \\ \mathrm{C} 47 \mathrm{~B}-\mathrm{C} 46-\mathrm{C} 45 \mathrm{~B}-\mathrm{C} 45 & 159.6(9) \\ \mathrm{C} 47 \mathrm{~B}-\mathrm{C} 46-\mathrm{C} 45 \mathrm{~B}-\mathrm{C} 44 \mathrm{~B} & 120.6(10) \\ \mathrm{C} 47 \mathrm{~B}-\mathrm{C} 46-\mathrm{C} 45 \mathrm{~B}-\mathrm{C} 46 \mathrm{~B} & -107.6(9) \\ & \end{array}$

